\title{
Simulações financeiras em GPU
}

\author{
Thársis Tuani Pinto Souza
}

\author{
DissertaÇÃo De Mestrado apresentada \\ $\mathrm{AO}$ \\ Instituto DE MatemáticA E Estatística \\ DA \\ Universidade DE SÃo PAUlo \\ PARA \\ OBTENÇÃO DO TÍTULO \\ DE \\ Mestre em CiÊnCIAS \\ Programa: Ciência da Computação \\ Orientador: Prof. Dr. Walter F. Mascarenhas
}

São Paulo, abril de 2013 


\section{Simulações financeiras em GPU}

Esta dissertação contém as correções e alterações sugeridas pela Comissão Julgadora durante a defesa realizada por Thársis Tuani Pinto Souza em 26/04/2013.

O original encontra-se disponível no Instituto de Matemática e Estatística da Universidade de São Paulo.

Comissão Julgadora:

- Prof. Dr. Walter F. Mascarenhas - IME-USP

- Prof. Dr. Herbert Kimura - Universidade de Brasília

- Prof. Dr. Marco Dimas Gubitoso - IME-USP 


\section{Resumo}

SOUZA, T. T. P. Simulações financeiras em GPU. Dissertação (Mestrado) - Instituto de Matemática e Estatística, Universidade de São Paulo, São Paulo, 2013.

É muito comum modelar problemas em finanças com processos estocásticos, dada a incerteza de suas variáveis de análise. Além disso, problemas reais nesse domínio são, em geral, de grande custo computacional, o que sugere a utilização de plataformas de alto desempenho (HPC) em sua implementação. As novas gerações de arquitetura de hardware gráfico (GPU) possibilitam a programação de propósito geral enquanto mantêm alta banda de memória e grande poder computacional. Assim, esse tipo de arquitetura vem se mostrando como uma excelente alternativa em HPC.

Com isso, a proposta principal desse trabalho é estudar o ferramental matemático e computacional necessário para modelagem estocástica em finanças com a utilização de GPUs como plataforma de aceleração. Para isso, apresentamos a GPU como uma plataforma de computação de propósito geral. Em seguida, analisamos uma variedade de geradores de números aleatórios, tanto em arquitetura sequencial quanto paralela. Além disso, apresentamos os conceitos fundamentais de Cálculo Estocástico e de método de Monte Carlo para simulação estocástica em finanças.

Ao final, apresentamos dois estudos de casos de problemas em finanças: "Stops Ótimos" e "Cálculo de Risco de Mercado". No primeiro caso, resolvemos o problema de otimização de obtenção do ganho ótimo em uma estratégia de negociação de ações de "Stop Gain". A solução proposta é escalável e de paralelização inerente em GPU. Para o segundo caso, propomos um algoritmo paralelo para cálculo de risco de mercado, bem como técnicas para melhorar a solução obtida. Nos nossos experimentos, houve uma melhora de 4 vezes na qualidade da simulação estocástica e uma aceleração de mais de 50 vezes.

Palavras-chave: Métodos Matemáticos em Finanças, Finanças Quantitativas, Modelagem Matemática, Computação Paralela, GPU, GPGPU, Números Aleatórios, Simulação Estocástica, Simulação de Equações Diferencias Estocásticas, Stops, Risco de Mercado, VaR, Value-at-Risk, Precificação de Opções 


\section{Abstract}

SOUZA, T. T. P. Finance and Stochastic Simulation on GPU. Dissertation (M.Sc.) - Instituto de Matemática e Estatística, Universidade de São Paulo, São Paulo, 2013.

Given the uncertainty of their variables, it is common to model financial problems with stochastic processes. Furthermore, real problems in this area have a high computational cost. This suggests the use of High Performance Computing (HPC) to handle them. New generations of graphics hardware (GPU) enable general purpose computing while maintaining high memory bandwidth and large computing power. Therefore, this type of architecture is an excellent alternative in HPC and comptutational finance.

The main purpose of this work is to study the computational and mathematical tools needed for stochastic modeling in finance using GPUs. We present GPUs as a platform for general purpose computing. We then analyze a variety of random number generators, both in sequential and parallel architectures, and introduce the fundamental mathematical tools for Stochastic Calculus and Monte Carlo simulation.

With this background, we present two case studies in finance: "Optimal Trading Stops" and "Market Risk Management". In the first case, we solve the problem of obtaining the optimal gain on a stock trading strategy of "Stop Gain". The proposed solution is scalable and with inherent parallelism on GPU. For the second case, we propose a parallel algorithm to compute market risk, as well as techniques for improving the quality of the solutions. In our experiments, there was a 4 times improvement in the quality of stochastic simulation and an acceleration of over 50 times.

Keywords: Mathematical Methods in Finance, Quantitative Finance, Mathematical Modeling, Parallel Computing, GPU, GPGPU, Random Numbers, Stochastic Simulation, Simulation of Stochastic Differential Equations, Stops, Market Risk, VaR, Value-at-Risk, Options Pricing 


\section{Agradecimentos}

Em primeiro lugar, à minha amada família: pai, Isaias de Souza Neto; mãe, Maria de Lourdes Pinto de Lacerda Souza; irmão, Heli Samuel Pinto Souza.

Também devo lembrar de inúmeros professores que foram fundamentais na minha formação intelectual. Ao Prof. Dr. Carlile Campos Lavor da Unicamp, responsável pela minha iniciação científica e que me deixou apenas lembranças de sabedoria e gentileza. Ao Prof. Dr. Orlando Stanley Juriaans da USP, que me motivou a lecionar com sua paixão pelo ensino e educação. À Profa. Dra. Yoshiko Wakabayashi, por ter me recebido no IME-USP em meu primeiro ano, sempre cordial e brilhante. Aos professores doutores Marco Dimas Gubitoso, Saulo Rabello Maciel de Barros e Marcel Jackowski pelas críticas em meu exame de qualificação de mestrado e pelas ricas discussões em inúmeros seminários em nosso grupo de estudo. Finalmente, ao Prof. Dr. Walter Mascarenhas, por ter aceito o desafio de me orientar e pelos ensinamentos em tantas áreas de conhecimento.

Não poderia também deixar de agradecer àqueles colegas de trabalho que me motivaram a completar o mestrado acadêmico: Gilberto Burgert, Sandro M. Manteiga e Juan Carlos Ruilova Terán.

Finalmente, agradeço aos amigos e colegas pelas revisões de rascunhos e sugestões: Tiago Montanher, Sandro M. Manteiga, André Valloto Lima, Filipe Polizel e Thiago Winkler 


\section{Sumário}

Lista de Abreviaturas $\quad$ xi

Lista de Símbolos e Notação $\quad$ xiii

Lista de Figuras $\quad$ xv

Lista de Tabelas $\quad$ xix

Lista de Algoritmos $\quad$ xxi

$\begin{array}{lll}\text { I Introdução } & 1\end{array}$

1 Introdução $\quad 3$

1.1 Aplicações . . . . . . . . . . . . . . . . . . . . . . 3

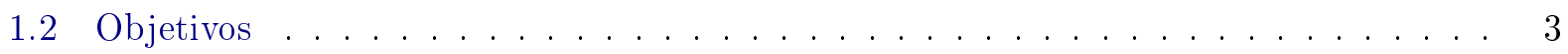

1.3 Organização do Trabalho . . . . . . . . . . . . . . . . . 5

$\begin{array}{lll}\text { II } & \text { Arquitetura Computacional } & 7\end{array}$

2 Modelos de Computação Paralela $\quad 9$

2.1 Classificação de Computadores Paralelos . . . . . . . . . . . . . . . . . . . 9

2.2 Arquiteturas de Memória em Computadores Paralelos . . . . . . . . . . . . . . . . . 10

2.2.1 Memória Compartilhada . . . . . . . . . . . . . . . . . . . . . 10

2.2 .2 Memória Distribuída . . . . . . . . . . . . . . . . . . . . . . 11

2.2 .3 Sistemas Híbridos . . . . . . . . . . . . . . . . . . . . . . 12

2.3 Métricas de Desempenho . . . . . . . . . . . . . . . . . . . . . 12

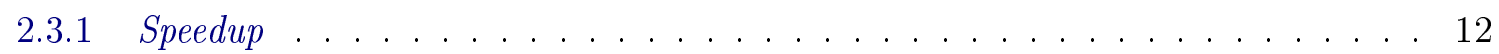

2.3 .2 Eficiência . . . . . . . . . . . . . . . . . . 13

2.3 .3 Escalabilidade . . . . . . . . . . . . . . . . . . 13

2.3.4 Taxa Sustentada de FLOPS . . . . . . . . . . . . . . . . . . . . . 14

3 Computação em GPU $\quad 15$

3.1 Arquitetura de GPU . . . . . . . . . . . . . . . . . . 16

3.1 .1 Breve Evolução Histórica . . . . . . . . . . . . . . . . . . . 16

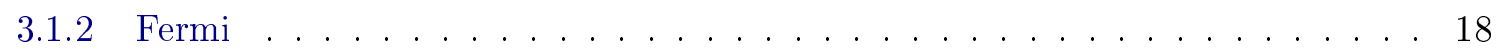




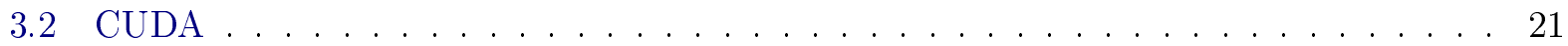

3.2.1 Modelo de Programação . . . . . . . . . . . . . . . . . . . 21

3.2.2 Modelo de Memória ........................... 23

4 Técnicas de Otimização de Desempenho em CUDA 25

4.1 Medição Tempo em CUDA . . . . . . . . . . . . . . . . . . . 25

4.2 Execução Concorrente Assíncrona . . . . . . . . . . . . . . . . . . . . . . 26

4.2.1 Comunicação Host-Device . . . . . . . . . . . . . . . . . . . . . . . . . . . . . 26

4.2.2 Kernels Paralelos ............................. 27

4.3 Otimizações de Memória . . . . . . . . . . . . . . . . . . . . . . . . 29

4.3.1 Coalesced Memory ............................ 29

4.3.2 Memória Compartilhada . . . . . . . . . . . . . . . . . . . . 29

4.3.3 Registradores ........................... 29

4.3.4 Memória Constante ........................... . 29

4.3.5 Memória Local . . . . . . . . . . . . . . . . . . . . . . . . . 30

4.4 Controle de Fluxo Condicional . . . . . . . . . . . . . . . . . . . . . . . 30

4.5 Configuração de Execução . . . . . . . . . . . . . . . . . . . . . . . . . . 31

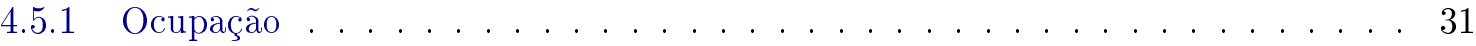

4.5.2 Configuração de Registradores . . . . . . . . . . . . . . . . . . . . . . . . . 32

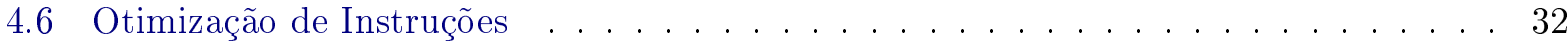

4.6.1 Bibliotecas Matemáticas . . . . . . . . . . . . . . . . . . . 32

4.6.2 Instruções Aritméticas . . . . . . . . . . . . . . . . . . . . . . . . 32

4.7 Sumário de Melhores Práticas . . . . . . . . . . . . . . . . . . . . . . . 32

5 Geração de Números Aleatórios em GPU 35

5.1 RNGs ................................ 35

5.1.1 Algoritmos Sequenciais de PRNGs . . . . . . . . . . . . . . . . . . . 36

5.1.2 Algoritmos Sequenciais de QRNGs ................... . . 37

5.1.3 Distribuições não Uniformes . . . . . . . . . . . . . . . . . . . . . . . 39

5.2 Técnicas de Paralelização de PRNGs . . . . . . . . . . . . . . . . . . . . . . . 41

5.2 .1 Central Server .......................... 41

5.2 .2 Sequence Splitting ............................ . . 41

5.2.3 Random Spacing ............................. 41

5.2 .4 Leap Frog . . . . . . . . . . . . . . . . . . . . . . . . . . . . . . 41

5.3 Multiple Recursive Generator MRG32k3a em GPU . . . . . . . . . . . . . . . . . . . 42

5.3.1 Formulação . . . . . . . . . . . . . . . . . . . . . . 42

5.3 .2 Paralelização . . . . . . . . . . . . . . . . . . . . . . . . 42

5.3.3 Implementação . . . . . . . . . . . . . . . . . . . . . . . . . 43

5.4 Sobol em GPU . . . . . . . . . . . . . . . . . . . . . . . . . . . . . . . . . . . . 43

5.4.1 Formulação . . . . . . . . . . . . . . . . . . . . . . . . . . 43

5.4 Paralelização ............................. 44

5.4 .3 Implementação . . . . . . . . . . . . . . . . . . . . . . . . . . . . 45

5.5 Bibliotecas para RNGs em GPU . . . . . . . . . . . . . . . . . . . . . . . 45

5.5.1 NVIDIA CURAND ......................... 45 
5.5 .2 Thrust::random . . . . . . . . . . . . . . . . . 45

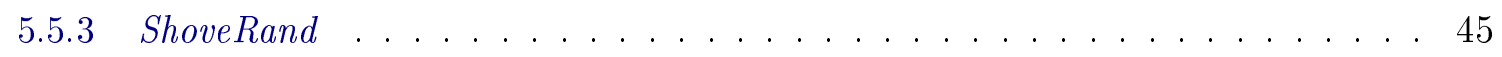

$\begin{array}{lll}\text { III } & \text { Fundamentos da Modelagem Matemática } & 47\end{array}$

6 Simulação Estocástica $\quad 49$

6.1 Fundamentos de Simulações de Monte Carlo . . . . . . . . . . . . . . . . . . . . . 49

6.1 .1 Integração de Monte Carlo . . . . . . . . . . . . . . . . . . . 49

6.1.2 Técnicas de Redução de Variância . . . . . . . . . . . . . . . . . . . . . . . . . . . . . . . . . . 50

6.2 Definições de Cálculo Estocástico . . . . . . . . . . . . . . . . . 53

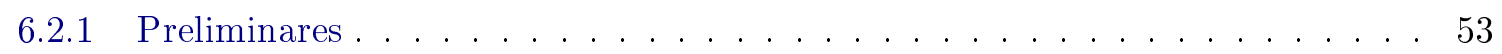

6.2 .2 Processos Estocásticos . . . . . . . . . . . . . . . 54

6.2 .3 Movimento Browniano . . . . . . . . . . . . . . . . . . 55

6.2 .4 Simulação do Movimento Browniano . . . . . . . . . . . . . . 56

6.3 Equações Diferenciais Estocásticas . . . . . . . . . . . . . . . 58

6.3 .1 Integral de Itồ . . . . . . . . . . . . . . . . . . 58

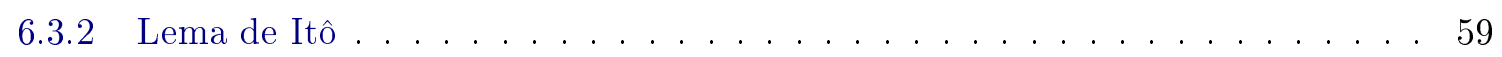

6.4 Soluções Numéricas de Equações Diferenciais Estocásticas . . . . . . . . . . . . . . 60

6.4 Equações Diferenciais Ordinárias . . . . . . . . . . . . . . . . . . . . . . . . . . 61

6.4 .2 Equações Diferenciais Estocásticas . . . . . . . . . . . . . . . . . . . 62

IV Estudo de Casos $\quad 65$

$\begin{array}{lll}7 & \text { Método } & 67\end{array}$

7.1 Ambiente Computacional . . . . . . . . . . . . . . . . 67

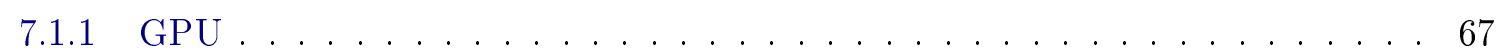

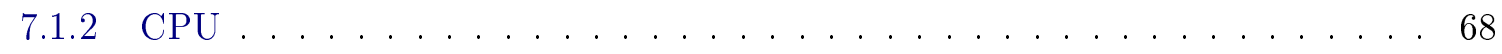

7.2 Tempo Computacional e Medidas . . . . . . . . . . . . . . . . . . 68

7.3 Dados Disponíveis . . . . . . . . . . . . . . . . . . . . 68

7.4 Geração de Números Aleatórios . . . . . . . . . . . . . . . . . . . 68

7.5 Configuração de Execução . . . . . . . . . . . . . . . . . . . 68

8 Stops Ótimos $\quad 71$

8.1 Formulação do Problema . . . . . . . . . . . . . . . . . . . . . . . 72

8.2 Modelagem em Tempo Discreto . . . . . . . . . . . . . . . . . 73

8.2.1 O Modelo Recursivo de (Warburtona e Zhang, 2006) . . . . . . . . . . . . . 73

8.2.2 Aproximação Binomial de (Cox et al., 1979) . . . . . . . . . . . . . . 75

8.2 .3 Simulação Estocástica do Modelo Trinomial . . . . . . . . . . . . . . . 76

8.2 .4 Notas sobre Stops Móveis . . . . . . . . . . . . . . . . . . 76

8.3 Modelagem Estocástica . . . . . . . . . . . . . . . . . . 77

8.3.1 Considerações Numéricas da Discretização de Euler . . . . . . . . . . . . . . . 79

8.4 Cálculo do Stop Ótimo . . . . . . . . . . . . . . . . . . . . 79

8.5 Análise Experimental e Discussão . . . . . . . . . . . . . . . . . . 80 
8.5.1 Experimentos do Modelo Trinomial . . . . . . . . . . . . . . . 80

8.5 .2 Experimentos do Modelo Estocástico . . . . . . . . . . . . . . . . . . 82

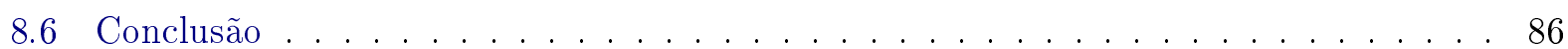

8.7 Notas e Leituras Complementares . . . . . . . . . . . . . . . . . . . . 86

9 Risco de Mercado $\quad 87$

9.1 Precificação de Opções . . . . . . . . . . . . . . . . . . . . . . . . . . . 87

9.1.1 O Método Tradicional de Black-Scholes . . . . . . . . . . . . . . . . . 88

9.1 .2 Precificação via Simulação . . . . . . . . . . . . . . . . . . . . . 90

9.2 Value-at-Risk . . . . . . . . . . . . . . . . . . . . . 92

9.2.1 Cálculo do VaR via Simulação de Monte Carlo . . . . . . . . . . . . . . . . . 94

9.2.2 Notas sobre Cálculo do VaR para Múltiplos Portfólios . . . . . . . . . . . . . 94

9.3 Análise Experimental e Discussão . . . . . . . . . . . . . . . . . . . . . . . 95

9.3.1 Experimentos da Precificação de Opções . . . . . . . . . . . . . . . . . 95

9.3.2 Experimentos do Cálculo de VaR . . . . . . . . . . . . . . . . . . 96

9.4 Conclusão . . . . . . . . . . . . . . . . . . . . . . . . . . 97

9.5 Notas e Leituras Complementares . . . . . . . . . . . . . . . . . . . . . . . 97

$\begin{array}{llr}\text { V Conclusão } & 101\end{array}$

10 Conclusão $\quad 103$

$\begin{array}{lll}\text { VI Apêndice } & 105\end{array}$

$\begin{array}{ll}\text { A NVIDIA CUDA } & 107\end{array}$

A.1 Compute Capability . . . . . . . . . . . . . . . . . 107

A.2 Funções Otimizadas pela Opção -use_fast_math . . . . . . . . . . . . . . . 108

$\begin{array}{ll}\text { Referências Bibliográficas } & 109\end{array}$

$\begin{array}{ll}\text { Índice Remissivo } & 115\end{array}$ 


\title{
Lista de Abreviaturas
}

\author{
API Interface de Programação de Aplicativos \\ (Application Programming Interface) \\ $\mathrm{Cg} \quad C$ for Graphics \\ CUDA Arquitetura de Dispositivo de Computação Unificada \\ (Computer Unified Device Architecture) \\ CMRG Combined Multiple Recursive Generator \\ CPU Unidade Central de Processamento \\ (Central Processing Unit) \\ DRAM Memória de Acesso Aleatório Dinâmico \\ (Dynamic Random-Access Memory) \\ ECC Código de Correção de Erro \\ (Error Correcting Code)
}

FLOPS Operações de Ponto Flutuante por Segundo

(Floating-Point Operations per Second)

FMA Multiplicação e Adição Fundidas

(Fused Multiply Add)

GDDR Graphic Double Data Rate

GPU Unidade de Processamento Gráfico

(Graphics Processing Unit)

GPGPU Computação de Propósito Geral em GPU

(General Purpose Computing on GPU)

HLSL High Level Shader Language

HPC Computação de Alto Desempenho

(High Performance Computing)

LCG Linear Congruential Generator

$\mathrm{LD} \backslash \mathrm{ST} \quad$ Carga $\backslash$ Armazenamento

(Load $\backslash$ Store)

LFS Linear Feedback Shift

MAD Multiplicação e Adição

MC Monte Carlo

MIMD Múltiplas Instruções e Múltiplos Dados

(Multiple Instructions Multiple Data)

MISD Múltiplas Instruções e Dado Único

(Multiple Instructions Single Data)

MRG Multiple Recursive Generator 
NUMA Acesso não Uniforme à Memória

(Nonuniform Memory Access)

PCIe Peripheral Component Interconnect Express

PL Processador Lógico

PRNG Gerador de Número Pseudo-aleatório (Pseudorandom Number Generator)

QRNG Gerador de Número Quasi-aleatório (Quasirandom Number Generator)

RNG Gerador de Número Aleatório (Random Number Generator)

SBD Sequências de Baixa Discrepância

SFU Unidade Especial de Função (Special Function Unit)

SIMD Instrução Única e Dados Múltiplos (Simple Instruction Multiple Data)

SISD Instrução Única e Dado Único (Single Instruction Single Data)

SMP Multiprocessadores Simétricos (Symmetric Multiprocessors)

SWC Substract With Carry

ULA Unidade Lógica e Aritmética

UPF Unidade de Ponto Flutuante

VGA Video Graphics Array 


\title{
Lista de Símbolos e Notação
}

\author{
$B, B_{t}, W_{t} \quad$ Movimento Browniano \\ $c_{X}(t, s), c_{X} \quad$ Função de covariância do processo $X$ \\ $\operatorname{cov}(X, Y) \quad$ Covariância das variáveis aleatórias $X$ e $Y$ \\ $\operatorname{correl}(X, Y)$ Correlação das variáveis aleatórias $X$ e $Y$ \\ $\mathbb{E}[X] \quad$ Esperança da variável aleatória $X$ \\ F $\quad$ Função de distribuição acumulada de uma variável aleatória \\ $\mu \quad$ Esperança de uma variável aleatória \\ $\mu_{X} \quad$ Esperança da variável aleatória $X$ \\ $\mathbb{N} \quad$ Conjunto dos números naturais \\ $\mathcal{N}\left(\mu, \sigma^{2}\right) \quad$ Distribuição normal com média $\mu$ e variância $\sigma$ \\ $\omega \quad \omega \in \Omega$, evento do espaço de eventos $\Omega$ \\ $\Omega \quad$ Espaço de eventos \\ $P \quad$ Medida de probabilidade \\ $\phi \quad$ Função da distribuição normal padrão \\ $\mathbb{R} \quad$ Linha real \\ $\mathbb{R}^{n} \quad$ Espaço Euclidiano $n$-dimensional \\ $\sigma \quad$ Desvio padrão \\ $\sigma_{X}^{2} \quad$ Variância da variável aleatória $X$ \\ $\sigma_{X}^{2}(t) \quad$ Função de variância do processo $X$ \\ $U(a, b) \quad$ Variável aleatória uniforme em $(a, b)$ \\ $\operatorname{var}(X) \quad$ Variância da variável aleatória $X$ \\ $X \quad$ Variável aleatória ou processo estocástico \\ $\bar{A} \quad$ Representa o complementar do conjunto $A$ \\ $\sim \quad$ Se $X \sim \mathcal{N}$, significa que a variável aleatória $X$ segue uma distribuição normal \\ $\stackrel{d}{=} \quad$ Se $X \stackrel{d}{=} Y$, significa que $X$ e $Y$ (variáveis aleatórias, vetores de variáveis aleatórias, \\ processos estocásticos) possuem a mesma distribuição de probabilidade \\ a.s. quase sempre \\ (almost surely)
}




\section{Lista de Figuras}

1.1 Estudo de caso GPU Tesla: Precificação de produtos - Bloomberg (NVIDIA, 2009b). 4

1.2 Estudo de caso GPU Tesla: Precificação de produtos - BNP-Paribas (NVIDIA, 2009b). 4

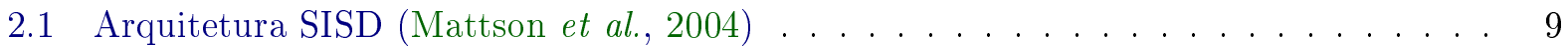

2.2 Arquitetura SIMD (Mattson et al., 2004) . . . . . . . . . . . . . . . . 10

2.3 Arquitetura MIMD (Mattson et al., 2004) . . . . . . . . . . . . . . . . . 10

2.4 Arquitetura SMP (Mattson et al., 2004) . . . . . . . . . . . . . . . 11

2.5 Arquitetura NUMA (Mattson et al., 2004) . . . . . . . . . . . . . . . . 11

2.6 Arquitetura de memória distribuída (Mattson et al., 2004) . . . . . . . . . . . . 11

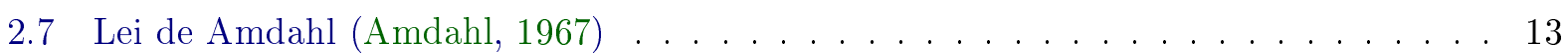

3.1 Poder computacional (GFLOP/s) CPU X GPU (Kirk e Hwu, 2010) . . . . . . . . . 15

3.2 Representação de Chip CPU X GPU (Kirk e Hwu, 2010) . . . . . . . . . . . . . . . . . . 16

3.3 Evolução Arquitetura GPU NVIDIA (Dally e Nickolls, 2010) . . . . . . . . . . . . . . 17

3.4 Evolução APIs para GPU (Brodtkorb et al., 2012) . . . . . . . . . . . . . . 17

3.5 Arquitetura Fermi (Dally e Nickolls, 2010) . . . . . . . . . . . . . . . . 18

3.6 Streaming Multiprocessor Fermi (Dally e Nickolls, 2010) . . . . . . . . . . . . . . 19

3.7 Instrução FMA (NVIDIA, 2009a) … . . . . . . . . . . . . . . . . 19

3.8 Exemplo de desempenho Fermi em aplicação de dupla precisão (NVIDIA, 2009a) . . 20

3.9 Agendador Fermi de warps (NVIDIA, 2009a) . . . . . . . . . . . . . . 20

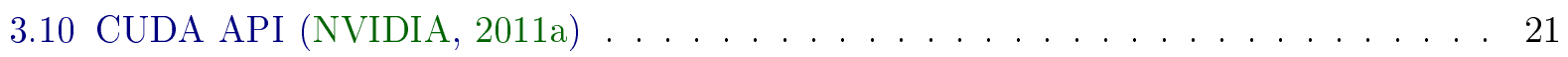

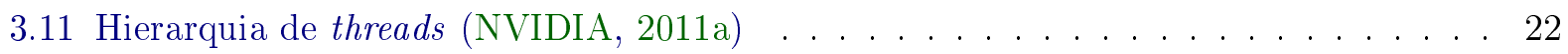

4.1 (I) Execução e cópia sequenciais; (II) Execução e cópia paralelos em compute capability 1.x; (III) Execução e cópia paralelos em compute capability 2.x; . . . . . . . . . 28

4.2 Acesso Coalesced Memory (NVIDIA, 2011a) . . . . . . . . . . . . . . . 29

4.3 Sobrecarga em conflitos de banco em memória compartilhada (Che et al., 2008). . . 30

4.4 Sobrecarga em threads divergentes (Che et al., 2008) . . . . . . . . . . . . 31

5.1 Sequência de Van Der Corput em base binária . . . . . . . . . . . . . . 38

5.2 Função Matlab para geração de números aleatórios uniformes (Huynh et al., 2011) . 39

5.3 Sequência de Halton bi-dimensional (Huynh et al., 2011) . . . . . . . . . . . . . . 39

5.4 ShoveRand meta-modelo (C. Mazel e Hill, 2011) . . . . . . . . . . . . . . . . 46 
6.1 Representação de um processo estocástico. Note que para $t=t_{1}$ temos que $X\left(t_{1}\right)$ é uma variável aleatória e que para cada evento $\omega_{i}$ é gerada uma trajetória correspondente.

7.1 NVIDIA GeForce GT $525 \mathrm{M} \ldots \ldots \ldots \ldots \ldots \ldots \ldots$

7.2 Arquitetura geral de execução de um estudo de caso. . . . . . . . . . . . . . . . . 69

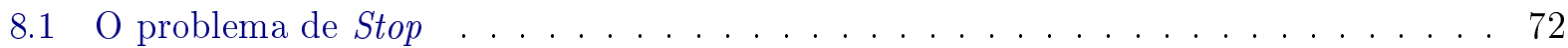

8.2 Exemplos de Tempo de Parada . . . . . . . . . . . . . . . . . . 73

8.3 Movimentos de preço possíveis para $T=7, K=3, L=-2 \ldots \ldots \ldots \ldots$. . . . 74

8.4 Transição no preço do ativo (Cox et al., 1979) . . . . . . . . . . . . . . . . 75

8.5 Probabilidade de Stop em função do preço de Stop Loss configurado. Valor do desvio padrão foi mantido fixo. . . . . . . . . . . . . . . . . . . . . . 81

8.6 Probabilidade de Stop em função do preço de Stop Loss configurado. Valor da média

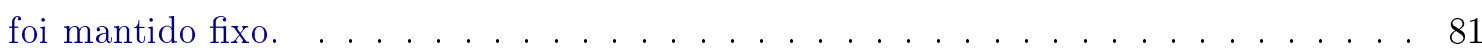

8.7 Probabilidade de Stop em função do preço de Stop Gain configurado. Valor do desvio padrão foi mantido fixo. . . . . . . . . . . . . . . . . . . . . . . 81

8.8 Probabilidade de Stop em função do preço de Stop Gain configurado. Valor da média foi mantido fixo.

8.9 Tempo gasto para cálculo de probabilidade de Stop Gain em função do preço de barreira configurado. . . . . . . . . . . . . . . . . . . 82

8.10 Simulação de passeios aleatórios do processo log-normal . . . . . . . . . . . . . . 83

8.11 Esperança de retorno de Stop Gain. Análise da variação da média $\mu$. . . . . . . . . 84

8.12 Esperança de retorno de Stop Gain. Análise da variação da volatilidade $\sigma$. . . . . . . 84

8.13 Esperança de retorno ótimo em função da taxa de juros. Configuração $\mu=0,0521139 \%$

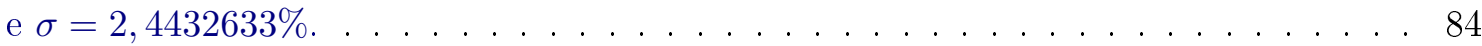

8.14 Tempo computacional gasto em cálculo da esperança de ganho de um Stop Gain em função do número de simulações. Configuração: Stop $=65, \mu=-0,00055 \%, \sigma=2 \% . \quad 85$

8.15 Tempo computacional gasto em cálculo da esperança de ganho de um Stop Gain. Configuração: número de simulações $=38.400, \mu=-0,00055 \%, \sigma=2 \%$. . . . . . 85

9.1 Contrato de Opção de Compra de Taxa de Câmbio de Reais por Dólar Comercial fornecido pela BM\&FBovespa . . . . . . . . . . . . . . . . . . 88

9.2 Payoffs das posições em opções europeias: (a) compra de Call; (b) venda de Call; (c) compra de Put; (d) venda de Put. Retirado de (Hull, 2012). . . . . . . . . . . . . . . 91

9.3 Resumo comparativo de metodologias de cálculo de VaR. Retirado de (Jorion, 2003) 93

9.4 Simulação do prêmio de uma opção de call europeia em diferentes RNGs. . . . . . . 96

9.5 Aplicação de técnicas de redução de variância na simulação do prêmio de uma opção de call europeia utilizando Sobol como RNG. . . . . . . . . . . . . . . . . . 96

9.6 Variação no valor do Portfólio de Opções. Número de Simulações fixo em 128. . . . . 98

9.7 Variação no valor do Portfólio de Opções. Número de Simulações fixo em 1.280. 98

9.8 Variação no valor do Portfólio de Opções. Número de Simulações fixo em 51.200. . 98

9.9 Speedup no cálculo de VaR de uma carteira com 2000 opções em função do número de simulações. . . . . . . . . . . . . . . . . . . . . . . . 99 
9.10 Speedup no cálculo de VaR em função do número de opções no portfólio. Número de simulações fixo em $51200 \ldots \ldots$. . . . . . . . . . . . . . 100

A.1 Especificação técnica por Compute Capability . . . . . . . . . . . . . . . . . 107

A.2 Funções afetadas por -use_fast_math . . . . . . . . . . . . . 108 


\section{Lista de Tabelas}

3.1 Sumário de Características Arquitetura Fermi . . . . . . . . . . . . . . . . . 21

3.2 Extensões de função em CUDA . . . . . . . . . . . . . . . . . . 23

3.3 Tipos de Memória . . . . . . . . . . . . . . . . . . . . . 23

4.1 Sumário de estratégias de otimização de desempenho . . . . . . . . . . . . . 33

5.1 Sequência de Halton . . . . . . . . . . . . . . . . . . 38

7.1 Características da placa gráfica NVIDIA GeForce GT 525M. . . . . . . . . . . . 67

9.1 Tempo Computacional gasto em CPU e GPU no cálculo de VaR em função do número de simulações. Número de opções fixo em 2.000 . . . . . . . . . . . . . . . . . 99

9.2 Tempo Computacional gasto em CPU e GPU no cálculo de VaR em função do número de opções no portfólio. Número de simulações fixo em 51200 . . . . . . . . . . . . 99 


\section{Lista de Algoritmos}

1 Sequência de Van Der Corput . . . . . . . . . . . . . . . 37

2 Simulação do Movimento Browniano . . . . . . . . . . . . . . . 57

3 Esperança de uma função de variável aleatória via discretização de Euler . . . . . . . 64

4 Gera $X \sim U_{3}(x)$, distribuição de probabilidade trinomial de preços . . . . . . . . 76

$5 \quad$ Kernel: Calcula Probabilidade de Stop Gain . . . . . . . . . . . . . . . 77

6 Kernel: Valor Esperado do Ganho de um Stop Gain K . . . . . . . . . . . . 79

7 Cálculo do valor esperado do prêmio de uma opção europeia de call via simulação utilizando técnicas de redução de variância. . . . . . . . . . . . . . . . . . . 92

8 Kernel: Cálculo do VaR pelo Método de Simulação de Monte Carlo . . . . . . . . . . 94 
Parte I

\section{Introdução}





\section{Capítulo 1}

\section{Introdução}

Avanços em computação e algoritmos estabelecem uma nova área interdisciplinar ao combinar finanças e ciência da computação. Com isso, a efetiva exploração de novos métodos computacionais tem ajudado as instituições financeiras em sua tomada de decisões e no seu gerenciamento de risco. Nesse contexto, é comum modelar problemas em finanças utilizando processos estocásticos, dada à incerteza de suas variáveis. Além disso, problemas reais nesse domínio são, em geral, de grande custo computacional. Isso sugere a utilização de plataformas de alto desempenho (HPC) em sua implementação. As novas gerações de arquitetura de GPU possibilitam a programação de propósito geral enquanto mantêm alta banda de memória e grande poder computacional. Assim, essa arquitetura é uma excelente alternativa em HPC. Em (Lee et al., 2010a), os autores estudam a viabilidade da utilização de placas gráficas em simulações estocásticas e concluem:

"We believe the speedup we observe should motivate wider use of parallelizable simulation methods and greater methodological attention to their design." 1

Assim, a proposta desse trabalho é estudar o ferramental matemático e computacional necessário para modelagem estocástica em finanças com a utilização de GPUs de propósito geral como plataforma de aceleração.

\section{$1.1 \quad$ Aplicações}

Com o recente advento de placas gráficas programáveis, sua aplicação tem sido realizada em diferentes domínios, como: otimização linear (Jung e O'Leary, 2009), (Spampinato e Elstery, 2009); resolução de equações diferenciais parciais (Egloff, 2010); algoritmos de ordenação (Satish et al., 2009); grafos (Buluc et al., 2010), (Dehne e Yogaratnam, 2010), (Harish e Narayanan, 2007); bioinformática (Stojanovski et al., 2012), (Sadiq et al., 2012) ou física (Muller e Frauendiener, 2013), (Saito et al., 2012).

Em finanças, podemos citar aplicações como: precificação de produtos (Solomon et al., 2010), (Pages e Wilbertz, 2010), (Pages e Wilbertz, 2012); cálculo de risco (Dixon et al., 2009) ou econofísica (Preis, 2011). Além disso, há grandes instituições financeiras com casos de sucesso ao migrar sua arquitetura de HPC para GPU. As figuras 1.1 e 1.2 apresentam alguns exemplos. Como podemos ver, em ambos os casos, as instituições obtiveram uma aceleração considerável, com redução de custo e economia de energia.

\subsection{Objetivos}

Há diferentes níveis de análise de simulações estocásticas financeiras em GPU. Em nível mais baixo, é importante estudar a plataforma computacional utilizada, o modelo de computação paralela, as características da arquitetura alvo e considerações de otimização de desempenho da mesma.

\footnotetext{
${ }^{1}$ Acreditamos que a aceleração que observamos deve motivar a maior utilização de métodos de simulação paralelizáveis e uma atenção metodológica maior para esse tipo de modelagem.
} 


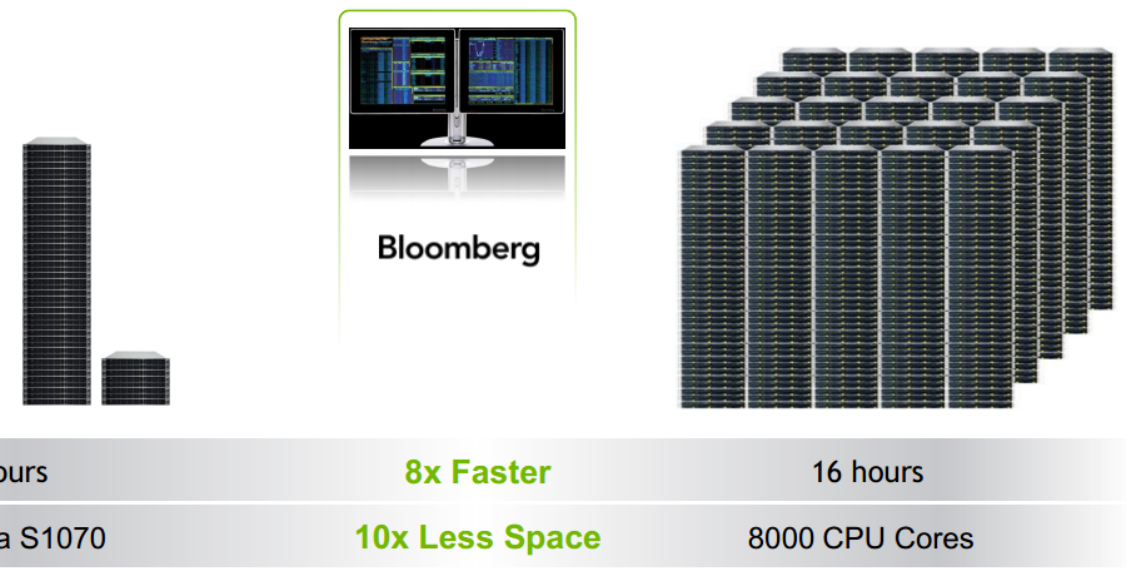

Figura 1.1: Estudo de caso GPU Tesla: Precificação de produtos - Bloomberg (NVIDIA, 2009b).

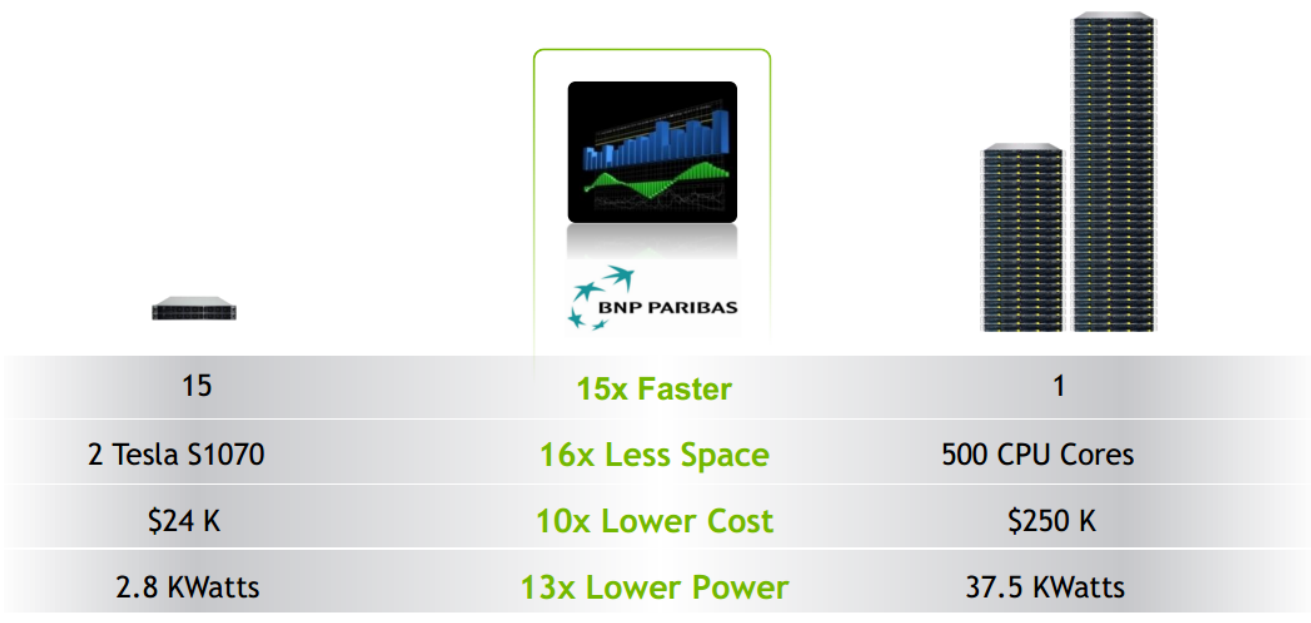

Figura 1.2: Estudo de caso GPU Tesla: Precificação de produtos - BNP-Paribas (NVIDIA, 2009b).

Em geral, simulações estocásticas são sensíveis à fonte de aleatoriedade que caracteriza a qualidade estatística de seus resultados. Dessa forma, também é de fundamental importância o estudo de geradores de números aleatórios e a portabilidade dos mesmos na arquitetura utilizada. Em nível mais alto, é necessário um ferramental matemático em processos estocásticos e simulação de Monte Carlo para resolução de grande parte dos problemas em finanças.

Este trabalho é um estudo em todos esses níveis. Ademais, apresentamos dois estudos de casos de problemas reais em finanças. Assim, os objetivos desse trabalho são os seguintes:

- Utilizar um modelo de hardware e programação para GPU, apresentando essa plataforma como um verdadeiro co-processador genérico.

- Estudo de geradores de números aleatórios sequenciais e paralelos.

- Apresentação de ferramental matemático fundamental para modelagem estocástica em finanças.

- Resolver problemas reais em finanças ao aplicar a teoria apresentada.

Na última parte do trabalho, apresentamos dois estudos de casos em finanças: "Stops Ótimos" e "Cálculo de Risco de Mercado". No primeiro problema, após uma revisão da literatura, propomos uma alternativa de resolução via simulação estocástica, em vista à escalabilidade da solução e portabilidade em GPU. Para o segundo problema, propomos um algoritmo em GPU com objetivo de aceleração significativa, bem como técnicas de melhoria da qualidade da solução obtida. 


\subsection{Organização do Trabalho}

Além da introdução, o presente trabalho é organizado em quatro partes e um apêndice. Na segunda parte, estudamos a arquitetura computacional de GPU. Para isso, apresentamos modelos tradicionais de computação paralela no Capítulo 2; realizamos uma introdução à computação de propósito geral em GPU no Capítulo 3; estudamos técnicas de otimização nessa arquitetura no Capítulo 4 e finalizamos com um estudo de geradores de números aleatórios e sua portabilidade para GPU. A terceira parte apresenta o ferramental básico para modelagem estocástica em finanças. Assim, na seção 6.1, discutimos fundamentos de Simulações de Monte Carlo. Em seguida, nas seções 6.2 e 6.3, abordamos processos estocásticos e resolução de Equações Diferenciais Estocásticas. Ao final, estudamos métodos práticos de solução numérica dessas equações, na seção 6.4. Na quarta parte descrevemos estudo de casos de dois problemas reais em finanças em uma plataforma de GPU. Na última parte, concluímos o trabalho. 


\section{Parte II}

Arquitetura Computacional 



\section{Capítulo 2}

\section{Modelos de Computação Paralela}

Computador paralelo é definido por (Almasi e Gottlieb, 1989) como uma coleção de elementos de processamento que cooperam e se comunicam para resolver grandes problemas de forma rápida. Nas próximas seções classificamos os principais tipos de computadores paralelos, abordamos suas arquiteturas de memória e, por fim, apresentamos maneiras de medir o seu desempenho.

\subsection{Classificação de Computadores Paralelos}

A taxonomia de Flynn (Flynn, 1972) é a maneira mais comum de se caracterizar arquiteturas de computadores paralelos. Segunda ela, há quatro categorias: SISD, SIMD, MISD e MIMD.

Em um sistema SISD (Single Instruction Single Data ${ }^{1}$ ), não há exploração de paralelismo. Nela cada instrução opera sobre um único fluxo de dados, como mostrado na figura 2.1. Essa classificação corresponde à arquitetura clássica de Von Neumann.

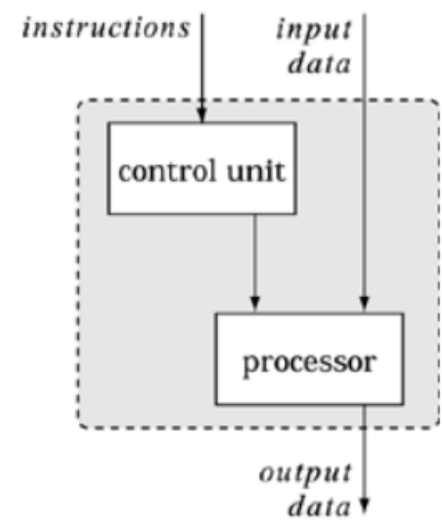

Figura 2.1: Arquitetura SISD (Mattson et al., 2004)

Arquiteturas SIMD (Single Instruction, Multiple Data ${ }^{2}$ ) são caracterizadas por um único fluxo de instruções que opera sobre múltiplos dados, como mostrado na figura 2.2. Essa arquitetura é ideal para aplicações que executam o mesmo conjunto de operações em um grande volume de dados. Arquiteturas de GPU se enquadram nessa classificação.

Arquiteturas MISD (Multiple Instruction, Single Data ${ }^{3}$ ) são conceitualmente definidas como de múltiplos fluxos de instrução que operam sobre os mesmos dados. Na prática, não é comum encontrar implementações desse modelo.

\footnotetext{
${ }^{1}$ em português: instrução única e dados únicos

${ }^{2}$ em português: instrução única e dados múltiplos

${ }^{3}$ em português: instruções múltiplas e dados únicos
} 


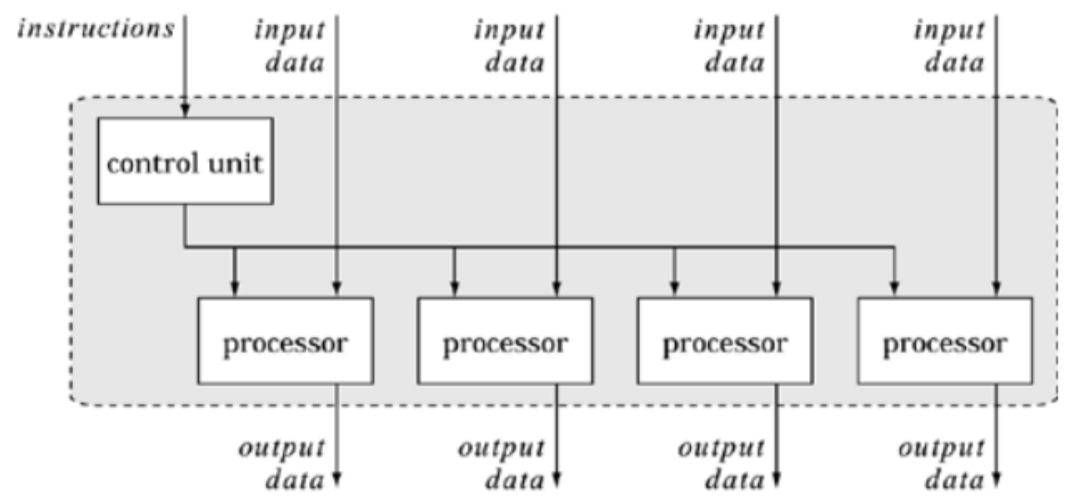

Figura 2.2: Arquitetura SIMD (Mattson et al., 2004)

Finalmente, a classificação MIMD (Multiple Instruction, Multiple Data ${ }^{4}$ ) diz respeito a unidades de processamento que executam múltiplas instruções ao mesmo tempo em diferentes conjuntos de dados. Como visto na figura 2.3, essa é a arquitetura mais genérica dentre as nomeadas por Flynn. $\mathrm{Na}$ verdade, todas as outras classificações são sub-casos da arquitetura MIMD.

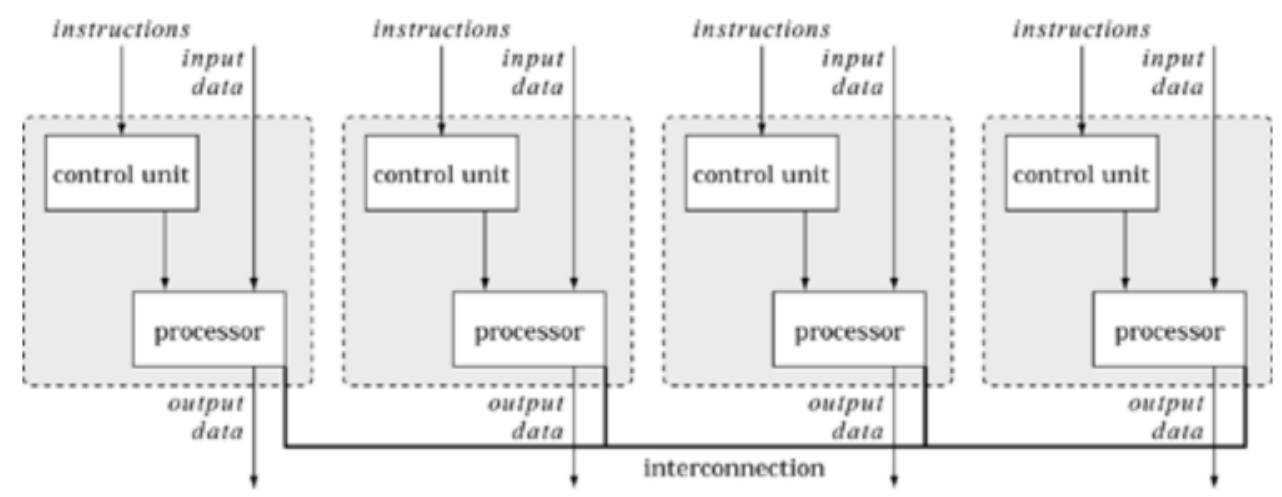

Figura 2.3: Arquitetura MIMD (Mattson et al., 2004)

\subsection{Arquiteturas de Memória em Computadores Paralelos}

A arquitetura MIMD da classificação de Flynn é muito genérica para ser utilizada na prática. Assim, ela é, geralmente, decomposta de acordo com a organização de memória.

\subsubsection{Memória Compartilhada}

Em um sistema de memória compartilhada, todos os processadores compartilham um mesmo espaço de endereçamento e se comunicam por meio de leitura e escrita em variáveis compartilhadas.

SMPs (symmetric multiprocessors ${ }^{5}$ ) são uma classe comum de sistemas de memória compartilhada. Como mostrado na figura 2.4, todos os processadores compartilham uma conexão com uma memória em comum e acessam todos os locais de memória em mesma velocidade. Nesses sistemas, em geral, não é necessário distribuir estruturas de dados ao longo dos múltiplos processadores, já que os mesmos acessam os dados de maneira compartilhada. Entretanto, como o canal de acesso aos dados é único, o aumento no número de processadores pode tornar a largura de banda em um

\footnotetext{
${ }^{4}$ em português: instruções múltiplas e dados múltiplos

${ }^{5}$ em português: multiprocessadores simétricos
} 
fator limitante.

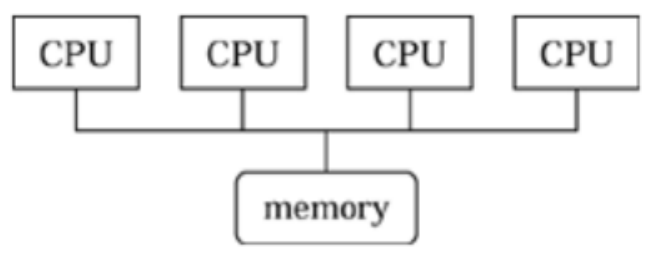

Figura 2.4: Arquitetura SMP (Mattson et al., 2004)

NUMA (nonuniform memory access ${ }^{6}$ ) é outra classe importante de sistemas de memória compartilhada. Como visto na figura 2.5, a memória é compartilhada. Contudo, alguns blocos de memória podem ficar fisicamente mais próximos com certos processadores e são naturalmente associados a eles. Isso pode reduzir o problema de largura de banda enunciado anteriormente em sistemas SMP e, portanto, pode permitir a utilização de um número maior de processadores.

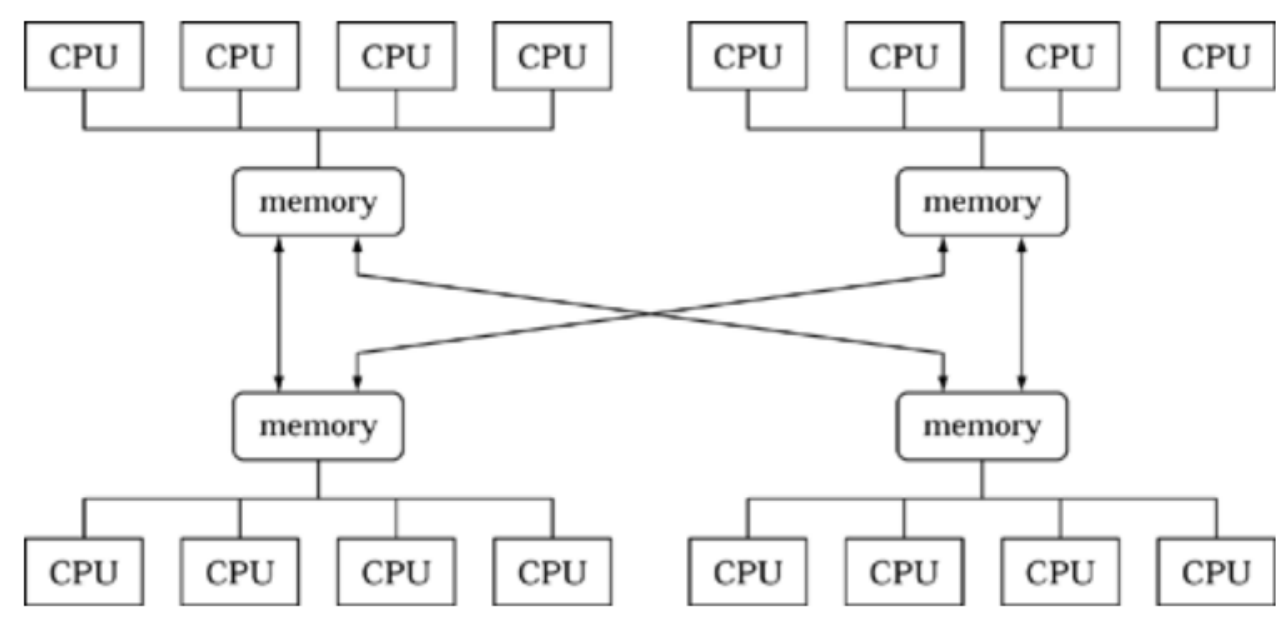

Figura 2.5: Arquitetura NUMA (Mattson et al., 2004)

\subsubsection{Memória Distribuída}

Em sistemas de memória distribuída, cada processo tem seu espaço de memória próprio e a comunicação é feita por meio de troca de mensagens ${ }^{7}$. A figura 2.6 apresenta um exemplo representativo de um computador de memória distribuída.

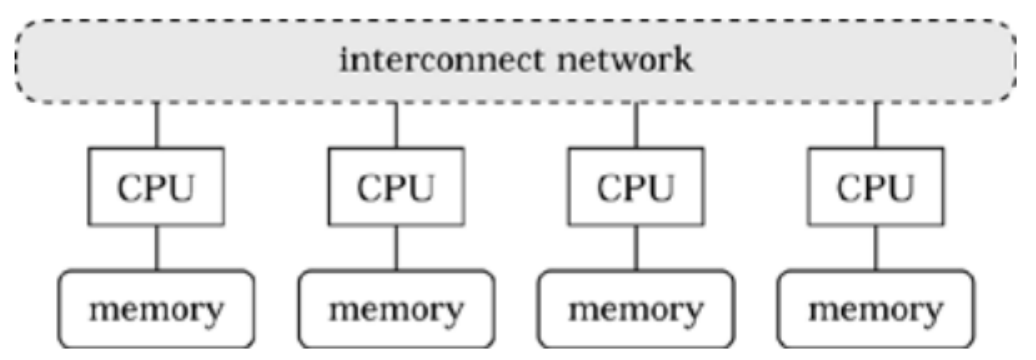

Figura 2.6: Arquitetura de memória distribuída (Mattson et al., 2004)

\footnotetext{
${ }^{6}$ em português: acesso não uniforme à memória

${ }^{7}$ no original: message passing
} 
Dependendo da topologia e da tecnologia utilizadas para interconexão dos processadores, a velocidade de comunicação pode ser tão rápida quanto em uma arquitetura de memória compartilhada (e.g. supercomputadores com alta integração) ou ser de reduzido desempenho (e.g. em um agregado de computadores interconectados via rede Ethernet). É importante observar que esta arquitetura requer a configuração explícita da comunicação entre os processadores e a preocupação na distribuição de dados aos mesmos.

\subsubsection{Sistemas Híbridos}

Como o nome sugere, sistemas híbridos são aqueles que utilizam ambas as formas de organização de memória aqui citadas. Esses sistemas, geralmente, correspondem a agregados de nós com espaços de endereçamento separados, onde cada nó contem múltiplos processadores que compartilham memória.

\subsection{Métricas de Desempenho}

Em uma arquitetura sequencial, o tempo de execução de um programa se dá em função do tamanho da entrada e do espaço (memória). Já em arquiteturas paralelas, além dessas grandezas, o número de processadores e parâmetros específicos de comunicação da arquitetura alvo influenciam o tempo de execução. Isso implica que é necessário analisar algoritmos paralelos tendo em vista a arquitetura alvo particular.

Nesse sentido, há algumas métricas de desempenho comumente utilizadas em sistemas paralelos como:

- Speedup

- Eficiência ${ }^{8}$

- Escalabilidade ${ }^{9}$

- Taxa sustentada de FLOPS ${ }^{10}$

\subsubsection{Speedup}

Razão entre o tempo de execução do algoritmo executado em um único processador e o tempo de execução do mesmo algoritmo em múltiplos processadores:

$$
S_{p}=\frac{T_{1}}{T_{p}}
$$

onde,

- $p$ é o número de processadores

- $T_{1}$ é o tempo de execução do algoritmo sequencial

- $T_{p}$ é o tempo do algoritmo paralelo em $p$ processadores

Em comparação com o número de processadores, o speedup pode ser classificado assim:

- $S_{p}=p$, Linear speedup

- $S_{p}<p$, Sub-linear speedup

\footnotetext{
${ }^{8}$ no original: efficiency

${ }^{9}$ no original: scalability

${ }^{10}$ no original: sustained FLOP rate
} 
- $S_{p}>p$, Super-linear speedup

A Lei de Amdahl descreve o máximo speedup $(S)$ esperado ao paralelizar uma certa porção de um programa sequencial:

$$
S=\frac{1}{(1-P)+\frac{P}{p}}
$$

onde $P$ é a fração do tempo gasto pelo programa serial da parte do código que pode ser paralelizado e $p$ é o número de processadores sobre o qual o código paralelizável é executado.

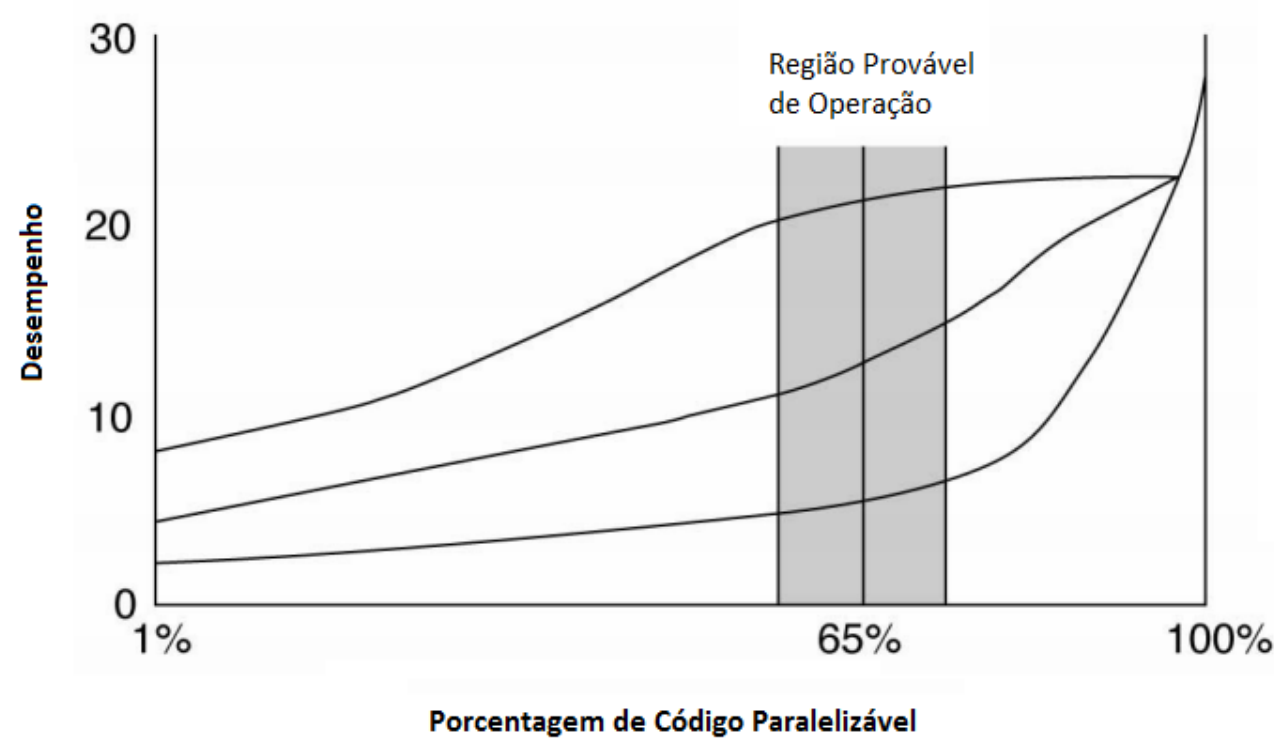

Figura 2.7: Lei de Amdahl (Amdahl, 1967)

\subsubsection{Eficiência}

Razão entre o speedup e o número de processadores:

$$
E_{p}=S_{p} / p=\frac{T_{1}}{p T_{p}}
$$

Estima quão bem os processadores estão sendo utilizados, tendo em vista o tempo gasto em sobrecarga como: sincronização e troca de mensagens.

\subsubsection{Escalabilidade}

É a capacidade do algoritmo de resolver um problema $n$ vezes maior em $n$ vezes mais processadores:

Escalabilidade $(p, n)=\frac{\text { Tempo para resolver um problema de tamanho } m \text { em } p \text { processadores }}{\text { Tempo para resolver um problema de tamanho } n m \text { em } n p \text { processadores }}$. 


\subsubsection{Taxa Sustentada de FLOPS}

A taxa sustentada de FLOPS (Floating-point Operations per Second) mede a capacidade de execução de operações de ponto flutuante por segundo e quão bem uma implementação específica explora a arquitetura alvo. Vale notar que essa métrica, apesar de ser comumente utilizada em HPC, não indica, necessariamente, que um algoritmo é eficiente. É fato que um algoritmo alternativo com menor taxa sustentada de FLOPS pode resolver um mesmo problema mais rapidamente. 


\section{Capítulo 3}

\section{Computação em GPU}

Nos últimos anos, a capacidade de hardware de processamento gráfico (GPU) teve um crescimento sem precedentes. As novas gerações de arquitetura de GPU possibilitam a programação de propósito geral enquanto mantêm alta banda de memória e grande poder computacional. Tal potencial de paralelismo é devido ao maior número de transistores dedicados a ULAs (Unidades Lógicas Aritméticas), em vez de controle de fluxo e cache, comparado com CPUs. Essa conformação é propícia para processamento massivamente paralelo. Porém requer maior conhecimento da arquitetura utilizada para aproveitamento da capacidade computacional. A figura 3.1 compara o crescimento de poder computacional de CPUs e GPUs.

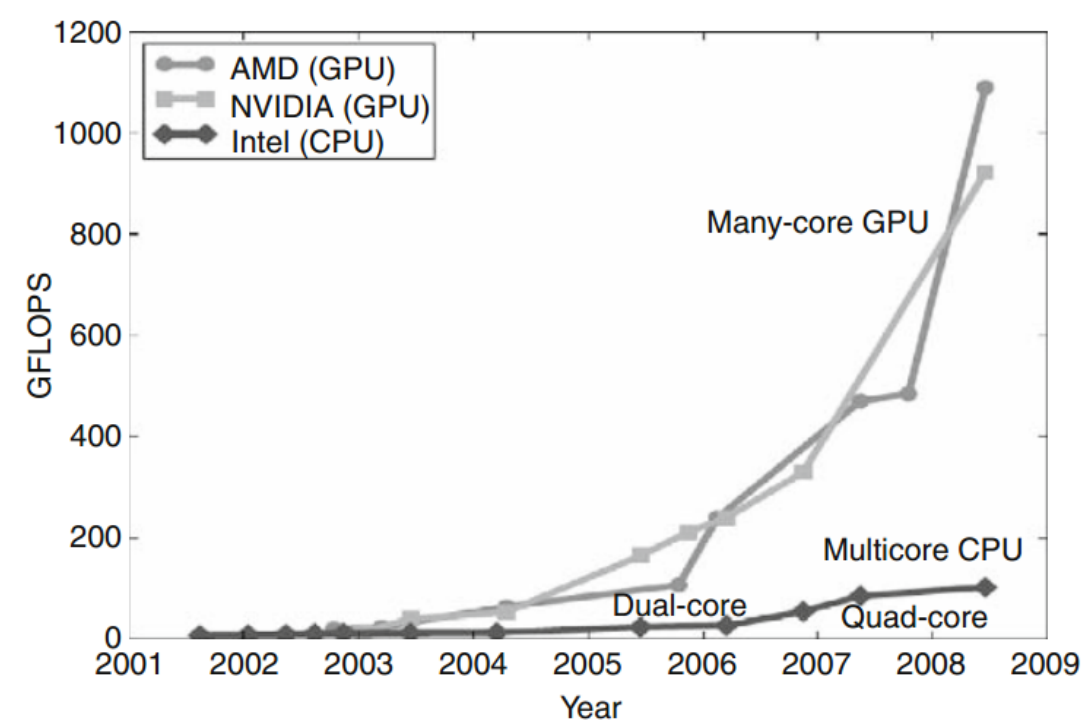

Figura 3.1: Poder computacional (GFLOP/s) CPU X GPU (Kirk e Hwu, 2010)

A figura 3.2 compara a utilização da área do chip entre CPU e GPU. A primeira arquitetura reserva maior área para controle de fluxo e cache e possui mais memória principal. Dessa forma, a CPU é mais apropriada para tarefas sequenciais. No caso da GPU, temos uma área maior dedicada para unidades lógicas aritméticas, o que resulta em maior capacidade de execução de operações de ponto flutuante. Dessa forma, essa arquitetura é mais adequada para tarefas com paralelismo de dados (modelo SIMD). 


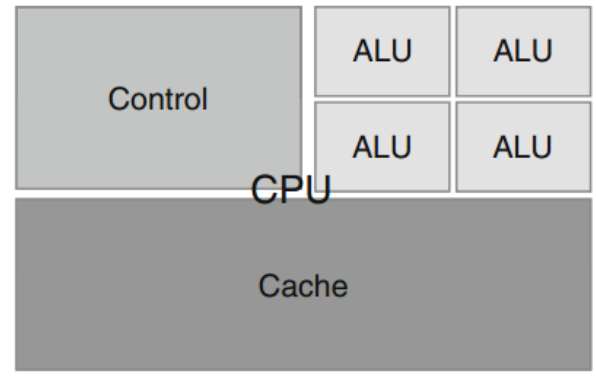

\section{DRAM}

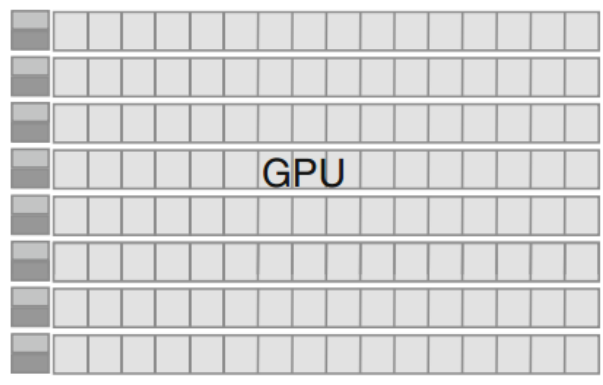

DRAM

Figura 3.2: Representação de Chip CPU X GPU (Kirk e Hwu, 2010)

A utilização de GPUs para computação de propósito geral é conhecida como GPGPU (General Purpose Computing GPU). Esse paradigma teve seu início com o advento de APIs gráficas como HLSL e Cg (Fernando, 2003), que trouxeram maior flexibilidade na programação. Contudo, ainda havia muitas dificuldades nessa abordagem, dado que o programador precisava modelar o problema manipulando estruturas de computação gráfica. Em 2006, a NVIDIA introduziu a arquitetura CUDA (Compute Unified Device Architecture), que propiciou um nível de abstração adequado para GPGPU, já que o programador não precisava mais utilizar a API gráfica e poderia desenvolver a aplicação em uma linguagem de mais alto nível.

Assim, neste capítulo apresentamos uma visão geral de uma arquitetura moderna de GPU, bem como uma introdução à plataforma de desenvolvimento a ser utilizada nesse trabalho.

\subsection{Arquitetura de GPU}

\subsubsection{Breve Evolução Histórica}

No começo dos anos 1990 não havia GPUs. Nesse período, controladores VGA (Video Graphics Array) geravam visualizadores gráficos 2D para PCs para acelerar interfaces gráficas. Em 1997, a NVIDIA lançou o RIVA 128, um acelerador gráfico 3D para jogos e aplicações de visualização tridimensional configuráveis via Microsoft Direct3D e OpenGL. A GeForce 256 foi a primeira GPU a ser lançada em 1999. Ela é um processador gráfico 3D de tempo real e pode ser configurada com OpenGL e APIs Microsoft DirectX (DX) 7.

Em 2001, a NVIDIA lançou a GeForce 3, que representou um marco na indústria por se tratar da primeira GPU programável no mercado. Em seguida, houve o lançamento das versões GeForce FX e GeForce 6800, que eram programáveis via Cg, além de DX9 e OpenGL. Esses processadores eram altamente paralelizáveis e sua arquitetura facilitou implementações em GPU com diferentes números de núcleos.

Além de renderizar gráficos em tempo real, programadores também utilizavam Cg para simulações físicas e computação de propósito geral em GPU. Esses primeiros programas de GPGPU já alcançavam alto desempenho, contudo eram de difícil escrita, pois programadores tinham que expressar cálculos com elementos não gráficos utilizando APIs gráficas como OpenGL.

A GeForce 8800, introduzida em 2006, foi outro marco na indústria de placas gráficas ao apresentar a primeira GPU de arquitetura gráfica unificada. Isso quer dizer que o programador poderia utilizar a GPU para cálculos de propósito geral sem usar estruturas gráficas específicas. Essa placa é capaz de executar eficientemente até 12.228 threads concorrentemente em 128 núcleos de processadores. Além disso, ela adicionou suporte à linguagem $\mathrm{C}$ de programação e outras linguagens de propósito geral. A NVIDIA disponibilizou essa arquitetura escalável em uma família de GPUs GeForce com diferentes números de núcleos de processadores para cada segmento de mercado particular. 
Até esse período, usuários construíam supercomputadores pessoais ao montar agregados compostos por nós de PCs com múltiplas GPUs. Em resposta a essa demanda, em 2007, a NVIDIA lançou a série Tesla C870, D870 e S870, que era composta por agregados de GPU baseados na GeForce 8800 .

Em 2009 foi lançada a geração Fermi de GPUs NVIDIA. Essa é a arquitetura alvo desse trabalho e será vista com maiores detalhes na seção seguinte. A figura 3.3 resume a evolução história de placas gráficas NVIDIA, enquanto que a figura 3.4 apresenta a evolução das APIs nesse período.

\begin{tabular}{|c|c|c|c|c|}
\hline \multirow[b]{2}{*}{ Date } & \multirow[b]{2}{*}{ Product } & \multicolumn{3}{|c|}{ Table 1. NVIDIA GPU technology development. } \\
\hline & & Transistors & CUDA cores & Technology \\
\hline 1997 & RIVA 128 & 3 million & - & 3D graphics accelerator \\
\hline 1999 & GeForce 256 & 25 million & - & First GPU, programmed with DX7 and OpenGL \\
\hline 2001 & GeForce 3 & 60 million & - & $\begin{array}{l}\text { First programmable shader GPU, programmed } \\
\text { with DX8 and OpenGL }\end{array}$ \\
\hline 2002 & GeForce FX & 125 million & - & $\begin{array}{l}\text { 32-bit floating-point (FP) programmable GPU with } \\
\text { Cg programs, DX9, and OpenGL }\end{array}$ \\
\hline 2004 & GeForce 6800 & 222 million & - & $\begin{array}{l}\text { 32-bit FP programmable scalable GPU, GPGPU } \\
\text { Cg programs, DX9, and OpenGL }\end{array}$ \\
\hline 2006 & GeForce 8800 & 681 million & 128 & $\begin{array}{l}\text { First unified graphics and computing GPU, } \\
\text { programmed in C with CUDA }\end{array}$ \\
\hline 2007 & Tesla T8, C870 & 681 million & 128 & $\begin{array}{l}\text { First GPU computing system programmed in C } \\
\text { with CUDA }\end{array}$ \\
\hline 2008 & GeForce GTX 280 & 1.4 billion & 240 & $\begin{array}{l}\text { Unified graphics and computing GPU, IEEE FP, } \\
\text { CUDA C, OpenCL, and DirectCompute }\end{array}$ \\
\hline 2008 & Tesla T10, S1070 & 1.4 billion & 240 & $\begin{array}{l}\text { GPU computing clusters, 64-bit IEEE FP, 4-Gbyte } \\
\text { memory, CUDA C, and OpenCL }\end{array}$ \\
\hline 2009 & Fermi & 3.0 billion & 512 & $\begin{array}{l}\text { GPU computing architecture, IEEE 754-2008 FP, } \\
64 \text {-bit unified addressing, caching, ECC } \\
\text { memory, CUDA C, C++, OpenCL, and } \\
\text { DirectCompute }\end{array}$ \\
\hline
\end{tabular}

Figura 3.3: Evolução Arquitetura GPU NVIDIA (Dally e Nickolls, 2010)

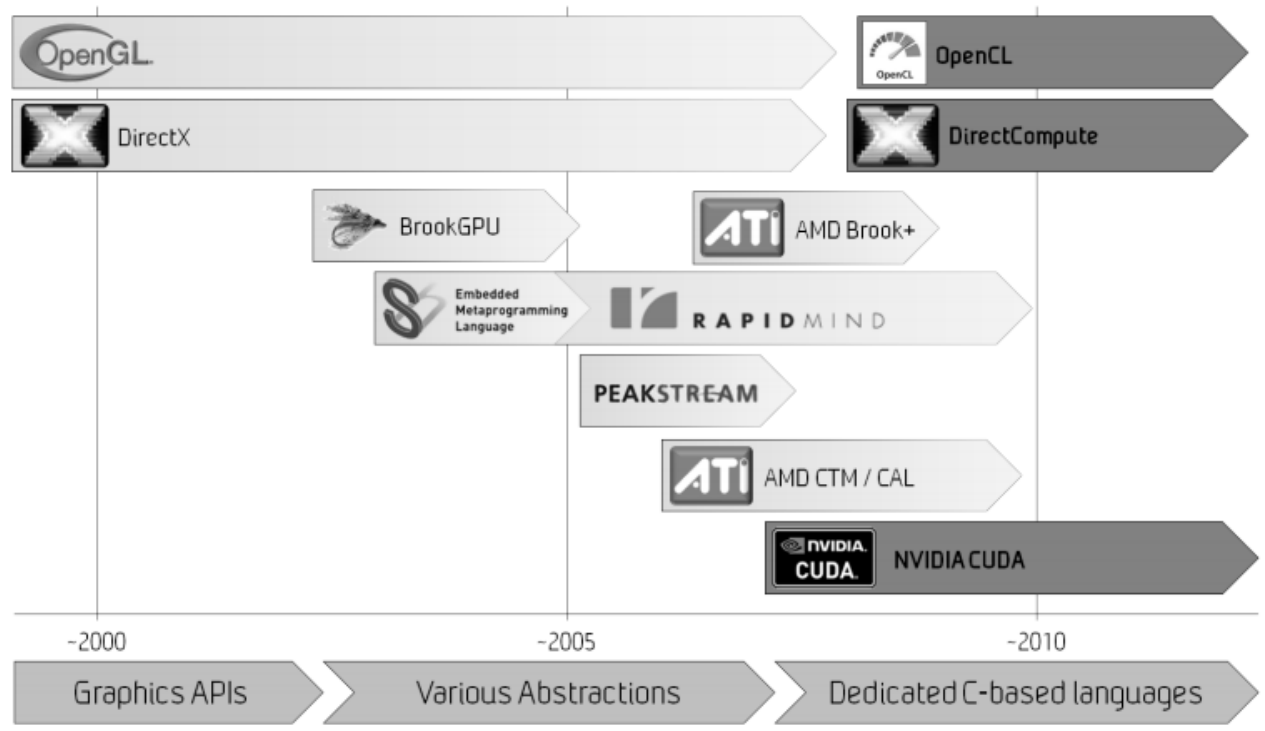

Figura 3.4: Evolução APIs para GPU (Brodtkorb et al., 2012) 


\subsubsection{Fermi}

A arquitetura Fermi implementa 3 bilhões de transistores com um total de 512 núcleos CUDA. Um núcleo CUDA corresponde à menor unidade de computação da GPU e executa uma instrução de ponto flutuante ou inteiro por clock $^{1}$ para uma thread. Como podemos ver na figura 3.5, os 512 núcleos CUDA são organizados em 16 unidades chamadas de Streaming Multiprocessor (SM), que contem 32 núcleos CUDA cada. A GPU tem seis partições de memória de 64-bits, totalizando uma interface de memória de 384-bits, que suporta até 6GB de memória GDDR5 DRAM. Uma interface conecta a GPU e sua memória (device) com a CPU e sua memória (host) via PCIe. O componente Giga Thread é o escalonador que distribui blocos de threads ao longo dos SMs. A figura 3.6 apresenta a configuração geral de um SM e de um núcleo CUDA. Cada um dos 32 núcleos CUDA de um SM contem uma ULA e uma UPF (Unidade de Ponto Flutuante).

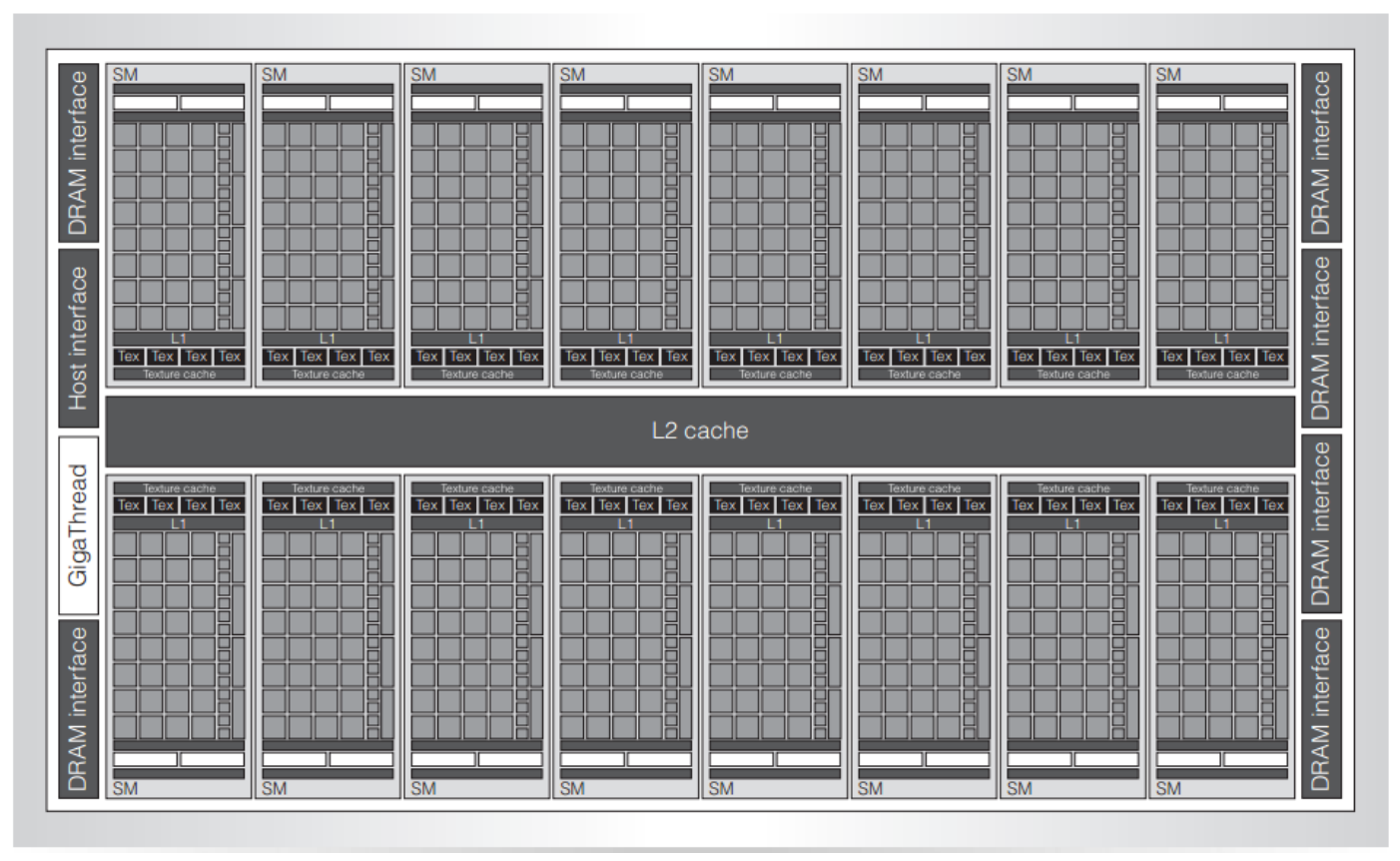

Figura 3.5: Arquitetura Fermi (Dally e Nickolls, 2010)

GPUs de gerações anteriores utilizavam o padrão IEEE 754-1985 (IEEE Task P754, 1985) para aritmética de ponto flutuante, ao passo que Fermi implementa o novo padrão IEEE 754-2008 (IEEE Task P754, 2008), que fornece maior precisão para operações de ponto flutuante de precisão dupla. Nesse novo padrão, foi disponibilizada a instrução FMA (Fused Multiply-Add ${ }^{2}$ ). Ela otimiza a instrução clássica MAD (Multiplicação-Adição) ao executar a multiplicação e adição com um passo adicional de arredondamento no final, de tal forma que não se perca precisão em truncamento. Assim, FMA é mais precisa do que se as operações fossem executadas de modo separado. A figura 3.7 demonstra o comportamento dessa instrução e a figura 3.8 exemplifica o ganho de desempenho da arquitetura Fermi em operações de dupla precisão em comparação com a geração anterior de GPU.

Cada SM possui 16 unidades de carga/armazenamento ${ }^{3}$ (LD/ST), isso permite que endereços de origem e destino sejam calculados para dezesseis threads por clock. Unidades de Funções Especiais $^{4}$ (SFUs) executam instruções transcendentais como seno ou cosseno . Cada SFU executa uma instrução por thread a cada clock.

\footnotetext{
${ }^{1}$ Indica uma unidade de tempo de execução.

${ }^{2}$ em português: multiplicação-adição fundidas

${ }^{3}$ no original: load/store

${ }^{4}$ no original: Special Function Units
} 


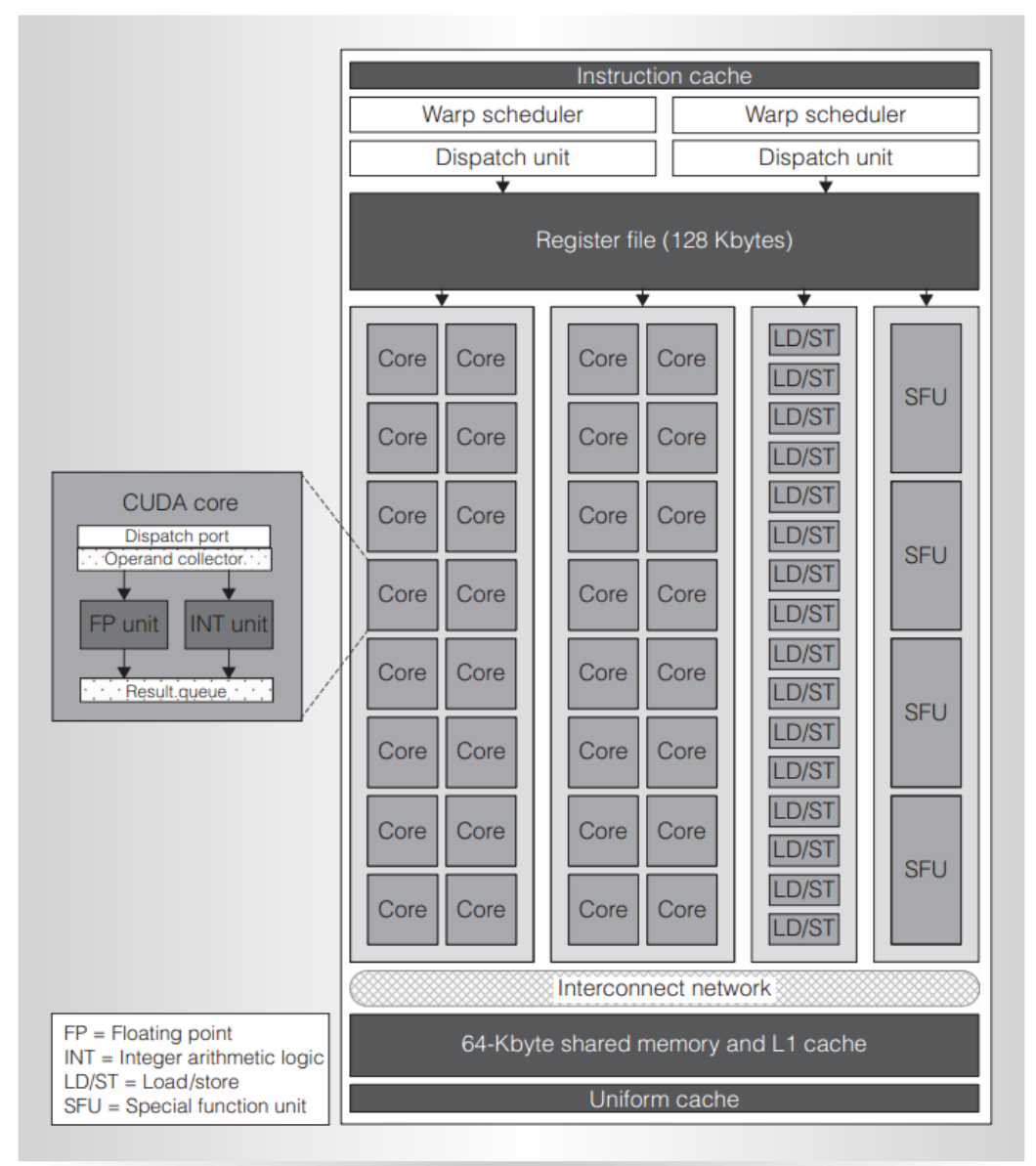

Figura 3.6: Streaming Multiprocessor Fermi (Dally e Nickolls, 2010)

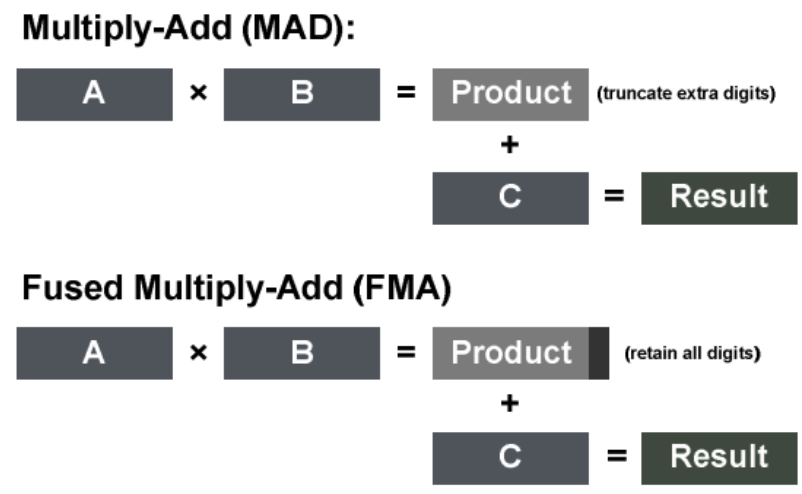

Figura 3.7: Instrução FMA (NVIDIA, 2009a)

O SM agenda threads para execução em grupos de 32 threads paralelas chamados de warps. Como cada SM possui dois escalonadores de warp, então é possível executar até 2 warps de maneira concorrente. O escalonador Fermi seleciona, então, dois warps e envia instruções de cada um para um grupo de 16 núcleos CUDA, dezesseis unidades LD/ST e quatro SFUs. Como os warps executam de maneira independente, o agendador da Fermi não precisa checar dependências entre instruções. A figura 3.9 demonstra esse funcionamento, que pode ser classificado como uma arquitetura SIMD. 


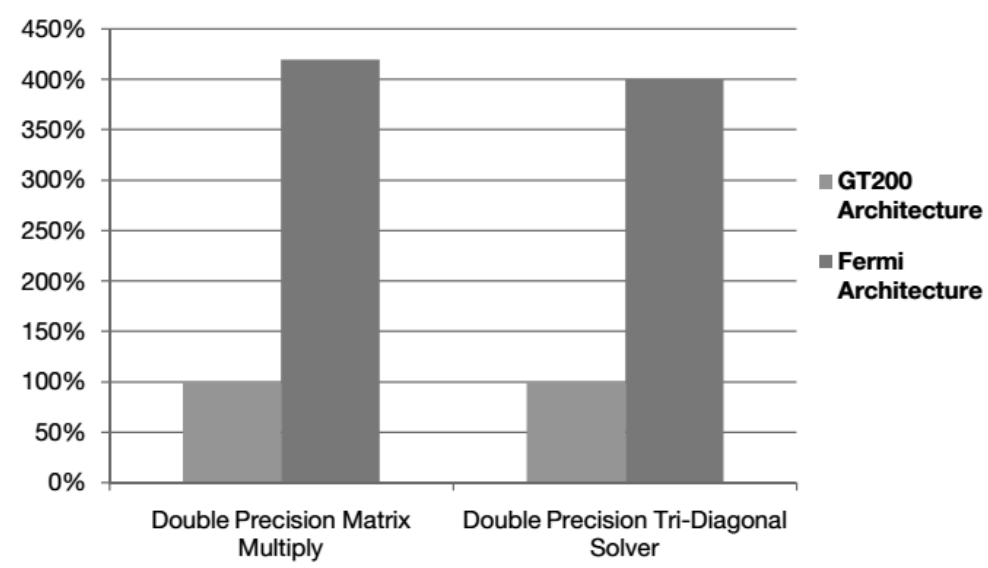

Figura 3.8: Exemplo de desempenho Fermi em aplicação de dupla precisão (NVIDIA, 2009a)

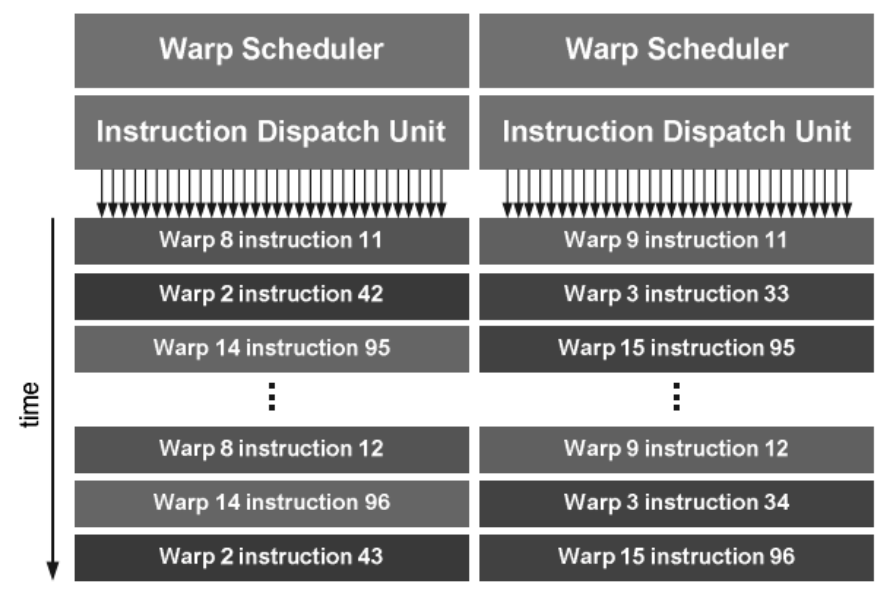

Figura 3.9: Agendador Fermi de warps (NVIDIA, 2009a)

Adicionalmente à memória compartilhada, a arquitetura Fermi possui dois níveis de memória cache. O cache L1 é individual ao SM e é configurável para suportar tanto memória compartilhada quanto cache das memórias local ou global. Essa memória de $64 \mathrm{~KB}$ posse ser configurada tanto com 48KB de memória compartilhada com 16KB de cache L1 quanto com 16KB de memória compartilhada com 48KB de cache L1. Aplicações que utilizam memória compartilhada com frequência podem tirar grande proveito da primeira configuração, enquanto que a segunda alternativa mostra-se muito útil em aplicações que tem acessos com pouca previsibilidade. Para um estudo de algoritmos eficientes em utilização de cache em GPU veja (Govindaraju e Manocha, 2007).

Além de grandes avanços em questões de desempenho, Fermi introduziu uma nova característica às placas modernas da NVIDIA: proteção de integridade de dados. Com o suporte a ECC (Error Correcting Code), essa arquitetura se tornou capaz de corrigir erros de único bit e detectar erros de dois bits em memória DRAM, caches L1 e L2, e registradores. Essa característica mostra-se de importância para grandes aplicações de HPC, que integram centenas de GPUs em um único sistema.

A NVIDIA classifica a evolução da tecnologia de GPU em compute capability. Como a arquitetura Fermi corresponde à compute capability 2.x, outras especificações técnicas podem ser vistas no apêndice A.1. Na tabela 3.1 sumarizamos as principais características da arquitetura Fermi vistas até aqui. 


\begin{tabular}{|l||c|}
\hline \multicolumn{1}{|l||}{ Transistores } & 3 bilhões \\
\hline Máximo Núcleos CUDA & 512 \\
\hline Ponto flutuante de dupla precisão & 256 FMA operações / clock \\
\hline Ponto flutuante de precisão simples & 512 FMA operações / clock \\
\hline Unidades de Funções Especiais & 4 \\
\hline Escalonador de warp por SM & 2 \\
\hline Memória Compartilhada por SM & Configurável: $48 \mathrm{~KB} / 16 \mathrm{~KB}$ \\
\hline Cache L1 por SM & $48 \mathrm{~KB} / 16 \mathrm{~KB}$ \\
\hline Cache L2 & $768 \mathrm{~KB}$ \\
\hline Suporte a ECC & $\mathrm{Sim}$ \\
\hline
\end{tabular}

Tabela 3.1: Sumário de Características Arquitetura Fermi

\subsection{CUDA}

CUDA é uma arquitetura de computação paralela para propósito geral. Por meio dessa, a GPU pode ser acessada como um conjunto de multiprocessadores (SMs) que são capazes de executar um grande números de threads. Atualmente, diversas APIs suportam CUDA, como: C, C++, Java e Fortran (vide figura 3.10). Este trabalho baseia-se na API de CUDA para C (CUDA C).

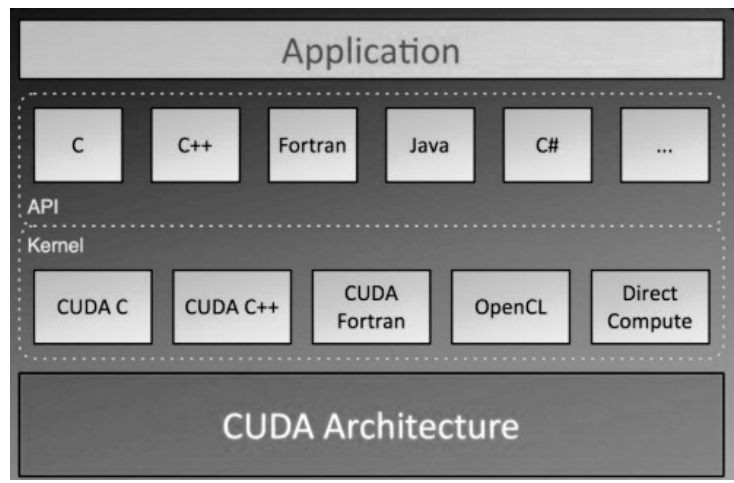

Figura 3.10: CUDA API (NVIDIA, 2011a)

\subsubsection{Modelo de Programação}

Uma função executada em GPU é chamada de kernel. O conjunto de threads de um kernel define uma grade ${ }^{5}$ que, por sua vez, é subdividida em blocos de threads. Todas as threads em uma mesma grade executam o mesmo kernel. Com isso, para distinção do endereçamento, CUDA define as seguintes variáveis de sistema:

- blockIdx: índice do bloco

- threadIdx: índice da thread

- gridDim: dimensão da grade

- blockDim: dimensão dos blocos

Uma grade é bidimensional, portanto o índice de bloco é constituído pelo par (blockIdx.x, blockIdx.y), ao passo que o índice de thread pode ter até três dimensões (threadIdx.x, threadIdx.y,

${ }^{5}$ no original: grid 
threadIdx.z). A figura 3.11 representa tal indexação em uma organização bidimensional de blocos e threads.

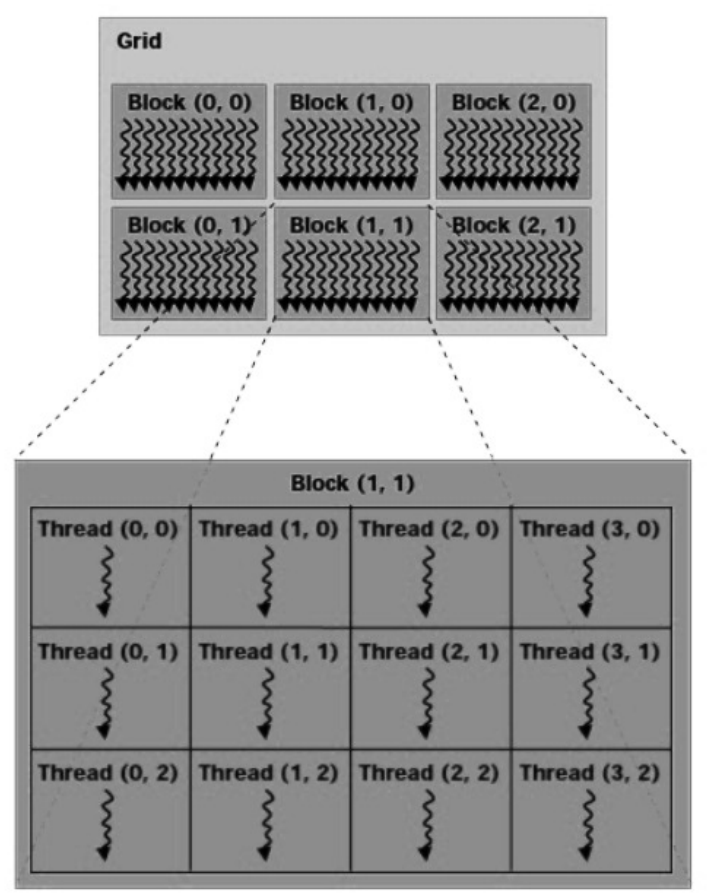

Figura 3.11: Hierarquia de threads (NVIDIA, 2011a)

Um kernel é definido pela extensão __ global ___ e sua configuração deve determinar o número de blocos na grade (numBlocos) e de threads por bloco (numThreads) de sua composição. Tal configuração possui a seguinte sintaxe: $<<<$ numBlocos, numThreads $>>>$. O código 3.1 exemplifica o lançamento de um kernel com um bloco e $N$ threads responsável por somar dois vetores de $N$ elementos.

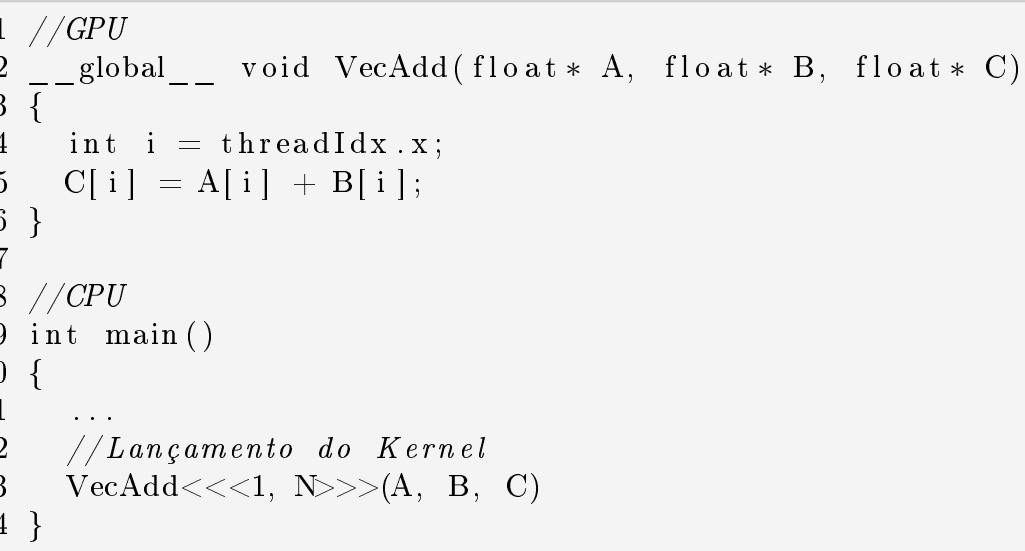

Código 3.1: Exemplo kernel

CUDA também dispõe de outras duas extensões de função: ${ }_{-}$device ${ }_{-}{ }^{\mathrm{e}}{ }_{-}{ }^{\text {host }}{ }_{-}$. A tabela 3.2 sumariza a utilização das mesmas. 


\begin{tabular}{|c|c|c|}
\hline Função & Executado em & Possível de ser chamado em \\
\hline device $\quad$ float DeviceFunc() & device & device \\
\hline global ${ }^{--}$void KernelFunc() & device & host \\
\hline _host__float HostFunc() & host & host \\
\hline
\end{tabular}

Tabela 3.2: Extensões de função em CUDA

\subsubsection{Modelo de Memória}

A hierarquia de threads enunciada incorre em diferentes tipos possíveis de memória e em formas distintas de utilização da mesma. Desses tipos, podemos enunciar:

- Registradores: são rápidos, porém escassos. Cada thread possui um conjunto privado de registradores.

- Memória Compartilhada ${ }^{6}$ : threads em um mesmo bloco compartilham um espaço de memória, o qual funciona como um cache manipulado explicitamente pelo programa.

- Memória Local ${ }^{7}$ : cada thread possui acesso a um espaço de memória local, além de seus registradores. Essa área de memória está fora do micro-chip de processamento, junto à memória global, e portanto, ambas essas memórias possuem tempos de acessos similares.

- Memória Global ${ }^{8}$ : esta memória está disponível para todas as threads em cada bloco e em todas as grades. Trata-se da única maneira de threads de diferentes blocos colaborarem.

A tabela 3.3 sumariza as características dos diferentes tipos de memória enunciados.

\begin{tabular}{|l|c|c|c|c|}
\hline Declaração da Variável & Tipo de Memória & Local & Escopo & Tempo de vida \\
\hline \hline int var & Registrador & No chip & Thread & Thread \\
int var[10] & Memória Local & Fora do chip & Thread & Thread \\
_-_shared__int var & Memória Compartilhada & No chip & Bloco & Bloco \\
__device__int var & Memória Global & Fora do chip & Grade & Aplicação \\
\hline
\end{tabular}

Tabela 3.3: Tipos de Memória

\footnotetext{
${ }^{6}$ no original: Shared Memory

${ }^{7}$ no original: Local Memory

${ }^{8}$ no original: Global Memory
} 


\section{Capítulo 4}

\section{Técnicas de Otimização de Desempenho em CUDA}

A programação de centenas ou até milhares de núcleos de processamento pode se mostrar como um grande desafio. Contudo, otimizar o desempenho desses programas em uma arquitetura de GPU moderna pode ser um problema ainda maior. Assim, este capítulo tem o objetivo de apresentar boas práticas de utilização da arquitetura CUDA. Ao final, dispomos um sumário das melhores práticas de otimização de desempenho classificadas por ordem de prioridade.

\subsection{Medição Tempo em CUDA}

Podemos medir tempos de execução em CUDA utilizando tanto métodos tradicionais de CPU quanto métodos específicos de GPU. Entretanto, há alguns pontos a considerar nessas escolhas. Primeiro, é importante notar que chamadas à API CUDA podem ser assíncronas. Nesse caso, é necessário garantir a sincronia de threads ao utilizar temporizadores em CPU. Além disso, deve-se tomar cuidado ao criar pontos de sincronização em CPU. Isso pode causar paradas em GPU.

Ao utilizar temporizadores de CPU, para medir corretamente o tempo transcorrido em uma chamada ou sequência de chamadas em CUDA, é necessário sincronizar a thread da CPU com a GPU com a instrução cudaThreadSynchronize() imediatamente antes e depois de iniciar o temporizador na CPU. No código 4.1 exemplificamos esse tipo de medição. Medições realizadas com temporizadores de GPU comumente utilizam a biblioteca GPU Events. No código 4.2 demonstramos a utilização dessa biblioteca.

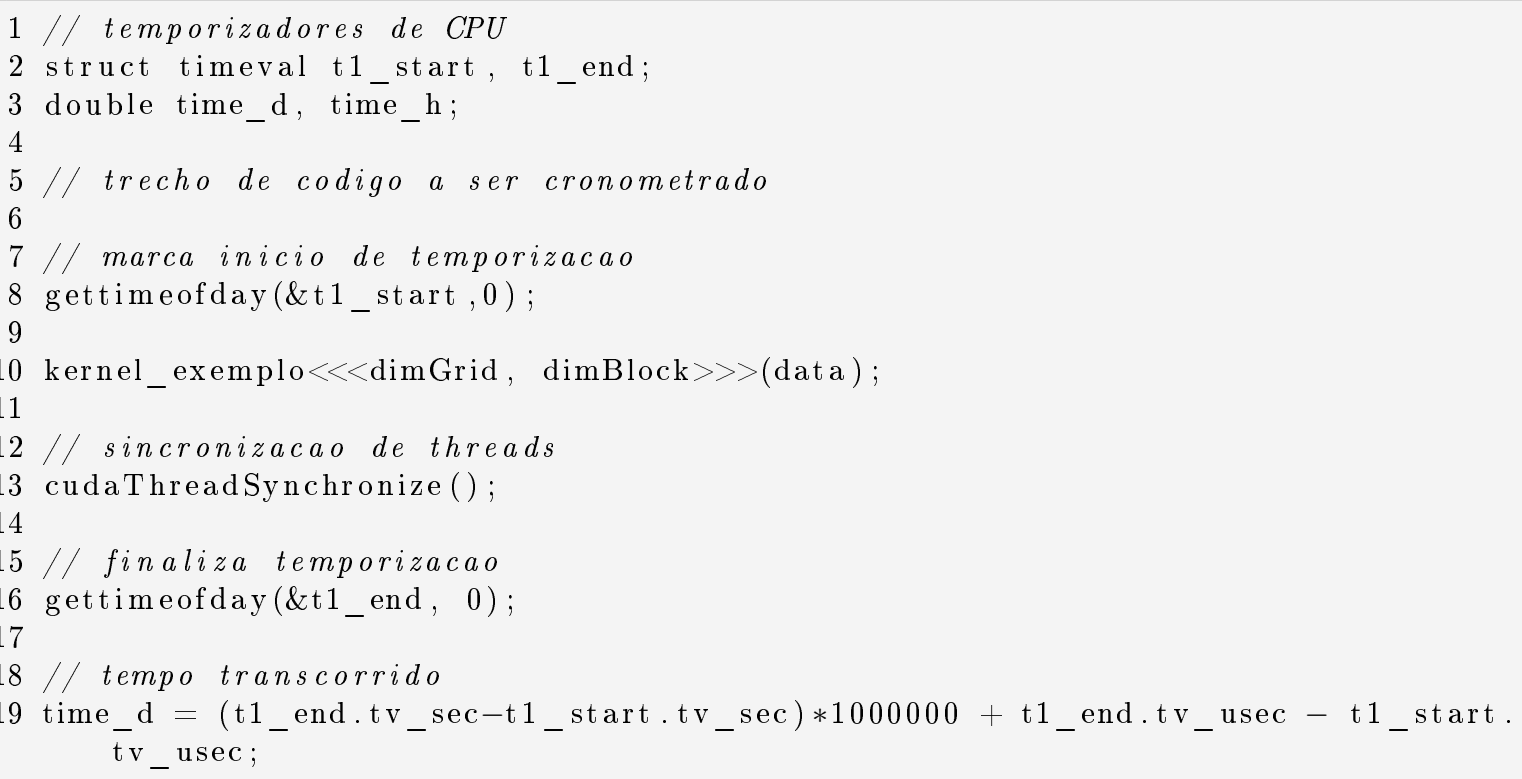

Código 4.1: Temporizador $C P U$ 


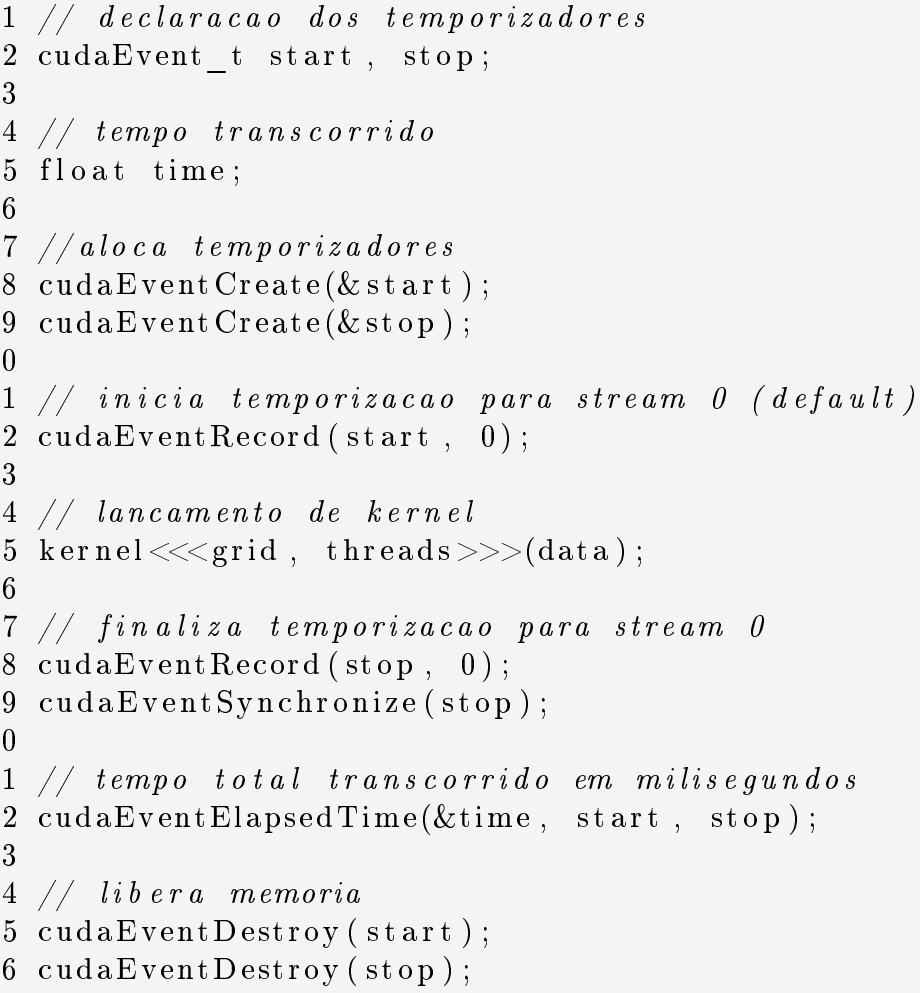

Código 4.2: Temporizador GPU Events

\subsection{Execução Concorrente Assíncrona}

Para facilitar a execução concorrente entre host e device, algumas chamadas de função são assíncronas: o controle é retornado para thread do host antes que o device complete as tarefas requisitadas.

Dois tipos de execução concorrente são particularmente importantes:

- Sobreposição em transferência de dados

- Execução de Kernels paralelos

Para verificar se o device permite tais tipos de concorrência, deve-se executar a chamada cudaGetDeviceProperties() e checar os atributos deviceOverlap e concurrentKernels.

\subsubsection{Comunicação Host-Device}

Ao alocar memória em CPU que vai ser utilizada para transferir dados para GPU, há dois tipos de memória possíveis: memória pinada ${ }^{1}$ e memória não pinada ${ }^{2}$. A primeira possibilita transferências via PCIe mais rápidas com cópias assíncronas de memória. Seu uso se dá pelas funções cudaHostAlloc e cudaFreeHost no lugar das funções de CPU malloc e free, respectivamente.

Apesar da utilização de memória pinada poder acelerar as transferências via PCIe, essa memória não deve ser super utilizada, pois há considerável sobrecarga em sua utilização e seu uso não planejado pode causar perda de desempenho. Assim, sua quantidade ótima depende da aplicação específica.

\footnotetext{
${ }^{1}$ no original: pinned memory

${ }^{2}$ no original: non-pinned memory
} 


\subsubsection{Kernels Paralelos}

Em compute capability 1.x, o único modo de se utilizar todos os multiprocessadores é lançar um único kernel com pelo menos o número de blocos igual ao de SMs. A partir da compute capability 2.x, CUDA permite a execução de múltiplos kernels. Tal execução é feita utilizando CUDA streams. No código 4.3 exemplificamos a declaração de streams para sobreposição entre transferência de dados e computação em GPU. Nas linhas 2 e 3 dois streams são criados para explicitar independência de execução. Na linha 5 é feita uma cópia assíncrona de dados para GPU e na linha 6 o kernel é lançado em um stream diferente e, portanto, executado de forma paralela com a transferência de dados.

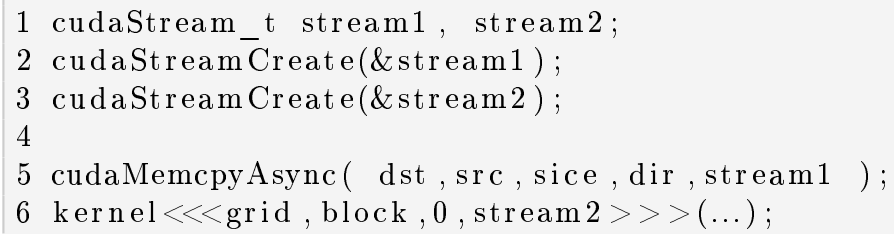

Código 4.3: CUDA streams para sobreposição entre transferência de dados e computação em GPU

O código 4.4 fornece um exemplo de execução concorrente entre kernels. Ele realiza os seguintes passos:

1. Cria streams para explicitar inter-independencia

2. Aloca memória pinada

3. Associa cópia de dados e execução de kernels com seus respectivos streams

4. Desaloca memória utilizada

Entre as linhas 1 e 8 dois streams são definidos ao criar objetos do tipo cudaStream e um vetor de floats é alocado em memória pinada. Entre as linhas 10 e 16 cada stream: copia sua porção de dados do vetor hostPtr para o vetor inputDevPtr em memória de device; processa de modo concorrente inputDevPtr no device ao chamar MyKernel(); copia o resultado obtido em outputDevPtr de volta para o host em hostPtr. Ao final, na linha 18, cada stream é liberado com a chamada de cudaStreamDestroy.

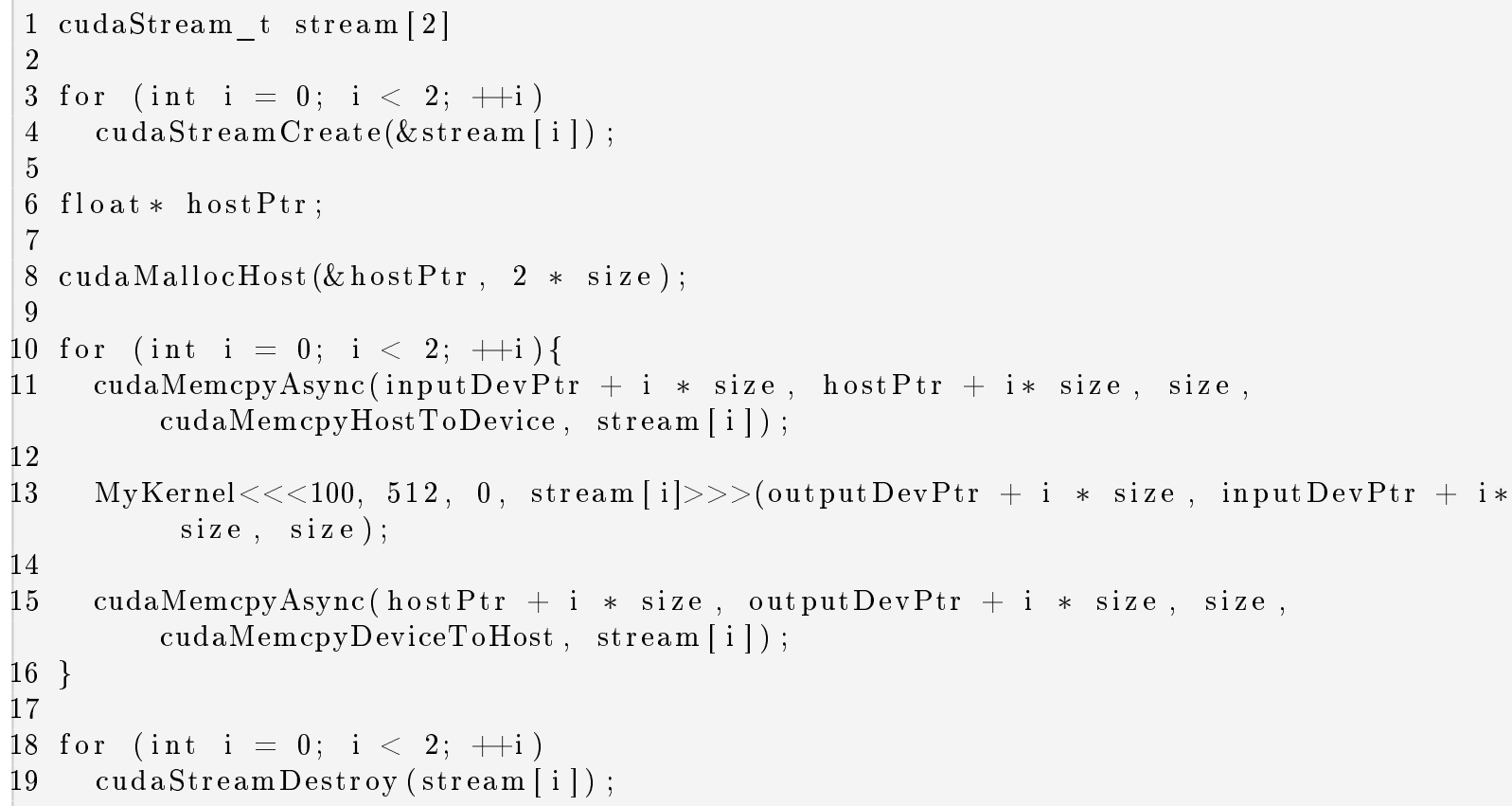

Código 4.4: Exemplo de execução concorrente entre kernels 
Um stream é uma sequência de comandos que são executados em ordem. Não há ordenação entre comandos de diferentes streams. Associar diferentes streams a diferentes operações significa explicitar independência dessas. Isso possibilita intervalar operações. Dessa forma, um lançamento de kernel e uma cópia de memória podem ter sobreposição. No código 4.5 apresentamos um exemplo de divisão de cópia de dados e execução de kernel em passos intermediários para esconder a latência. A figura 4.1 ilustra tal comportamento.

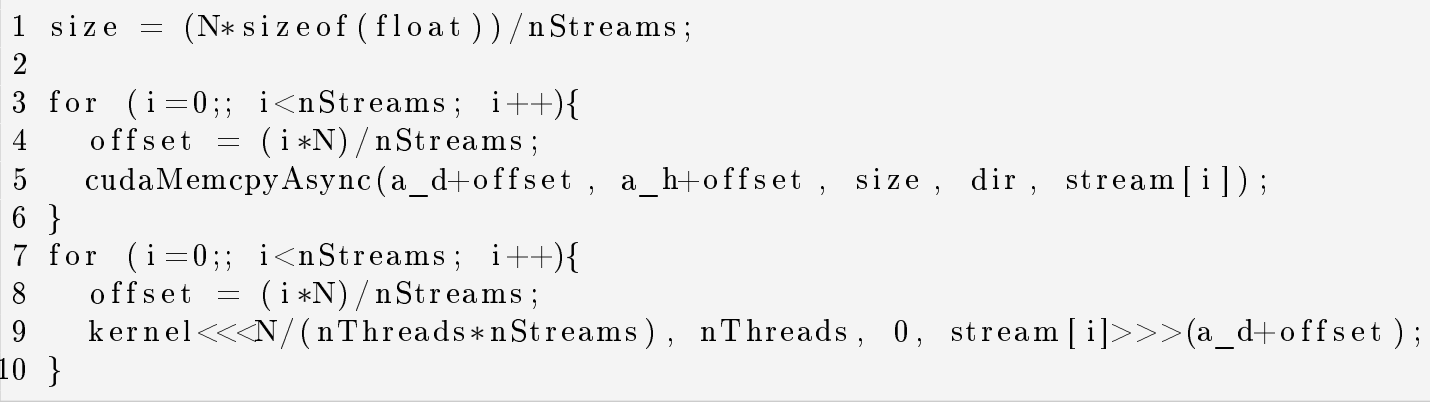

Código 4.5: Exemplo de divisão de cópia de dados e execução de kernel

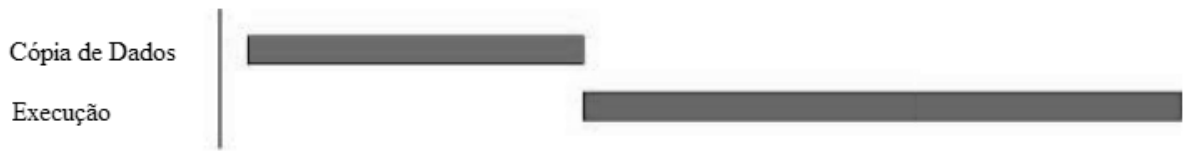

(I)

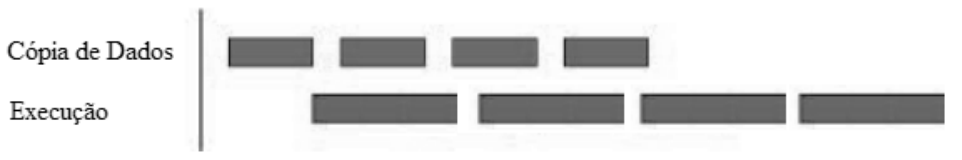

(II)

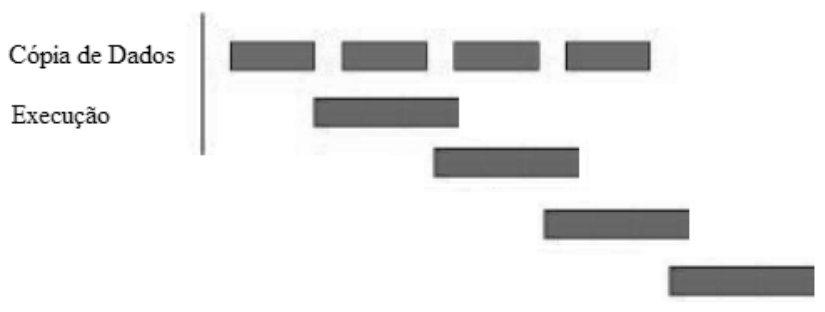

(III)

Figura 4.1: (I) Execução e cópia sequenciais; (II) Execução e cópia paralelos em compute capability 1.x; (III) Execução e cópia paralelos em compute capability 2.x; 


\subsection{Otimizações de Memória}

\subsubsection{Coalesced Memory}

Uma das mais importantes considerações de otimização na arquitetura CUDA é Coalesced Memory. Em um dispositivo de compute capability 2.x, os acessos à memória global realizados por threads dentro de um mesmo warp são reunidos em uma mesma transação de memória quando alguns requisitos de acesso são satisfeitos. Para entendê-los, devemos ver a memória global em termos de segmentos alinhados de 32 palavras de memória. Assim, um padrão de acesso de coalesced memory acontece quando a $k$-ésima thread acessa a $k$-ésima palavra de um segmento, com a observação que nem todas as threads precisam participar do acesso. A Figura 4.2 ilustra tal situação.

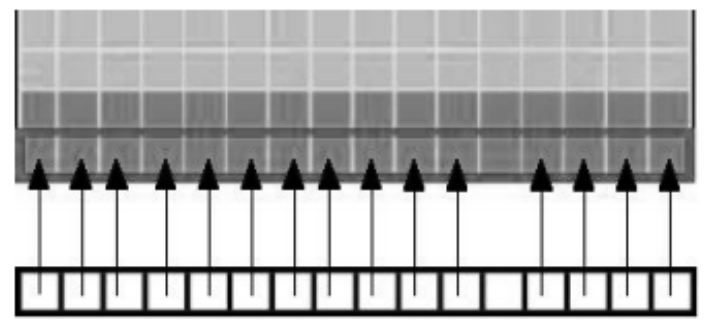

Figura 4.2: Acesso Coalesced Memory (NVIDIA, 2011a)

\subsubsection{Memória Compartilhada}

Como a memória compartilhada é interna ao chip, ela é muito mais rápida que a memória local e a memória global. De fato, tal memória tem latência, no mínimo, 100x menor que a memória global, quando não há nenhum conflito de bancos de memória entre as threads (NVIDIA, 2010b).

Para alcançar banda de memória máxima para acessos paralelos, a memória compartilhada é dividida em módulos (bancos) de igual tamanho que podem ser acessados simultaneamente. Assim, quaisquer leituras ou escritas em $n$ endereços distribuídos por $n$ bancos distintos de memória são servidos ao mesmo tempo, correspondendo em uma banda efetiva $n$ vezes maior que a banda de um único banco.

Entretanto, se diferentes endereços de requisição de memória são mapeados para um mesmo banco, os acessos são serializados. O hardware divide tal requisição em sub-requisições sem conflitos de acesso, o que diminui a banda efetiva em fator igual ao número de sub-requisições. Para exemplificar essa sobrecarga dessa serialização, podemos criar um kernel (Che et al., 2008) que transpõe tanto colunas quanto linhas em paralelo em uma matriz $16 \mathrm{X} 16$. Ao transpor colunas, o kernel exibe um número maximal de conflitos de acesso a bancos de memória, enquanto que ao transpor linhas, não há conflitos. A figura 4.3 mostra que a existência de conflitos em bancos de memória compartilhada dobra, aproximadamente, o tempo de execução do kernel.

\subsubsection{Registradores}

Em geral, acesso a registradores consome zero ciclos de clocks extras por instrução, mas atrasos podem ocorrer devido a, por exemplo, conflitos em acessos a bancos de registro de memória. O compilador e o hardware escalonador de threads controlam as instruções tão ótimo quanto possível para evitar conflitos em bancos de memória. Contudo, para obter melhores resultados é necessário configurar o número de threads por bloco como um múltiplo de 64 (NVIDIA, 2010b).

\subsubsection{Memória Constante}

Há um total de $64 \mathrm{~KB}$ de memória constante tanto em dispositivos de compute capability 1.x quanto 2.x. Esse tipo de memória fornece ganho de desempenho, pois é mantida em cache. Assim, uma leitura de memória constante custa apenas uma leitura de memória do device. 


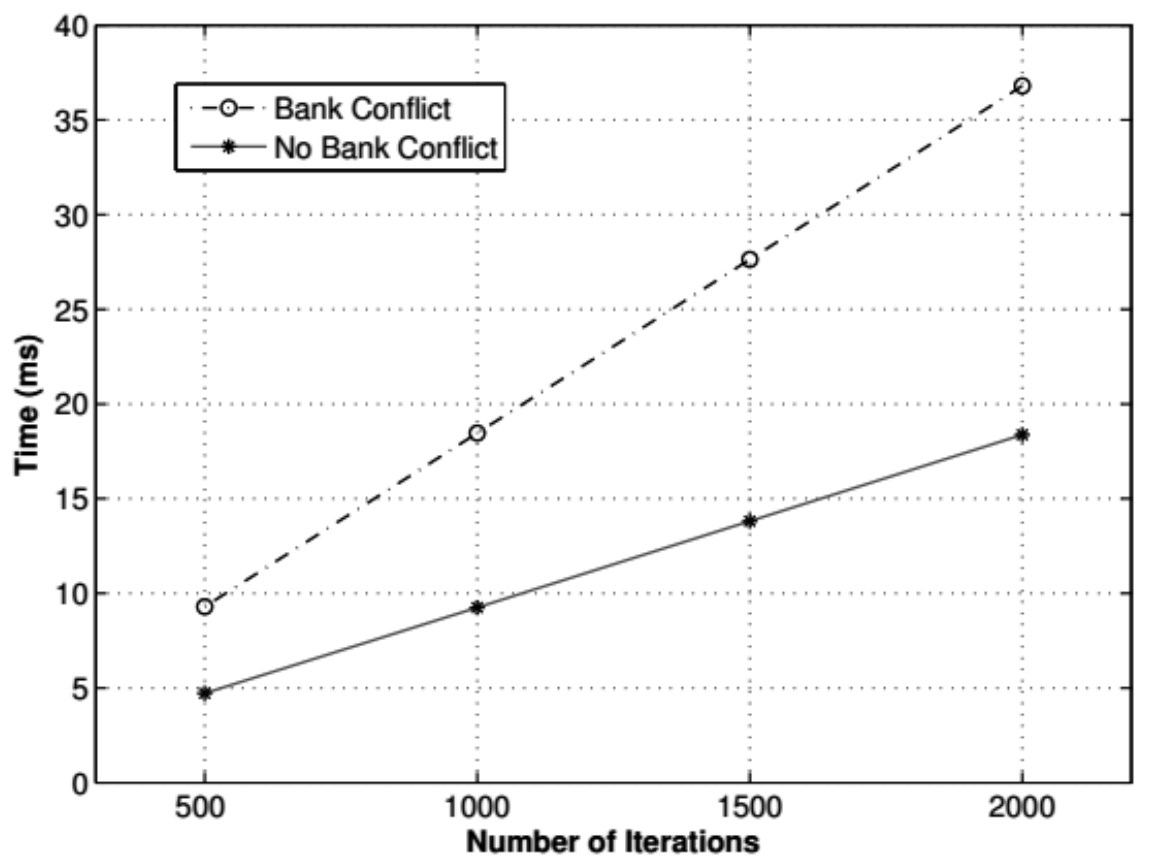

Figura 4.3: Sobrecarga em conflitos de banco em memória compartilhada (Che et al., 2008).

Para threads de um mesmo warp, ler memória constante é tão rápido quanto ler registradores, desde que todas as threads leiam do mesmo endereço. Acessos a diferentes endereços de memória por threads de um mesmo warp são serializados. Assim, o custo cresce linearmente com o número de endereços diferentes acessados. Alternativamente, dispositivos de compute capacility 2.x suportam a instrução LDU (LoaD Uniform), que o compilador utiliza para carregar variáveis que são somente leitura, não dependem de identificador de thread ou apontam para memória global.

\subsubsection{Memória Local}

É importante explicitar enganos comuns sobre a utilização de memória local. Essa memória tem esse nome devido ao seu escopo ser local à thread, não devido a sua localidade física. Na verdade, a memória local é externa ao chip. Assim, seu acesso tem custo da mesma ordem de grandeza que o acesso à memória global. Em compute capability 1.x, como em memória global, a memória local não é mantida em cache. Portanto, o a palavra "local" em seu nome não significa necessariamente acesso com maior desempenho.

\subsection{Controle de Fluxo Condicional}

Em CUDA, instruções de controle de fluxo condicional podem impactar significativamente a largura de banda de memória de instruções se threads dentro de um mesmo warp seguirem controles de fluxo diferentes. Ao executar caminhos de fluxo divergentes, todas as threads dentro de um mesmo warp executam cada instrução de cada caminho divergente, o que causa uma potencial perda de desempenho. Para obtenção de máximo desempenho em casos onde o controle de fluxo depende do identificador da thread, a condição de controle deve ser escrita visando à minimização de warps divergentes. A figura 4.4 de (Che et al., 2008) demonstra que a sobrecarga aumenta de linearmente com o aumento do número de threads divergentes. 


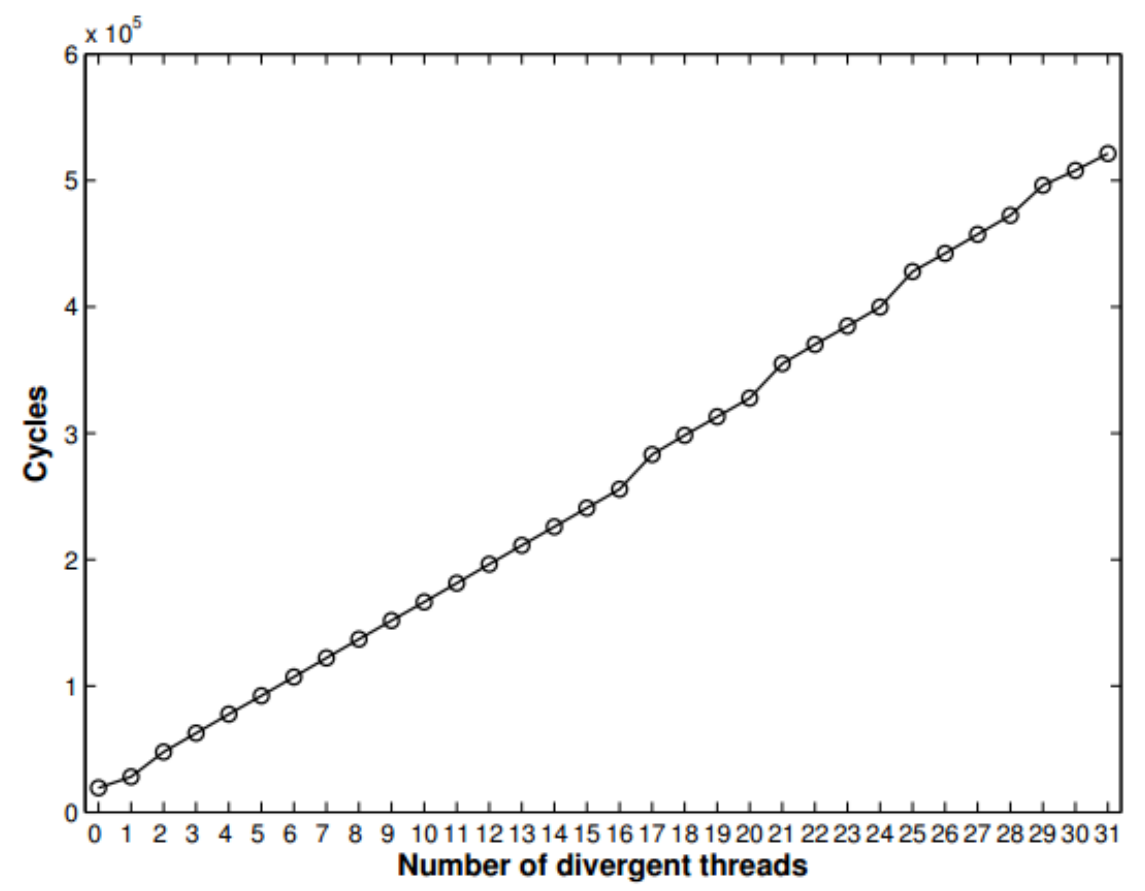

Figura 4.4: Sobrecarga em threads divergentes (Che et al., 2008).

\subsection{Configuração de Execução}

Um princípio para obtenção de bom desempenho é manter o multiprocessador do device tão ocupado quanto possível. Uma GPU na qual o trabalho é distribuido de forma desigual ao longo dos multiprocessadores em geral resulta em desempenho reduzido. Assim, é importante construir a aplicação para utilizar threads e blocos de forma a maximizar o uso do hardware e limitar práticas que impeçam a livre distribuição das instruções a executar. Para isso, dois conceitos têm papel fundamental: a ocupação do multiprocessador e o gerenciamento dos recursos alocados para uma atividade particular.

\subsubsection{Ocupação}

Instruções de uma mesma thread são executadas sequencialmente em CUDA. Por isso, executar outros warps quando um warp está pausado é a única forma de esconder latência e manter o hardware ocupado. Assim, uma métrica relacionada ao número de warps ativos em um multiprocessador é importante para determinar quão eficientemente o hardware está sendo utilizado. Para isso, uma métrica comumente utilizada é Ocupação ${ }^{3}$. Ela é definida como a razão entre o número de warps ativos por multiprocessador e o número máximo possível de warps ativos no mesmo.

Intuitivamente, Ocupação mede percentualmente a capacidade do hardware em processar warps ativos. A capacidade máxima de warps ativos em um multiprocessador pode ser obtida no apêndice A.1.

Com isso, é boa prática maximar a ocupação dos SMs. Como um auxílio nesse intuito, a NVIDIA fornece uma calculadora de Ocupação de fácil uso, disponível em (NVIDIA, 2010a). Contudo, tal estratégia é apenas uma heurística, dado que maior ocupação não significa, necessariamente, maior desempenho, já que outros recursos da placa gráfica podem se tornar fatores limitantes de desempenho, como comprovado em (Hong e Kim, 2009).

\footnotetext{
${ }^{3}$ no original: occupancy
} 


\subsubsection{Configuração de Registradores}

A disponibilidade de registradores é um dos fatores que determina a Ocupação. Alocação de registradores permite que threads mantenham suas variáveis locais mais próximas para um acesso de menor latência. Entretanto, registradores são recursos limitados que threads de um mesmo multiprocessador devem compartilhar.

Registradores são alocados em escopo de bloco. Assim, se cada bloco de threads utiliza muitos registradores, o número total de blocos permitido em um multiprocessador pode ser reduzido e, assim, a Ocupação pode ser prejudicada. O número máximo de registradores por threads pode ser definido em tempo de compilação utilizando a opção -maxrregcount. Veja (NVIDIA, 2011a) para fórmulas de alocação de registradores por compute capability e o apêndice A.1 para o número total de registradores disponíveis nesses dispositivos.

\subsection{Otimização de Instruções}

Tomar conhecimento de conjunto de instruções, frequentemente, permite otimizações de mais baixo nível, especialmente interessantes em trechos de código que são executados repetidas vezes. A seguir, discutimos instruções de mais baixo nível presentes na biblioteca CUDA.

\subsubsection{Bibliotecas Matemáticas}

CUDA fornece duas versões de funções em sua biblioteca matemática. Elas podem ser distinguidas pelo prefixo "_-" (por exemplo: nomeFuncao() e sua correspondente __nomeFuncao()). Funções que seguem a convenção __nomeFuncao() utilizam instruções de mais baixo nível. Elas são mais rápidas, contudo de menor precisão (e.g. _ _ $\operatorname{sinf}(\mathrm{x})$ e ___expf(x)). A opção de compilação use_fast_math converte funções do tipo nomeFuncao() em sua respectiva versão _ _nomeFuncao(). Assim, esse tipo de compilação deve ser executado sempre que precisão tiver menor prioridade que desempenho. O apêndice A.2 lista funções afetadas pelo uso de -use_fast_math.

\subsubsection{Instruções Aritméticas}

Instruções float de precisão simples fornecem melhor desempenho que instruções correspondentes de precisão dupla e, portanto, devem ser consideradas quando possível. Em (NVIDIA, 2011a) é apresentada a largura de banda de operações aritméticas por compute capability.

Dentre as instruções aritméticas, alguns usos devem ser evitados, como por exemplo operações de módulo ou divisão. Essas operações são particularmente custosas e seu uso deve ser controlado. Uma opção é a substituição por operações bit-a-bit (e.g. utilização de shift $i>>1$ no lugar de divisão $i / 2$ ). Conversões automáticas de tipos também devem ser evitadas, já que isso gera instruções a mais pelo compilador. Alguns exemplos são a utilização de char ou short, que são convertidas em int, ou a utilização de constantes de dupla precisão como argumentos em cálculos de precisão simples.

\subsection{Sumário de Melhores Práticas}

Como visto, técnicas de otimização de desempenho em CUDA baseiam-se em três estratégias fundamentais:

- Maximar a quantidade de código paralelizada

- Otimizar uso de memória para alcançar maior largura de banda possível

- Otimizar uso de instruções para alcançar maior quantidade de execução de operações por unidade de tempo

Na tabela 4.1 sumarizamos as principais boas práticas em CUDA vistas até aqui em ordem de prioridade. 


\begin{tabular}{|c|c|l|}
\hline Prioridade & Tipo de Otimização & Estratégia de Otimização \\
\hline \hline Alta & Paralelização Geral & $\begin{array}{l}\text { Para maximar desempenho, } \\
\text { encontre formas de paralelizar } \\
\text { o código sequencial }\end{array}$ \\
\hline Alta & Memória & $\begin{array}{l}\text { Acesso à memória global deve } \\
\text { ser coalesced (4.3.1) }\end{array}$ \\
\hline Alta & Memória & $\begin{array}{l}\text { Minimize utilização de me- } \\
\text { mória global. Utilize memó- } \\
\text { ria compartilhada sempre que } \\
\text { possível (4.3.2) }\end{array}$ \\
\hline Alta & Controle de Fluxo & $\begin{array}{l}\text { Evite caminhos divergentes } \\
\text { dentro de um mesmo warp } \\
\text { (4.4) }\end{array}$ \\
\hline Média & Configuração de Execução & $\begin{array}{l}\text { Número de threads por bloco } \\
\text { deve ser múltiplo de 32 (4.3.3) }\end{array}$ \\
\hline Média & Instruções Aritméticas & $\begin{array}{l}\text { Utilize versões de baixo nível } \\
\text { da biblioteca de matemática } \\
\text { sempre que precisão não for } \\
\text { uma prioridade (4.6.2) }\end{array}$ \\
\hline Baixa & Instruções Aritméticas & $\begin{array}{l}\text { Utilize operadores de shift no } \\
\text { lugar de operações de divisão } \\
\text { ou módulo (4.6.2) }\end{array}$ \\
\hline Baixa & Instruções Aritméticas & $\begin{array}{l}\text { Evite conversões automáticas } \\
\text { de tipo de variáveis (4.6.2) }\end{array}$ \\
\hline
\end{tabular}

Tabela 4.1: Sumário de estratégias de otimização de desempenho 


\section{Capítulo 5}

\section{Geração de Números Aleatórios em GPU}

Em geral, simulações estocásticas são sensíveis à fonte de aleatoriedade, que caracteriza a qualidade estatística de seus resultados. Dessa forma, são necessários geradores de números aleatórios (RNGs) de alta confiança para alimentar tais aplicações. Desenvolvimentos recentes utilizam GPUs de propósito geral (GPGPUs) para acelerá-las. Esse hardware oferece novas possibilidades de paralelização, mas as mesmas causam não somente a reescrita dos algoritmos de simulação existentes como, também, mudanças nas ferramentas que os mesmos utilizam. Como RNGs são a base de qualquer simulação estocástica eles necessitam ser portados para GPGPU.

A seleção de um RNG não é simples. Contudo, há trabalhos que os estudam em uma arquitetura sequencial, como (Knuth, 1997) e (L'Ecuyer, 2007). Em um contexto paralelo, a qualidade estatística é um requisito necessário, mas não suficiente para selecionar um RNG, pois os streams paralelos devem ser independentes. Assim, fornecer um gerador de números aleatórios de alta qualidade é mais difícil em arquiteturas paralelas. Com isso, precisamos levar em conta a técnica de paralelização ao determinar como os streams aleatórios podem ser particionados dentre os elementos paralelos (threads ou processadores) com a garantia de independência entre os mesmos para evitar resultados enviesados nas simulações.

Nas próximas seções apresentaremos um estudo dos principais RNGs disponíveis, bem como sua portabilidade para GPU. Na seção 5.2, apresentamos as principais técnicas de paralelização desses geradores e, baseado no trabalho de (Nguyen, 2007), finalizamos o capítulo com um estudo de otimização em GPU dos geradores de números aleatórios MRG32k3a e Sobol.

\subsection{RNGs}

Geradores de números aleatórios podem ser classificados em três grandes grupos de acordo com sua fonte de aleatoriedade:

- TRNGs (True random number generators): utilizam uma fonte de aleatoriedade física para prover números verdadeiramente imprevisíveis. TRNGs são utilizados principalmente para criptografia. Eles são, em geral, muito lentos para simulação.

- QRNGs (Quasirandom number generators): visam a preencher um espaço n-dimensional com pontos de forma mais uniforme possível.

- PRNGs (Pseudorandom number generators): simulam TRNGs, mas podem ser implementados em software determinístico, tendo seu estado e função de transição previsíveis.

Há dois requisitos básicos desejáveis para PRNGs:

- Longo Período. Todo gerador determinístico eventualmente entra em ciclo. O objetivo, então, é ter o período do ciclo o maior possível. 
- Boa Qualidade Estatística. É desejável que a saída de um PRNG seja praticamente indistinguível em relação a um TRNG de mesma distribuição. Além disso, também não devem exibir correlações ou padrões de geração.

Há inúmeros testes para verificar tais requerimentos de qualidade em algoritmos de arquitetura serial (Knuth, 1997), (L’Ecuyer, 2006) e (Marsaglia, 1995).

\subsubsection{Algoritmos Sequenciais de PRNGs}

Em geral, um gerador de números pseudo-aleatórios consiste em um conjunto finito de estados e uma função de transição $f$ que leva o PRNG de um estado $S$ para um próximo estado $S^{\prime}$,

$$
S^{\prime}=f(S)
$$

a partir de um estado inicial chamado semente $S_{0}$. Dado um estado $S_{n}$ do PRNG, há uma função $g$ que retorna o correspondente número aleatório $x_{n}$ :

$$
x_{n}=g\left(S_{n}\right) \text {. }
$$

A memória utilizada para armazenar o estado de um PRNG é finita. Portanto, o espaço dos estados é finito. Dessa forma, após suficientes passos, tal gerador fecha um ciclo. O tamanho desse ciclo é chamado de período do PRNG.

\section{Linear Congruential Generator}

Linear Congruential Generator (LCG) (Knuth, 1997) é um gerador clássico de PRNGs que possui uma função de transição da forma

$$
x_{n+1}=\left(a x_{n}+c\right) \bmod m .
$$

Assim, o período máximo desse gerador é $m$. Com isso, por exemplo, em uma representação de inteiro de 32-bits, o período pode ser no máximo $2^{32}$. Isso é pouco para simulações estocásticas modernas.

\section{Multiple Recursive Generator}

Multiple Recursive Generator (MRG) é um algoritmo derivado do LCG, que, de modo aditivo, combina dois ou mais geradores. Se $n$ geradores com períodos primos entre si $m_{1}, m_{2}, \ldots, m_{n}$ são combinados, então o período resultante é $\mathrm{LCG}\left(m_{1}, m_{2}, \ldots, m_{n}\right)$. Assim, o período pode ser, no máximo, $m_{1} x, m_{2} x, \ldots, m_{n} x$. Em geral, esses geradores têm boa qualidade estatística e longos períodos. Contudo, os mesmos podem ter muitas multiplicações e divisões o que pode causar perda de desempenho em GPUs.

\section{Lagged Fibonacci Generator}

O Lagged Fibonacci Generator (Knuth, 1997) é comumente utilizado em simulações de Monte Carlo distribuídas. Ele é similar ao LCG, mas introduz um atraso na dependência de estados na função de transição:

$$
x_{n+1}=x_{n} \otimes x_{n-k} \bmod m
$$


onde $\otimes$ é, tipicamente, um operador de multiplicação ou adição. Apesar da simplicidade e pequeno número de variáveis envolvidas, esse gerador necessita que a constante $k$ seja muito grande. Consequentemente, muitas palavras são necessárias para manter o estado e, assim, eventualmente seria necessário utilizar-se de memória global da GPU para transição dos estados, o que implicaria em perda de desempenho.

\section{Mersenne Twister}

Mersenne Twister (Matsumoto e Nishimura, 1998) é um dos geradores mais respeitados. Ele tem período $2^{19.937}$ e excelente qualidade estatística. Entretanto, apresenta um problema similar ao Lagged Fibonacci, pois possui um grande estado que precisa ser atualizado serialmente.

\subsubsection{Algoritmos Sequenciais de QRNGs}

Logo após a introdução do método de Monte Carlo no final dos anos 1940, pesquisadores se interessaram pela possibilidade de substituir amostragens aleatórias em simulações por séries determinísticas. Essa é a ideia fundamental dos QRNGs. Essas sequências são conhecidas como sequências de baixa discrepância. O conceito de baixa discrepância diz respeito à diferença relativa entre séries originadas por sequências determinísticas e aquelas obtidas por variáveis aleatórias uniformes. Assim, essas sequências tem a propriedade de uniformidade dentro de um intervalo de domínio.

Nessa seção, apresentamos algoritmos clássicos para geração de sequências de baixa discrepância (SBD) ou QRNGs. A sequência de Van Der Corput é a mais simples e serve como base na construção de QRNGs em finanças. As sequências mais conhecidas são a de Halton, Faure e, principalmente, Sobol. O princípio de construção dessas sequencias é dividir um hipercubo unitário em hipercubos menores cujas faces são paralelas às faces do hipercubo original. Cada ponto da sequência gerada é colocado em cada hipercubo menor e, assim, a sequência é gerada sucessivamente. O grande desafio na construção de boas SBDs é evitar a aglomeração de pontos em regiões específicas, o que é indesejável para um QRNG.

\section{Sequência de Van Der Corput}

Suponha que desejamos criar uma sequência de baixa discrepância de dezesseis números dentro do intervalo $[0,1)$. Para isso, poderíamos simplesmente escrever a sequência $0,1 / 16,2 / 16, \ldots, 15 / 16$. Essa solução trivial é ruim, pois no meio da sequência já negligenciamos toda a outra metade do intervalo. É preferível uma geração uniforme independente do tamanho da sequência gerada até um dado momento. Para isso, Van Der Corput propôs um algoritmo similar que transforma o número em uma base $b$ e, a cada passo, inverte seus bits (e.g. $001_{2}$ se torna $100_{2}$ ). Isso garante que o bit mais significativo sempre é alternado a cada número gerado. Assim, se o $i$-ésimo número da sequência pertence ao intervalo $[0,1 / 2]$, então o $(i+1)$-ésimo pertencerá ao intervalo $[1 / 2,1]$. A seguir, apresentamos o Algoritmo 1 de Van Der Corput, que encontra o $n$-ésimo número da sequência gerada na base $b$. Um exemplo de execução desse algoritmo para uma sequência na base binária é fornecido na figura 5.1.

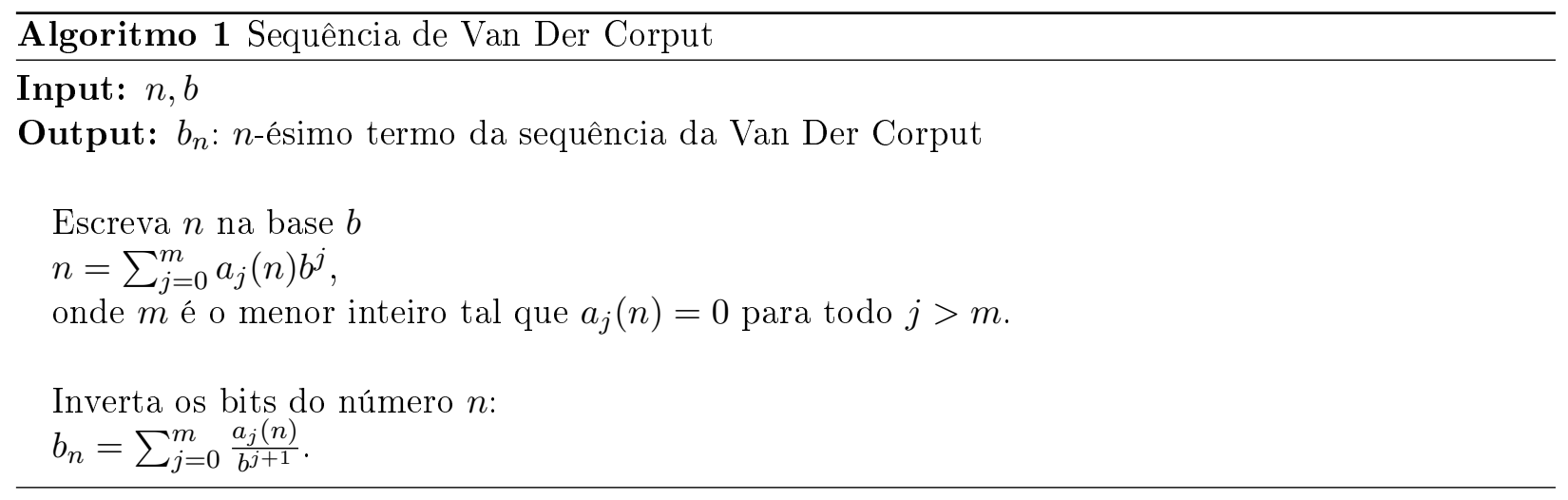




\begin{tabular}{|c|c|c|c|c|c|}
\hline$\imath$ & $\begin{array}{l}\text { Sequência } \\
\text { Trivial }\end{array}$ & Binário & $\begin{array}{l}\text { Binário } \\
\text { Invertido }\end{array}$ & $\begin{array}{l}\text { Van Der } \\
\text { Corput }\end{array}$ & Pontos no intervalo $[0,1]$ \\
\hline 1 & 0 & .0000 & .0000 & 0 & \\
\hline 2 & $1 / 16$ & .0001 & .1000 & $1 / 2$ & \\
\hline 3 & $1 / 8$ & .0010 & .0100 & $1 / 4$ & 0 \\
\hline 4 & $3 / 16$ & .0011 & .1100 & $3 / 4$ & 0 \\
\hline 5 & $1 / 4$ & .0100 & .0010 & $1 / 8$ & $0-0-0-0-0$ \\
\hline 6 & $5 / 16$ & .0101 & .1010 & $5 / 8$ & 0 \\
\hline 7 & $3 / 8$ & .0110 & .0110 & $3 / 8$ & $0-0-0-0-0-0-0$ \\
\hline 8 & $7 / 16$ & .0111 & .1110 & $7 / 8$ & $0-0-0-0-0-0-0-0-0$ \\
\hline 9 & $1 / 2$ & .1000 & .0001 & $1 / 16$ & $\infty+0-0-0-0-0-0-0-0$ \\
\hline 10 & $9 / 16$ & .1001 & .1001 & $9 / 16$ & $\infty+\infty-0-0-\infty, 0-0-0-0$ \\
\hline 11 & $5 / 8$ & .1010 & .0101 & $5 / 16$ & $\infty+\infty-\infty, 0-\infty, 0-0-0-0$ \\
\hline 12 & $11 / 16$ & .1011 & 1101 & $13 / 16$ & $\infty$ \\
\hline 13 & $3 / 4$ & .1100 & 0011 & $3 / 16$ & - \\
\hline 14 & $13 / 16$ & .1101 & 1011 & $11 / 16$ & - \\
\hline 15 & $7 / 8$ & .1110 & .0111 & $7 / 16$ & $000000000000000-0$ \\
\hline 16 & $15 / 16$ & .1111 & .1111 & $15 / 16$ & 00000000000000000 \\
\hline
\end{tabular}

Figura 5.1: Sequência de Van Der Corput em base binária

\section{Sequência de Halton}

A sequência de Halton é uma extensão multi-dimensional da sequência de Van Der Corput. Assim, cada dimensão em uma sequência de Halton corresponde a uma sequência de Van Der Corput construída em uma base diferente. Mais especificamente, a $i$-ésima dimensão de Halton corresponde à sequência de Van Der Corput na base do $i$-ésimo número primo ordenado crescentemente. A tabela 5.1 exibe os primeiros termos dessa sequência. Na figura 5.2 podemos observar mil pontos

\begin{tabular}{c|c|c|c|c}
\hline Termo & Dimensão 1 & Dimensão 2 & Dimensão 3 & Dimensão 4 \\
\hline \hline & Base 2 & Base 3 & Base 5 & Base 7 \\
\hline \hline 2 & $1 / 2$ & $1 / 3$ & $1 / 5$ & $1 / 7$ \\
\hline 3 & $1 / 4$ & $2 / 3$ & $2 / 5$ & $2 / 7$ \\
\hline 4 & $3 / 4$ & $1 / 9$ & $3 / 5$ & $3 / 7$ \\
\hline 5 & $1 / 8$ & $4 / 9$ & $4 / 5$ & $4 / 7$ \\
\hline
\end{tabular}

Tabela 5.1: Sequência de Halton

gerados pela função padrão de Matlab para geração de números aleatórios uniformes. Na figura 5.3 são exibidos números bi-dimensionais gerados pela sequência de Halton. É fácil notar, visualmente, que a Sequência de Halton resultou em uma amostra mais uniformemente distribuída no quadrado unitário. 


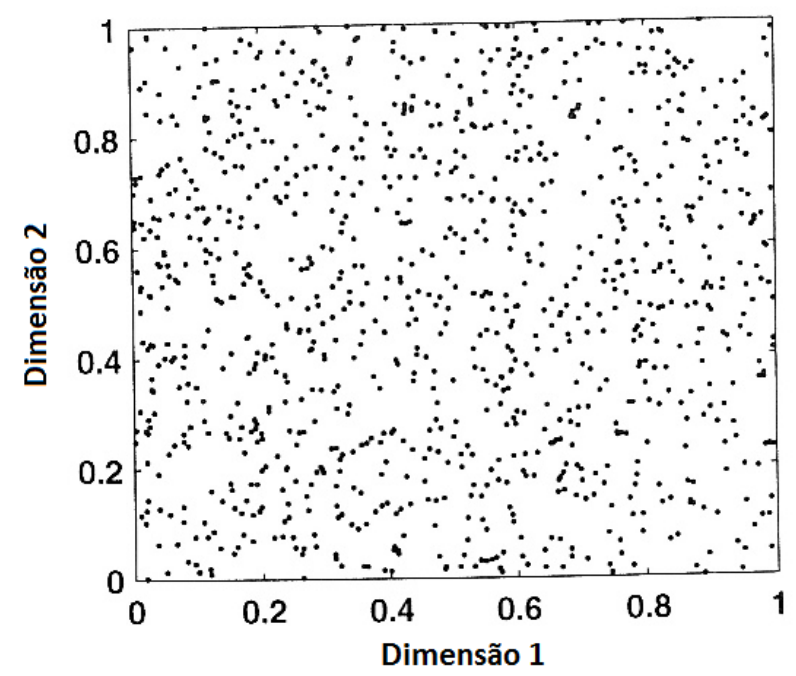

Figura 5.2: Função Matlab para geração de números aleatórios uniformes (Huynh et al., 2011)

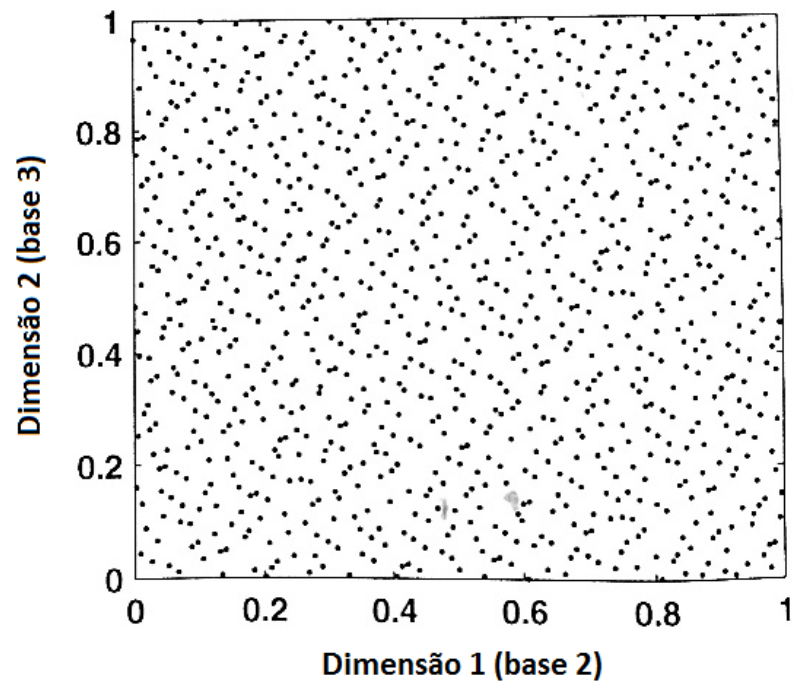

Figura 5.3: Sequência de Halton bi-dimensional (Huynh et al., 2011)

\section{Sequência de Faure}

A sequência de Faure é parecida com a sequência de Halton. Ela também utiliza a sequência de Van Der Corput de forma multidimensional. Contudo, Faure utiliza a mesma base para todas as dimensões. Além disso, os $n$-ésimos termos gerados são resultado de uma permutação dos $(n-1)$ ésimos termos gerados, conforme recursão:

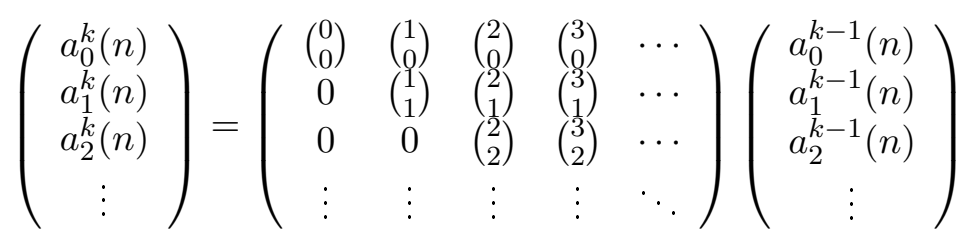

\subsubsection{Distribuições não Uniformes}

Apesar dos geradores uniformes apresentados na seção 5.1.1 formarem a base para a maioria dos procedimentos estocásticos, outras sequências de distribuição não uniforme podem ser construídas 
a partir dessas.

\section{Método de Inversão}

O método mais simples de geração de distribuições não uniformes é por inversão direta da função de distribuição, quando possível. Suponha que desejamos gerar uma sequência distribuída de acordo com a função de densidade $p(x)$, onde $x$ é um escalar real. A distribuição de probabilidade acumulada é

$$
P[x \leq z]=P(z)=\int_{-\infty}^{z} p(t) d t .
$$

Se for possível obter $P^{-1}$, então podemos gerar uma sequência de números distribuídos não uniformemente e mutuamente independentes como

$$
x_{i}=P^{-1}\left(u_{i}\right),
$$

onde $u_{1}, u_{2}, \ldots$ é uma sequência de números uniformemente distribuídos e mutuamente independentes de números reais pertencentes ao intervalo $(0,1)$.

\section{Método de Aceitação e Rejeição}

Suponha que conheçamos um método para simular uma variável aleatória $Y$ com função de densidade de probabilidade $g(x)$. O método de aceitação e rejeição utiliza essa função como base para simular a distribuição de $X$ que tem como função de densidade de probabilidade $f(x)$. Para isso, devemos simular $Y$ e aceitar esse valor com uma probabilidade proporcional a $f(Y) / g(Y)$.

Assim, seja $c$ uma constante tal que

$$
\frac{f(y)}{g(y)} \leq c, \quad \forall y .
$$

Deve-se simular $Y$ tantas vezes quanto se queira e devemos aceitar o valor dessa variável aleatória, em cada simulação, como uma amostra para $X$ se

$$
U \leq \frac{f(Y)}{c g(Y)}
$$

onde $U \sim U[0,1]$.

\section{Transformada Gaussiana}

A distribuição Gaussiana é um importante exemplo de distribuição não uniforme. Por isso, há diversas formas de transformação de uma distribuição uniforme para uma distribuição normal, como o método de Ziggurat (Marsaglia e Tsang, 2000) e o método Polar (Miller et al., 2010).

Como GPUs são sensíveis a laços e desvios de fluxo, o método de Box-Muller (Box e Muller, 1958) é uma excelente escolha para transformação Gaussiana. Nesse método, duas amostras uniformes $u_{0}$ e $u_{1}$ pertencentes ao intervalo $(0,1)$ são tomadas e em seguida transformadas em duas amostras normais $n_{0}$ e $n_{1}$ por meio da relação:

$$
\begin{aligned}
& n_{0}=\sin \left(2 \pi u_{0}\right) \sqrt{-2 \log \left(u_{1}\right)} \\
& n_{1}=\cos \left(2 \pi u_{0}\right) \sqrt{-2 \log \left(u_{1}\right)}
\end{aligned}
$$

Em (Lee et al., 2009) foi demonstrado um speedup de 170 vezes no cálculo do método de BoxMuller em GPU.

Para uma comparação detalhada de diferentes técnicas para geração de variáveis aleatórias normais veja (Roy, 2002). 


\subsection{Técnicas de Paralelização de PRNGs}

Para gerar números aleatórios em um computador paralelo podemos nos basear em um RNG serial que distribui sua sequência gerada sequencialmente ao longo dos processadores disponíveis. Uma maneira mais moderna seria parametrizar o RNG de modo diferente de acordo com o processador escolhido. Com esse objetivo, há diversas técnicas de obtenção de streams paralelos de números aleatórios que exploram a divisão do ciclo do RNG em saltos na transição de estados ou mesmo no particionamento da sequência principal em um dado gerador de sub-sequencias. A seguir, apresentamos algumas das principais técnicas para esse fim.

\subsubsection{Central Server}

A técnica Central Server consiste em um servidor central rodando um RNG provendo números pseudo-aleatórios por demanda para diferentes processadores lógicos (PL). Algumas desvantagens de essa técnica são: simulações nessa abordagem são de difícil reprodução e o servidor central pode se tornar um gargalo ao se considerar muitos PLs.

\subsubsection{Sequence Splitting}

Também conhecido como Regular Spacing, a técnica Sequence Splitting consiste em dividir de modo determinístico uma sequência de estados em blocos contíguos sem sobreposição. Dada uma sequência $S_{i}, i=0,1, \ldots$ e $N$ streams, o $j$-ésimo stream contem a sequência $S_{p+(j-1) m}, p=0,1, \ldots$, onde $m$ é o tamanho do stream. Assim, se a sequência original é

$$
S_{0}, S_{1}, S_{2}, \ldots, S_{N-1}, S_{N}, \ldots, S_{2 N-1}, S_{2 N}
$$

então a sequência gerada para o stream 0 é

$$
S_{0}, S_{1}, S_{2}, \ldots, S_{N-1}
$$

Apesar de geradores sequenciais com correlações de grande intervalo serem comuns, tal particionamento contíguo pode levar a correlação em intervalos menores (Matteis e Pagnutti, 1990).

\subsubsection{Random Spacing}

Random Spacing constrói partições de $N$ streams ao inicializar o mesmo gerador com $N$ estados aleatórios. Dessa forma, as sementes do gerador são produzidas por outro RNG. Essa técnica é interessante quando o período dos geradores é muito grande, o que diminui a probabilidade de sobreposição nos streams. Apesar dessa técnica ser de fácil uso, sempre há o risco de má inicialização das sementes. Em 2009, (Reuillon et al., 2008) propuseram um milhão de estados para o Mersenne Twister e demonstraram uma perda de qualidade estatística quando há um número desbalanceado de 0 s e $1 \mathrm{~s}$ nos estados iniciais binários do algoritmo.

\subsubsection{Leap Frog}

Dados $n$ streams, cada stream na técnica Leap Frog seleciona números que estão $n$ posições distantes entre si em relação a sequência original gerada. Assim, dada uma sequência $S_{i}, i=0,1, \ldots$ e $N$ streams, o $j$-ésimo stream contem a sequência $S_{p N+(j-1)}, p=0,1, \ldots$, onde $p$ é o período da sequência original e $p / N$ o período de cada stream. Dessa forma, se a sequência original é

$$
S_{0}, S_{1}, S_{2}, \ldots, S_{N-1}, S_{N}, \ldots, S_{2 N-1}, S_{2 N}
$$

então a sequência gerada para o stream 0 é dado por:

$$
S_{0}, S_{N-1}, S_{2 N-1}, \ldots
$$


A geração de números aleatórios em saltos como esse é muitas vezes referenciado como uma técnica de skip-ahead.

\subsection{Multiple Recursive Generator MRG32k3a em GPU}

\subsubsection{Formulação}

L'Ecuyer estudou CMRGs (Combined Multiple Recursive Generators) visando a um gerador que tivesse boas propriedades aleatórias e uma implementação simples. O mais conhecido CMRG é o MRG32k3a (Fischer et al., 1999), que é definido por

$$
\begin{aligned}
y_{1, n}=\left(a_{12} y_{1, n-2}+a_{13} y_{1, n-3}\right) & \bmod m_{1}, \\
y_{2, n}=\left(a_{21} y_{2, n-1}+a_{23} y_{2, n-3}\right) & \bmod m_{2}, \\
x_{n}=\left(y_{1, n}+y_{2, n}\right) & \bmod m_{1},
\end{aligned}
$$

para todo $n \geq 3$, onde

$$
\begin{aligned}
& a_{1,2}=14403580, \quad a_{1,3}=-810728, \quad m_{1}=2^{32}-209 \\
& a_{2,1}=527612, \quad a_{2,3}=-1370589, \quad m_{2}=2^{32}-22853
\end{aligned}
$$

e $x_{n}$ representa a $n$-ésima saída do gerador. Dessa forma, o estado do gerador é definido pelo par de vetores

$$
Y_{i, n}=\left(\begin{array}{l}
y_{i, n} \\
y_{i, n-1} \\
y_{i, n-2}
\end{array}\right)
$$

para $i=1,2$. Com isso, a recorrência da equação 5.1 pode ser expressa como

$$
Y_{i, n}=A_{i} Y_{i, n} \bmod m_{i},
$$

onde $A_{i}$ é uma matriz de constantes e, portanto,

$$
Y_{i, n+p}=A_{i}^{p} Y_{i, n} \bmod m_{i}
$$

para um parâmetro $p$ constante.

\subsubsection{Paralelização}

A equação 5.2 é uma forma eficiente de transição arbitrária entre estados do MRG. Assim, a técnica Sequence Splitting poderia ser utilizada apropriadamente para paralelização de tal gerador. Nessa estratégia, cada thread tem uma cópia do estado e produz um segmento contíguo da sequência MRG32k3a independente de todas as outras threads.

Para um cálculo eficiente do produtório matricial $A_{i}^{p}$ com grandes valores de $p$, pode-se utilizar uma estratégia de "Divisão e Conquista" de elevar ao quadrado iterativamente a matriz $A_{i}$ (Knuth, 1997). A começar pela decomposição em fatores binários do expoente

$$
p=\sum_{j=0}^{k} g_{j} 2^{j},
$$

onde $g_{j} \in\{0,1\}$, calcula-se a sequência

$$
A_{i}, A_{i}^{2}, A_{i}^{4}, A_{i}^{8}, A_{i}^{16}, \ldots, A_{i}^{2^{k}}, \bmod m_{i}
$$

Com isso, chegamos a 


$$
A_{i}^{p} Y_{i}=\prod_{j=0}^{k} A^{g_{j} 2^{j}} \bmod m_{i},
$$

Dessa forma, o cálculo pode ser efetuado em $O\left(\log _{2} p\right)$ passos em vez de $O(p)$, como no algoritmo original. Tal procedimento pode ser generalizado para uma decomposição de $p$ em uma base $b$ qualquer:

$$
p=\sum_{j=0}^{k} g_{j} b^{j},
$$

com $g_{j} \in\{0,1, \ldots, b-1\}$. Uma base maior aumentaria a velocidade do procedimento ao custo de mais memória para armazenar as potências de $A_{i}$ na expansão de $p$.

\subsubsection{Implementação}

Primeiramente, é necessário armazenar em memória de host o conjunto de matrizes $A_{i}^{g_{j} b^{k}}$ :

$$
\begin{array}{cccc}
A_{i} & A_{i}^{2} & \cdots & A_{i}^{b-1} \\
A_{i}^{b} & A_{i}^{2 b} & \cdots & A_{i}^{(b-1) b} \\
\vdots & \vdots & \cdots & \vdots \\
A_{i}^{b^{k}} & A_{i}^{2 b^{k}} & \cdots & A_{i}^{(b-1) b^{k}}
\end{array}
$$

Como um exemplo, podemos tomar $b=8$ e $k=20$. Como o estado é pequeno, aproximadamente 10KB de memória, toda a matriz de potências pode ser copiada para a memória constante da GPU, o que provê grande aumento de desempenho.

Para gerar um total de $N$ números aleatórios, um kernel pode ser configurado com $T$ threads no total, e a $i$-ésima thread com $1 \leq i \leq T$ deve avançar seu estado em $(i-1) N / T$ posições e gerar $p=N / T$ números. Assim, o $j$-ésimo número aleatório gerado seria indexado por

$$
\text { prngID }=j+p * \text { threadId } x . x+p * \text { blockIdx. } x * \text { blockDim. } x .
$$

Note que, como cada thread gera um segmento contíguo de números aleatórios, tal armazenamento em memória global não é coalesced. Coalesced memory pode ser obtida indexando o j-ésimo número aleatório por

$$
\text { prngID }=\text { threadId } x . x+j * \text { blockDim. } x+p * \text { blockId } x . x * \text { blockDim. } x
$$

\subsection{Sobol em GPU}

Sobol (Sobol, 1967) propôs sua sequência como um método alternativo para integração numérica em um hipercubo unitário. A ideia é construir uma sequência que preenche um cubo de maneira regular. Então, a integral é aproximada como uma média dos valores da função desses pontos. Essa abordagem é eficiente em grandes dimensões onde métodos tradicionais de quadratura numérica são custosos.

\subsubsection{Formulação}

Para gerar o $j$-ésimo componente de pontos de uma sequência de Sobol, escolhemos um polinômio primitivo de um grau $s_{j}$ no corpo $\mathbb{Z}_{2}$,

$$
x^{s_{j}}+a_{1, j} x^{s_{j}-1}+a_{2, j} x^{s_{j}-2}+\ldots+a_{s_{j}-1, j} x+1
$$


onde os coeficientes $a_{1, j}, a_{2, j}, \ldots, a_{s_{j}-1, j} \in\{0,1\}$. Definimos uma sequência de inteiros positivos $\left\{m_{1, j}, m_{2, j}, \ldots\right\}$ pela relação de recorrência

$$
m_{k, j}=2 a_{1, j} m_{k-1, j} \oplus 2^{2} a_{2, j} m_{k-2, j} \oplus \ldots \oplus 2^{s_{j}-1} a_{s_{j}-1, j} m_{k-s_{j}+1, j}
$$

onde $\oplus$ denota o operador OR exclusivo bit-a-bit. Os valores iniciais $m_{1, j}, m_{2, j}, \ldots$ podem ser escolhidos livremente contanto que cada $m_{k, j}, 1 \leq k \leq s_{j}$, seja ímpar e menor que $2^{k}$. As chamadas direções da sequência $\left\{v_{1, j}, v_{2, j}, \ldots\right\}$ são definidas por

$$
v_{k, j}=\frac{m_{k, j}}{2^{k}}
$$

Assim, o $j$-ésimo componente do $i$-ésimo ponto de uma sequência de Sobol é dado por

$$
x_{i, j}=i_{1} v_{1, j} \oplus i_{2} v_{2, j} \oplus \ldots
$$

onde $i$ tem sua representação binária dada por $i=\left(\ldots i_{3} i_{2} i_{1}\right)_{2}$.

Para obter de sequências multidimensionais de Sobol, tomamos direções diferentes para cada dimensão. Porém, é preciso cuidado, pois escolhas ruins nas direções levam à não uniformidade na distribuição dos pontos da sequência.

\subsubsection{Paralelização}

Utilizando código de Gray, Antonov e Saleev (Antonov e Saleev, 1979) encontraram uma forma simples de obter o $(i+1)$-ésimo elemento de uma sequência de Sobol a partir do $i$-ésimo elemento. Eles mostraram que

$$
\begin{aligned}
x_{n} & =g_{1} m_{1} \oplus g_{2} m_{2} \oplus \ldots \\
& =x_{n-1} \oplus m_{f(n-1)}
\end{aligned}
$$

onde, o código de Gray de $n$ é dado por $n \oplus(n / 2)=\ldots g_{3} g_{2} g_{1}$ e $f(n)$ retorna o índice do bit zero mais a direita na expansão binária de $n$.

Para geração de até $2^{k}$ pontos é necessário um conjunto de $k$ números de direção. Assim, para obtenção do elemento $x_{n}$ a partir do elemento $x_{n-1}$ seriam necessárias, no máximo, $k$ iterações, onde cada iteração faria uma inversão de bit e, possivelmente, uma operação XOR. Dessa forma, podemos concluir que a equação 5.5 fornece um modo eficiente de implementação de uma estratégia do tipo skip-ahead, já que é possível realizar saltos sem muito custo. Portanto, o algoritmo poderia ser paralelizado tal qual na paralelização do MRG32k3a apresentada, onde cada thread gera um bloco de pontos e depois realiza um skip-ahead.

Ao adicionarmos $2^{p}$ em $n=\left(\ldots b_{3} b_{2} b_{1}\right)_{2}$, podemos notar que os $p$ primeiros bits de $n$ se mantem inalterados: adicionar 1 ao número $n$ em $2^{p}$ vezes resulta em alternar $b_{1}$ em $2^{p}$ vezes, $b_{2}$ em $2^{p-1}$ vezes e, assim, por diante. Considere, agora, o comportamento da função $f(n+i)$ para $1 \leq i \leq 2^{p}$ : como estamos enumerando todas as permutações dos primeiros $p$ bits de $n$, então, $f(n+i)=1 \mathrm{em}$ $2^{p-1}$ vezes, $f(n+i)=2$ em $2^{p-2}$ vezes e assim, por diante. Portanto, $f(n+i)=j$ em $2^{p-j}$ vezes, para $j \in\{1,2, \ldots, p\}$. Note que, $f(n+1)$ retornará um índice $q$ maior do que $p$ apenas uma vez, já que os primeiros $p$ bits valem todos 1 uma única vez. Sabendo que duas operações de XOR se cancelam, podemos reescrever a equação 5.5 como:

$$
\begin{aligned}
x_{n+2^{p}} & =x_{n} \oplus \overbrace{m_{1} \oplus \ldots \oplus m_{1}}^{2^{p-1} \text { vezes }} \oplus \overbrace{m_{2} \oplus \ldots \oplus m_{2}}^{2^{p-2} \text { vezes }} \oplus \ldots \oplus m_{p} \oplus m_{q} \\
& =x_{n} \oplus m_{p} \oplus m_{q}
\end{aligned}
$$

Assim, podemos utilizar a equação 5.7 em uma estratégia eficiente do tipo leapfrog, na qual cada stream possui um espaçamento de tamanho $2^{p}$. 


\subsubsection{Implementação}

As direções $m_{i}$ podem ser pré-computadas no host e copiadas para o device. Em uma sequência de Sobol de 32-bits temos no máximo 32 valores de $m_{i}$. Como as dimensões de uma sequência D-dimensional de Sobol são independentes, podemos utilizar um bloco para cada dimensão. Assim, para cada dimensão, cada bloco deve lançar $2^{p}$ threads e os 32 valores de $m_{i}$ podem ser armazenados na memória compartilhada, que é mais veloz. Dentro do bloco, a $i$-ésima thread deve computar $x_{i}$ pela equação original 5.5 e, então, deve fazer o skip-ahead dado pela equação 5.7. Note que threads sucessivas geram números de Sobol sucessivos, logo obtemos Coalesced Memory.

\subsection{Bibliotecas para RNGs em GPU}

\subsubsection{NVIDIA CURAND}

Introduzido na versão CUDA 3.2, a biblioteca CURAND foi projetada para gerar números aleatórios de modo simples em GPGPUs com suporte a CUDA. Ela gera números quasi-aleatórios e pseudo-aleatórios tanto em GPGPU ou CPU. Exceto pela fase de inicialização do gerador, a API é praticamente a mesma em ambas plataformas.

Em uma versão mais recente, com CUDA SDK 4.0, CURAND vem com uma implementação de Sobol para geração de números quasi-aleatórios e XORWOW (Marsaglia, 2003) para números pseudo-aleatórios. Ambos podem apresentar uma aceleração de oito vezes em relação à biblioteca MKL da Intel (NVIDIA, 2011). O algoritmo PRNG escolhido pela NVIDIA é conhecido pela sua velocidade e pelo baixo uso de memória.

\subsubsection{Thrust::random}

Thrust::random faz parte de uma biblioteca de propósito geral disponível para GPGPU chamada Thrust. Esse projeto de código aberto tem a intenção de prover uma biblioteca para GPGPU equivalente a bibliotecas clássicas de $\mathrm{C}++$ como STL ou Boost. Classes são divididas em diversos namespaces, dos quais Thrust::random é um exemplo. Essa biblioteca implementa três tipos de PRNGs: Linear Congruential Generator (LCG), Linear Feedback Shift (LFS) e Substract With Carry (SWC).

\subsubsection{ShoveRand}

ShoveRand (C. Mazel e Hill, 2011) é uma proposta de meta-modelo de representação de RNGs em qualquer plataforma. Assim, ele foi desenhado como um arcabouço para que desenvolvedores integrassem suas implementações de RNGs em GPGPU. O modelo apresenta uma hierarquia constituída por quatro classes principais: RNG, Algorithm, ParameterizesStatus e SeedStatus, como pode ser visto na figura 5.4. As duas últimas são agregadas pelas diferentes possibilidades de implementação da classe Algorithm. A classe RNG, associa-se à classe Algorithm e expõe uma interface ao usuário. 


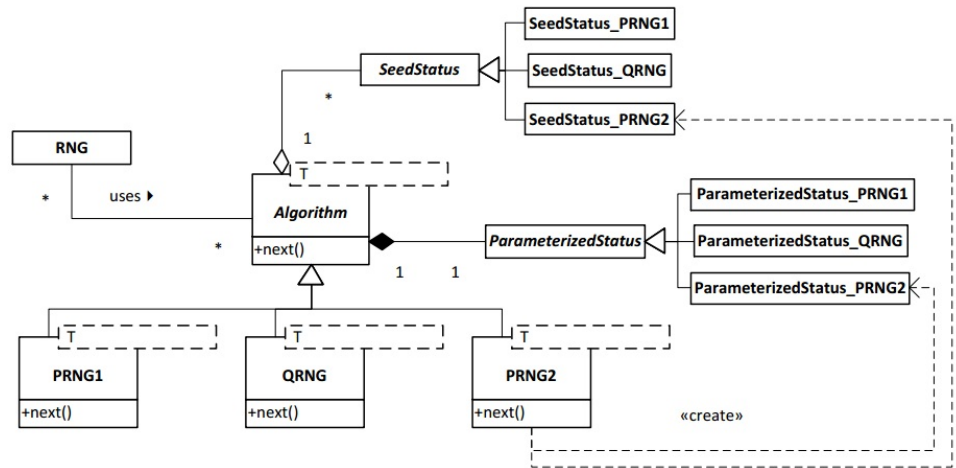

Figura 5.4: ShoveRand meta-modelo (C. Mazel e Hill, 2011) 


\section{Parte III}

\section{Fundamentos da Modelagem Matemática}





\section{Capítulo 6}

\section{Simulação Estocástica}

A presença de incerteza é um fato em modelagem financeira. A menos em problemas de baixa complexidade, essa característica não pode ser ignorada. Assim, a modelagem de sistemas complexos e com características estocásticas vem sendo utilizada cada vez mais. Com isso, este capítulo tem o objetivo de apresentar de forma sucinta e elementar alguns aspectos da área de modelagem estocástica em finanças que serão usados nos casos de estudo do trabalho. A teoria apresentada está diretamente relacionada com aplicações e permite, por meio do método de Monte Carlo, a implementação numérica em problemas práticos.

Assim, na seção 6.1, apresentamos fundamentos de Simulações de Monte Carlo. Em seguida, nas seções 6.2 e 6.3, abordamos processos estocásticos e resolução de Equações Diferenciais Estocásticas. Na seção 6.4, estudamos métodos práticos de solução numérica dessas equações.

\subsection{Fundamentos de Simulações de Monte Carlo}

Em finanças, a precificação de ativos geralmente consiste em computar uma expectativa de ganho. Nesta seção, descrevemos como técnicas de simulação estocástica podem ser utilizadas para aproximar essas esperanças matemáticas.

\subsubsection{Integração de Monte Carlo}

O método de Monte Carlo (MC) é um método utilizado para estimar numericamente funções de variáveis aleatórias. Suas origens remontam à computação de $\pi$ por Laplace e Bouffon, por meio de um experimento aleatório. Mais tarde, durante a construção da bomba atômica em Los Alamos, Von Newman and Ulam aprimoraram essa técnica para calcular integrais complexas.

A integração de Monte Carlo é motivada pela Lei dos Grandes Números: se $X_{i}$ é uma coleção de variáveis aleatórias independentes e identicamente distribuídas de função densidade $q(x)$ e pertencem ao intervalo $[0,1]$, então

$$
\lim _{N \rightarrow \infty} \frac{1}{N} \sum_{i=1}^{N} X_{i}=\int_{0}^{1} x q(x) d x, \quad a . s .
$$

Além disso,

$$
\operatorname{var}\left(\frac{1}{N} \sum_{i=1}^{N} X_{i}\right)=\frac{\sigma_{x}^{2}}{N}
$$

onde $\sigma_{x}^{2}=\operatorname{var}\left(X_{i}\right)$. Se $\sigma_{x}^{2}$ é desconhecido, podemos estimá-lo como

$$
\hat{\sigma}_{x}^{2}=\frac{1}{N-1} \sum_{i=1}^{N}\left(X_{i}-\hat{X}\right)^{2},
$$


onde $\hat{X}$ é a média amostral

$$
\hat{X}=\frac{1}{N} \sum_{i=1}^{N} X_{i} .
$$

Assim, a Lei dos Grandes Números sugere um procedimento de integração numérica, como demonstramos a seguir.

Suponha que desejamos computar $I_{f}=\int_{0}^{1} f(x) d x$. Se $X \sim U[0,1]$, então

$$
\mathbb{E}[f(x)]=\int_{0}^{1} f(x) d x
$$

ou seja, a integral é estimada pela esperança da variável aleatória $Y=f(X)$. Para isso, o método de Monte Carlo gera $N$ amostras $x_{i} \sim U[0,1], i=1, \ldots, N$ e considera

$$
\hat{I}_{f}=\frac{1}{N} \sum_{i=1}^{N} f\left(x_{i}\right)
$$

como uma estimativa de $\int_{0}^{1} f(x) d x$. Dessa forma, essa aproximação é, por sua vez, uma variável aleatória, $\hat{I}_{f}$, com variância

$$
\sigma_{\hat{I}_{f}}^{2}=\frac{1}{N} \int_{0}^{1}\left(f(x)-I_{f}\right)^{2} d x=\frac{\sigma_{f}^{2}}{N}
$$

Como $\sigma_{f}^{2}$ é desconhecido, tomamos $\hat{\sigma}_{f}^{2}$ como estimativa de $\sigma_{f}^{2}$ :

$$
\hat{\sigma}_{f}^{2}=\frac{1}{N-1} \sum_{i=1}^{N}\left(f\left(x_{i}\right)-\hat{I}_{f}\right)^{2} .
$$

Pelo teorema central do limite, podemos afirmar que

$$
\hat{I}_{f} \sim \mathcal{N}\left(\mathbb{E}[f(x)], \frac{1}{N} \operatorname{var}(f(x))\right) .
$$

Assim, o valor estimado $\hat{I}_{f}$ terá um desvio da verdadeira esperança $\mathbb{E}[f(x)]$ na ordem de $1 / \sqrt{N}$. Dado que $P(|Z|<1,96) \approx 0,95, Z \sim \mathcal{N}(0,1)$, podemos construir o seguinte intervalo de confiança a um nível de $95 \%$ para estimativa de $\hat{I}_{f}$ :

$$
\left(\hat{I}_{f}-1,96 \frac{\hat{\sigma}}{\sqrt{N}}, \hat{I}_{f}+1,96 \frac{\hat{\sigma}}{\sqrt{N}}\right) .
$$

\subsubsection{Técnicas de Redução de Variância}

O método tradicional de Monte Carlo é comumente utilizado sem modificações. Apesar de ser um método não enviesado, sua variância pode ser muito grande. Há uma variedade de técnicas que podem reduzir substancialmente a variância da estimativa, enquanto mantém a característica importante de estimador não enviesado do método.

\section{Variáveis Antitéticas}

Para se obter a média de uma variável aleatória com grande variância pode ser necessário um grande número de simulações para atingir a precisão desejada. Em contraste, quando a variância é pequena, o número de simulações exigidas é baixo. Assim, é de grande importância encontrarmos transformações que reduzam a variância do resultado da simulação. 
Definição 1. Dizemos que a variável aleatória $X^{a}$ é antitética à variável aleatória $X$ se correl $\left(X, X^{a}\right)=$ -1 .

A utilização de variáveis antitéticas é umas das técnicas mais simples. Ela gera $N$ variáveis aleatórias independentes e identicamente distribuídas (i.i.d.) $X_{i}$ e constrói $N$ outras variáveis i.i.d. a partir dessas com a mesma distribuição mas com correlações perfeitamente negativas com as primeiras.

Por exemplo, seja $f$ uma função monotonicamente crescente. Se $X \sim U[0,1]$, então $f(x)$ e $f(1-x)$ são negativamente correlacionados. Assim, a ideia da técnica é reduzir a variância de $\hat{I}_{f}$ ao estimar $I_{f}$ com a estimativa antitética

$$
\hat{I}_{f}^{a}=\frac{1}{2 N} \sum_{i=1}^{N}\left(f\left(x_{i}\right)+f\left(1-x_{i}\right)\right) .
$$

Esse estimador antitético é um estimador não enviesado de $I_{f}$, mas possui variância menor que o estimador tradicional dado que os termos do somatório são negativamente correlacionados.

Proposição 1. Considere um conjunto de $2 N$ variáveis aleatórias i.i.d. $\left\{X_{i}\right\}_{i=1}^{2 N}$ e a geração de $N$ variáveis aleatórias i.i.d. $\left\{X_{i}^{a}\right\}_{i=1}^{N}$, tal que, $X_{i}$ é antitética a $X_{i}^{a}$ e $\mathbb{E}\left[X_{i}\right]=\mathbb{E}\left[X_{i}^{a}\right]$. Sejam $\hat{I}_{f}$ e $\hat{I}_{f}^{a}$, respectivamente, os estimadores de média sobre os conjuntos $\left\{X_{i}\right\}_{i=1}^{2 N}$ e $\left\{X_{i}\right\}_{i=1}^{N} \cup\left\{X_{i}^{a}\right\}_{i=1}^{N}$, então $\operatorname{var}\left(\hat{I}_{f}^{a}\right)=\operatorname{var}\left(\hat{I}_{f}\right) / 2$.

Demonstração. Temos que,

$$
\hat{I}_{f}^{a}=\frac{1}{2 N} \sum_{k=1}^{N}\left(X_{k}+X_{k}^{a}\right) .
$$

Seja $\sigma_{X}^{2}$ a variância do estimador sobre o conjunto $\left\{X_{i}\right\}_{i=1}^{N}$, então

$$
\begin{aligned}
\operatorname{var}\left(\hat{I}_{f}^{a}\right) & =\frac{1}{4 N^{2}} \sum_{k=1}^{N} \sigma_{X}^{2}+\frac{1}{4 N^{2}} \sum_{k=1}^{N} \sigma_{X}^{2} \\
& +\frac{1}{4 N^{2}} \sum_{k=1}^{N} \sum_{j=1}^{N} \mathbb{E}\left[\left(X_{k}-\mu_{X}\right)\left(X_{j}^{a}-\mu_{X}\right)\right] .
\end{aligned}
$$

Todas as variáveis $X_{k}$ são independentes duas a duas, bem como as variáveis $X_{k}^{a}$. Assim, todas as $2 N$ variáveis são independentes, exceto pelos pares $X_{k}$ e $X_{k}^{a}$. Dessa forma, a esperança do último termo da equação anterior é zero, exceto quando $k=j$, onde

$$
\mathbb{E}\left[\left(X_{k}-\mu_{X}\right)\left(X_{j}^{a}-\mu_{X}\right)\right]=-\sigma_{X}^{2} .
$$

Assim,

$$
\operatorname{var}\left(\hat{I}_{f}^{a}\right)=\frac{1}{4 N} \sigma_{X}^{2}+\frac{1}{4 N} \sigma_{X}^{2}-\frac{1}{4 N} \sigma_{X}^{2}=\frac{1}{4 N} \sigma_{X}^{2}
$$

Como,

$$
\operatorname{var}\left(\hat{I}_{f}\right)=\frac{1}{2 N} \sigma_{X}^{2}
$$

então,

$$
\operatorname{var}\left(\hat{I}_{f}^{a}\right)=\frac{\operatorname{var}\left(\hat{I}_{f}\right)}{2}
$$


Assim, pela Proposição 1, podemos concluir que é possível reduzir a variância do estimador em até duas vezes utilizando a técnica de variáveis antitéticas.

\section{Variáveis de Controle}

Como visto, a utilização de variáveis antitéticas pode reduzir a variância do estimador utilizado de forma considerável. Essa técnica explora a propriedade de correlação negativa das variáveis. Portanto, se utilizarmos outras variáveis, não necessariamente antitéticas, mas negativamente correlacionadas com as variáveis geradas, é possível reduzir a variância do estimador.

Considere uma variável aleatória $X$ e outra variável aleatória $Y$ correlacionada com $X$. Chamamos de $Y$, variável de controle ${ }^{1}$ de $X$. Assumindo que a média de $Y$ é conhecida, podemos construir a seguinte nova variável:

$$
X^{*}=X+\alpha(Y-\mathbb{E}[Y]) .
$$

Podemos observar que a média de $X^{*}$ é idêntica à de $X: m_{X}=m_{X}^{*}$. Assim, podemos estimar a média de $X$ pela estimativa de média de $X^{*}$.

Proposição 2. Se $Y$ é uma variável de controle de $X$,

$$
X^{*}=X+\alpha(Y-\mathbb{E}[Y]),
$$

então, $\exists \alpha \in \mathbb{R}: \operatorname{Var}\left(X^{*}\right) \leq \operatorname{Var}(X)$.

Demonstração. Temos que a variância de $X^{*}$ é

$$
\begin{aligned}
\operatorname{Var}\left(X^{*}\right) & =\mathbb{E}\left[\left(X^{*}-m_{X^{*}}\right)^{2}\right]=\mathbb{E}\left[\left(X+\alpha(Y-\mathbb{E}[Y])-m_{X^{*}}\right)^{2}\right] \\
& =\operatorname{Var}(X)+\alpha^{2} \operatorname{Var}(Y)+2 \alpha \operatorname{Cov}(X, Y) .
\end{aligned}
$$

Deseja-se que a variância seja mínima, então devemos escolher $\alpha=\alpha^{*}$ tal que

$$
\frac{d \operatorname{Var}\left(X^{*}\right)}{d \alpha}=0 .
$$

Isso implica que

$$
\alpha^{*}=-\frac{\operatorname{Cov}(X, Y)}{\operatorname{Var}(Y)}
$$

Com essa escolha de $\alpha$ temos que a variância de $X^{*}$ se torna

$$
\operatorname{Var}\left(X^{*}\right)=\operatorname{Var}(X)-\frac{|\operatorname{Cov}(X, Y)|^{2}}{\operatorname{Var}(Y)}
$$

Isso mostra que $\operatorname{Var}\left(X^{*}\right) \leq \operatorname{Var}(X)$

\section{Amostragem Estratificada}

A técnica de Amostragem Estratificada ${ }^{2}$ baseia-se na observação de que a variância de uma função $f(x)$ sobre um sub-intervalo de seu domínio $x \in[0,1]$ é comumente menor do que sobre o intervalo inteiro. A partir dessa divisão, é possível realizar mais amostragens em regiões de maior densidade. Assim, um dos objetivos dessa técnica é maximizar o número de regiões da amostra cobertas na estimativa.

\footnotetext{
${ }^{1}$ Denominada Control Variate em inglês.

${ }^{2}$ Denominada Stratified Sampling em inglês.
} 
Por exemplo, suponha que dividamos o intervalo $[0,1]$ em $[0, \alpha]$ e $[\alpha, 1]$. Então, se tivermos $N_{1}$ e $N_{2}$ pontos de amostragem no primeiro e segundo intervalos, respectivamente, temos o seguinte estimador

$$
\hat{I}_{f}=\frac{\alpha}{N_{1}} \sum_{i=1}^{N_{1}} f\left(x_{1 i}\right)+\frac{1-\alpha}{N_{2}} \sum_{i=1}^{N_{2}} f\left(x_{2 i}\right),
$$

onde $x_{1 i} \in[0, \alpha], i=1, \ldots, N_{1}$ e $x_{2 i} \in[\alpha, 1], i=1, \ldots, N_{2}$. Assim, sua variância é

$$
\frac{\alpha}{N_{1}} \int_{0}^{\alpha} f(x)^{2} d x-\frac{\alpha}{N_{1}}\left(\int_{0}^{\alpha} f(x) d x\right)^{2}+\frac{1-\alpha}{N_{2}} \int_{0}^{\alpha} f(x)^{2} d x-\frac{1-\alpha}{N_{2}}\left(\int_{0}^{\alpha} f(x) d x\right)^{2} .
$$

A efetividade da técnica depende da escolha do parâmetro $\alpha$. Uma boa escolha para o mesmo é aquela que iguala a variância sobre $[0, \alpha]$ com aquele sobre $[\alpha, 1]$. Assim, a ideia central nessa técnica é evitar a acumulação de pontos em uma região e, assim, reduzir a variância do estimador.

\subsection{Definições de Cálculo Estocástico}

\subsubsection{Preliminares}

Um espaço amostral ou espaço de eventos $\Omega$ é um conjunto com os possíveis resultados de experimentos ou estados de um sistema. Nesse conjunto, consideramos uma família $\mathcal{F}$ de subconjuntos de $\Omega$ e uma medida de probabilidade $\mathbb{P}: \mathcal{F} \rightarrow[0,1]$, conforme as definições a seguir.

Definição 2. A família de subconjuntos $\mathcal{F} \subset 2^{\Omega}$ é uma $\sigma$-álgebra se

i) $\emptyset \in \mathcal{F}$

ii) $\mathcal{F}$ é fechada por complementos, i.e., $F \in \mathcal{F} \Longrightarrow \bar{F} \in \mathcal{F}$.

iii) $\mathcal{F}$ é fechada por uniões enumeráveis, i.e, $\left\{F_{i}\right\}_{i=1}^{\infty} \subset \mathcal{F} \Longrightarrow \bigcup_{i=1}^{\infty} F_{i} \in \mathcal{F}$.

Definição 3. A medida de probabilidade $\mathbb{P}$ é uma função $\mathbb{P}: \mathcal{F} \rightarrow[0,1]$ tal que

i) $P(\emptyset)=0, P(\Omega)=1$

ii) Se $A_{1}, A 2, \ldots \in \mathcal{F}$ e $\left\{A_{i}\right\}_{i=1}^{\infty}$ é disjunto, então

$$
\mathbb{P}\left(\bigcup_{i=1}^{\infty} A_{i}\right)=\sum_{i=1}^{\infty} \mathbb{P}\left(A_{i}\right) .
$$

Definição 4. Uma variável aleatória $X$ é uma função $X: \Omega \rightarrow \mathbb{R}^{n}$.

Definição 5. Seja $X$ uma variável aleatória com espaço amostral $\Omega$ e medida de probabilidade $\mathbb{P}$. Então, o espaço de probabilidade gerado por $X$ é definido por

$$
\{\Omega, \mathcal{F}, \mathbb{P}\}
$$

Definição 6. Seja $\left\{X_{k}\right\}_{k=1}^{\infty}$ uma sequência de variáveis aleatórias discretas e identicamente distribuídas. Para cada inteiro positivo $n$, denotamos $S_{n}$ como a soma $X_{1}+X_{2}+\ldots+X_{n}$. A sequência $\left\{S_{n}\right\}_{n=1}^{\infty}$ é chamada de Passeio Aleatório.

Propriedade 1. Incrementos em um Passeio Aleatório são independentes e identicamente distribuídos. 


\subsubsection{Processos Estocásticos}

Suponha que a cotação do Dólar para cada instante fixo $t \in[a, b]$ seja aleatória. É razoável observarmos a evolução do preço da moeda em todo o intervalo $[a, b]$ para estimar um preço futuro. Além disso, seria interessante analisar o comportamento da trajetória aleatória para diferentes valores de partida da cotação do Dólar. Um modelo matemático para descrever tal fenômeno é chamado de processo estocástico.

Definição 7. Um Processo Estocástico X é uma coleção de variáveis aleatórias

$$
\left(X_{t}, t \in T\right)=\left(X_{t}(\omega), t \in T, \omega \in \Omega\right),
$$

definido em um espaço amostral $\Omega$.

O parâmetro $T$ é usualmente uma semi-reta $[0, \infty)$, mas também pode se referir a um intervalo $[a, b]$, o conjunto dos números naturais ou até mesmo sub-conjuntos de $\mathbb{R}^{n}$ para $n \geq 1$. O índice $t$ da variável aleatória $X_{t}$ é frequentemente referida como tempo. Podemos notar que, para um valor fixo de $t, X$ é uma variável aleatória:

$$
X_{t}=X_{t}(\omega), \omega \in \Omega .
$$

Para uma amostra aleatória $\omega \in \Omega, X$ é uma função do tempo:

$$
X_{t}=X_{t}(\omega), t \in T \text {. }
$$

Essa última função é chamada de realização, trajetória ou caminho do processo $X$.

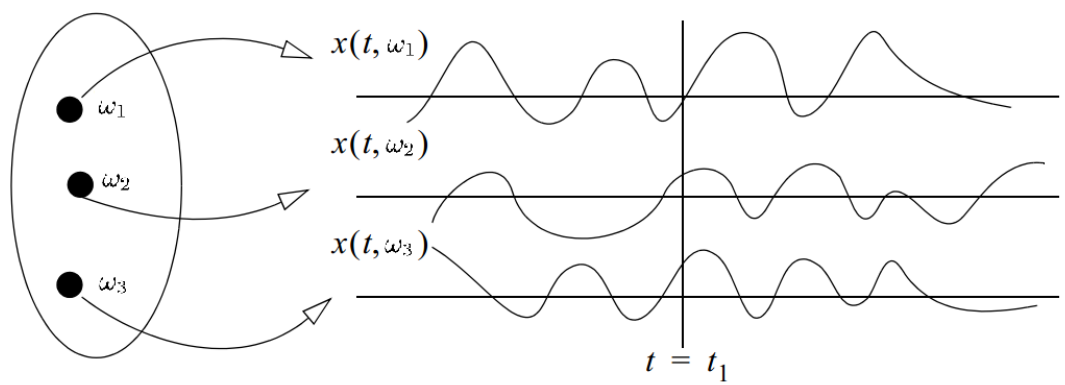

Figura 6.1: Representação de um processo estocástico. Note que para $t=t_{1}$ temos que $X\left(t_{1}\right)$ é uma variável aleatória e que para cada evento $\omega_{i}$ é gerada uma trajetória correspondente.

A esperança de um vetor de variáveis aleatórias $X=\left(X_{1}, \ldots, X_{n}\right)$ é definido como $\mu_{X}=$ $\left(\mathbb{E}\left[X_{1}\right], \ldots, \mathbb{E}\left[X_{n}\right]\right)$ e sua matriz de covariância como $\sum_{X}=\left(\operatorname{cov}\left(X_{i}, X_{j}\right), i, j=1, \ldots, n\right)$. Um processo estocástico $X=\left(X_{t}, t \in T\right)$ pode ser considerado uma coleção de vetores aleatórios $\left(X_{t}, \ldots, X_{t_{n}}\right)$ para $t_{1}, \ldots, t_{n} \in T$ e $n \geq 1$. Assim, para cada vetor de variáveis aleatórias, podemos determinar suas respectivas esperanças e matrizes de covariância. Alternativamente, podemos considerar essas grandezas como funções de $t \in T$ como na definição a seguir.

Definição 8. Seja $X=\left(X_{t}, t \in T\right)$ um processo estocástico.

A função de esperança de $X$ é dada por

$$
\mu_{X}(t)=\mathbb{E}\left[X_{t}\right], \quad t \in T .
$$

A função de covariância de $X$ é dado por

$$
c_{X}(t, s)=\operatorname{cov}\left(X_{t}, X_{s}\right)=\mathbb{E}\left[\left(X_{t}-\mu_{X}(t)\right)\left(X_{s}-\mu_{X}(s)\right)\right], \quad t, s \in T .
$$

A função de variância de $X$ é dado por

$$
\sigma_{X}^{2}(t)=c_{X}(t, t)=\operatorname{var}\left(X_{t}\right), \quad t \in T .
$$


Como para um vetor de variáveis aleatórias, a função de esperança $\mu_{X}(t)$ é uma quantidade determinística que representa a média $X$. A função de covariância $c_{X}(t, s)$ é uma medida de dependência no processo $X$. Por sua vez, a função de variância $\sigma_{X}^{2}(t)$ pode ser considerada uma medida de dispersão dos caminhos aleatórios em relação a $\mu_{X}(t)$.

\subsubsection{Movimento Browniano}

O Movimento Browniano tem um papel fundamental em teoria de probabilidade, teoria de processos estocásticos e ciências em geral. Em finanças, trata-se do processo estocástico mais comumente utilizado para simulação de preços de ativos e será base para modelagem dos estudos de casos em tempo contínuo desse trabalho. Para defini-lo, é importante enunciar o conceito de incrementos estacionários e independentes para processos estocásticos em geral.

Definição 9. Seja $X=\left(X_{t}, t \in T\right)$ um processo estocástico e $T \subset \mathbb{R}$ um intervalo. $X$ é dito ter incrementos estacionários se

$$
X_{t}-X_{s} \stackrel{d}{=} X_{t+h}-X_{s+h}, \text { para todo } t, s \in T \text { e } t+h, s+h \in T .
$$

$X$ tem incrementos independentes se para cada escolha de $t_{i} \in T$ com $t_{1}<\ldots<t_{n}$ e $n \geq 1$,

$$
X_{t_{2}}-X_{t_{1}}, \ldots, X_{t_{n}}-X_{t_{n-1}}
$$

são variáveis aleatórias independentes.

Definição 10. Um processo estocástico $B=\left(B_{t}, t \in[0, \infty)\right)$ é chamado Processo de Wiener ou Movimento Browniano Padrão se:

i) O processo tem seu início em zero: $B_{0}=0$

ii) Possui incrementos estacionários e independentes.

iii) Para todo $t>0, B_{t}$ tem uma distribuição normal $\mathcal{N}(0, t)$.

iv) O processo tem caminhos contínuos.

Segue da definição que um Movimento Browniano tem uma função de esperança

$$
\mu_{B}(t)=\mathbb{E}\left[B_{t}\right]=0, \quad t \geq 0,
$$

e como os incrementos $B_{s}-B_{0}=B_{s}$ e $B_{t}-B_{s}$ são independentes para $t>s$, sua função de covariância é

$$
\begin{aligned}
c_{B}(t, s) & =\mathbb{E}\left[\left[\left(B_{t}-B_{s}\right)+B_{s}\right] B_{s}\right]=\mathbb{E}\left[\left(B_{t}-B_{s}\right) B_{s}\right]+\mathbb{E}\left[B_{s}^{2}\right] \\
& =E\left(B_{t}-B_{s}\right) \mathbb{E}\left[B_{s}\right]+s=0+s=s, \quad 0 \leq s<t .
\end{aligned}
$$

Definição 11. Considere o processo

$$
X_{t}=\mu t+\sigma B_{t}, \quad t \geq 0,
$$

com constantes $\sigma>0$ e $\mu \in \mathbb{R}$. X é chamado de Movimento Browniano com drift (linear) e possui as seguintes funçôes de esperança e covariância, respectivamente

$$
\mu_{X}(t)=\mu t \text { e } c_{X}(t, s)=\sigma^{2} s, \quad s, t \geq 0 \text { com } s<t .
$$

O movimento browniano, como um processo Gaussiano, pode assumir valores negativos, o que pode ser uma propriedade não desejável para um preço de ativo financeiro. No célebre trabalho de (Black e Scholes, 1973), os autores sugeriram outro processo estocástico para o modelo de preços. Esse modelo é o Movimento Browniano Geométrico. 
Definição 12. Considere o processo

$$
X_{t}=e^{\mu t+\sigma B_{t}}, \quad t \geq 0,
$$

com constantes $\sigma>0$ e $\mu \in \mathbb{R} . X$ é chamado de Movimento Browniano Geométrico.

Assim, o Movimento Browniano Geométrico é a exponencial do movimento Browniano com drift.

Proposição 3. O Movimento Browniano Geométrico possui as seguintes funções de esperança e covariância, respectivamente

$$
\begin{aligned}
\mu_{X}(t) & =e^{\left(\mu+0.5 \sigma^{2}\right) t} \quad e \\
c_{X}(t, s) & =e^{\left(\mu+0.5 \sigma^{2}\right)(t+s)}\left(e^{\sigma^{2} s-1}\right), \quad s, t \geq 0 \text { com } s<t
\end{aligned}
$$

Demonstração. Temos que,

$$
B_{t}=B_{t}-B_{0} \sim N(0, \Delta t) \sim \sqrt{\Delta t} N(0,1) .
$$

Seja $Z$ uma variável aleatória tal que $Z \sim N(0,1)$, então

$$
\mu_{X}(t)=e^{\mu t} E\left[e^{\sigma B_{t}}\right]=e^{\mu t} E\left[e^{\sigma t^{1 / 2} Z}\right] .
$$

Sabemos que,

$$
E\left[e^{\lambda Z}\right]=e^{\lambda^{2} / 2}, \quad \lambda \in \mathbb{R}
$$

Então,

$$
E\left[e^{\sigma t^{1 / 2} B_{1}}\right]=e^{0.5 \sigma^{2} t}
$$

Portanto,

$$
\mu_{X}(t)=e^{\mu t} e^{0.5 \sigma^{2} t}=e^{\left(\mu+0.5 \sigma^{2}\right) t}
$$

Para $s \leq t, B_{t}-B_{s}$ e $B_{s}$ são independentes e $B_{t}-B_{s} \stackrel{d}{=} B_{t-s}$. Então,

$$
\begin{aligned}
c_{X}(t, s) & =E\left[X_{t} X_{s}\right]-E\left[X_{t}\right] E\left[X_{s}\right] \\
& =e^{\mu(t+s)} E\left[e^{\sigma\left(B_{t}+B_{s}\right)}\right]-e^{\left(\mu+0.5 \sigma^{2}\right)(t+s)} \\
& =e^{\mu(t+s)} E\left[e^{\sigma\left[\left(B_{t}-B_{s}\right)+2 B_{s}\right]}\right]-e^{\left(\mu+0.5 \sigma^{2}\right)(t+s)} \\
& =e^{\mu(t+s)} E\left[e^{\sigma\left(B_{t}-B_{s}\right)}\right] E\left[e^{2 \sigma B_{s}}\right]-e^{\left(\mu+0.5 \sigma^{2}\right)(t+s)} \\
& =e^{\left(\mu+0.5 \sigma^{2}\right)(t+s)}\left(e^{\sigma^{2} s-1}\right)
\end{aligned}
$$

\subsubsection{Simulação do Movimento Browniano}

A visualização de processos estocásticos serve como uma boa ferramenta para entendimento dos mesmos. Em muitos casos, não é possível determinar a distribuição do processo estocástico e suas propriedades. Assim, simulações e técnicas numéricas oferecem uma alternativa para o cálculo dessas distribuições. Nessa seção apresentamos uma forma de simulação do movimento Browniano. 


\section{Algoritmo Geral}

Dado um incremento fixo $\Delta t>0$, é possível simular a trajetória do processo de Wiener no intervalo $[0, T]$. De fato, para $W_{\Delta t}$ é verdade que

$$
W(\Delta t)=W(\Delta t)-W(0) \sim N(0, \Delta t) \sim \sqrt{\Delta t} N(0,1),
$$

e o mesmo também é verdade para um incremento em tempo arbitrário $W(t+\Delta t)-W(t)$ :

$$
W(t+\Delta t)-W(t) \sim N(0, \Delta t) \sim \sqrt{\Delta t} N(0,1) .
$$

Assim, podemos simular a trajetória do processo estocástico conforme Algoritmo 2.

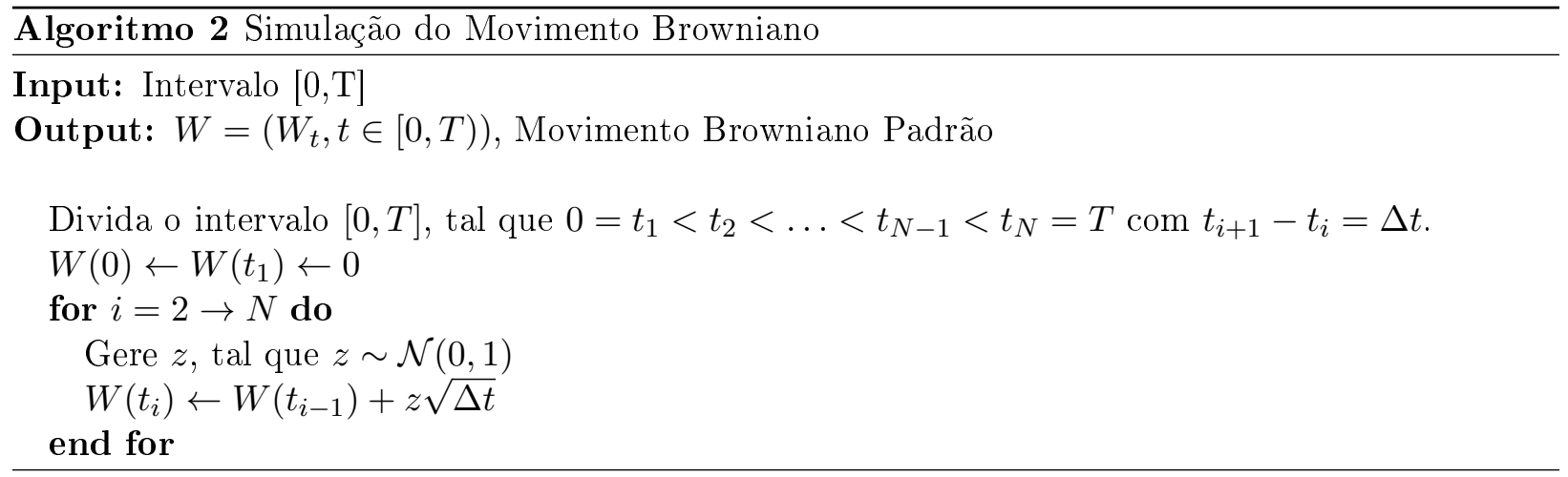

\section{Representação por Séries}

Como sabemos, uma função periódica contínua em $\mathbb{R}$ pode ser representada como uma série de Fourier, isto é, pode ser escrita como uma série infinita de funções trigonométricas. Como caminhos de um movimento Browniano são funções contínuas, é natural pensar em sua expansão em séries de Fourier. Entretanto, esses caminhos são funções aleatórias: para cada $\omega$ obtemos uma função diferente. Isso significa que os coeficientes das séries de Fourier devem ser variáveis aleatórias. A seguinte representação de um movimento Browniano no intervalo $[0,2 \pi]$ é chamado de Representação de Paley-Wiener:

$$
B_{t}(\omega)=Z_{0}(\omega) \frac{t}{(2 \pi)^{1 / 2}}+\frac{2}{\pi^{1 / 2}} \sum_{n=1}^{\infty} Z_{n}(\omega) \frac{\sin (n t / 2)}{n}, \quad t \in[0,2 \pi],
$$

onde $\left(Z_{n}, n \geq 0\right)$ corresponde a uma sequência de variáveis aleatórias gaussianas independentes.

Para uma aplicação dessa fórmula, é necessário decidir sobre o número $M$ de funções seno e o número $N$ de pontos de discretização nos quais essas funções serão realizadas:

$$
Z_{0}(\omega) \frac{t}{(2 \pi)^{1 / 2}}+\frac{2}{\pi^{1 / 2}} \sum_{n=1}^{M} Z_{n}(\omega) \frac{\sin \left(n t_{j} / 2\right)}{n}, \quad t_{j}=\frac{2 \pi j}{N}, \quad j=0,1, \ldots, N .
$$

Na verdade, a Representação de Paley-Wiener é uma de infinitas séries possíveis de representação de um movimento Browniano. Ela é um caso especial da chamada Representação de Lévy-Ciesielski (Hida, 1980). Ciesielski mostrou que um movimento Browniano em $[0,1]$ pode ser representado na forma

$$
B_{t}(\omega)=\sum_{n=1}^{\infty} Z_{n}(\omega) \int_{0}^{t} \phi_{n}(x) d x
$$

onde $Z_{n}$ corresponde a uma sequência de variáveis aleatórias gaussianas independentes no intervalo 
$[0,1]$ e $\phi_{n}(x)$ deve possuir algumas propriedades especiais, como a formação de um sistema completo de funções ortonormais (Kaplan, 1991).

\subsection{Equações Diferenciais Estocásticas}

\subsubsection{Integral de Itô}

Definição 13. A integral de Itô é definida como

$$
\int_{0}^{t} B(\tau) d W(\tau)=\lim _{n \rightarrow \infty} \sum_{k=0}^{n-1} B\left(t_{k}\right)\left[W\left(t_{k+1}\right)-W\left(t_{k}\right)\right], \text { onde } t_{k}=k \frac{t}{n}
$$

No caso particular onde $B(t)$ é uma função determinística, essa integral é chamada de Integral de Wiener.

Considere a seguinte equação

$$
X(t)=X(0)+\int_{0}^{t} a(X(\tau), \tau) d \tau+\int_{0}^{t} b(X(\tau), \tau) d W(\tau) .
$$

Em um intervalo infinitesimal, podemos re-escrever essa equação em sua forma diferencial:

$$
d X(t)=a(X(t), t) d t+b(X(t), t) d W(t),
$$

onde $W(t)$ representa um processo de Wiener e $a(X(t), t)$ e $b(X(t), t)$ são, respectivamente, as variações instantâneas da média e desvio padrão.

Genericamente, podemos escrever

$$
X(t)=X(0)+\int_{0}^{t} A(\tau) d \tau+\int_{0}^{t} B(\tau) d W(\tau),
$$

onde $A(\tau)$ e $B(\tau)$ são funções de $X(t)$ para $0 \leq \tau \leq t$.

Estamos interessados no caso em que

$$
\int_{0}^{t} \mathbb{E}[|A(\tau)|] d \tau+\int_{0}^{t} \mathbb{E}\left[|B(\tau)|^{2}\right] d \tau<\infty .
$$

Processos que são soluções dessa equação são chamados de Processos de Itô.

\section{Propriedades de um Processo de Itô}

Propriedade 2. Se $X$ é um processo de Itô, então

$$
\mathbb{E}\left[\int_{0}^{t} X(\tau) d W(\tau)\right]=0
$$

$e$

$$
\operatorname{Var}\left(\int_{0}^{t} X(\tau) d W(\tau)\right)=\int_{0}^{t} E\left[X^{2}(\tau) d \tau\right]
$$

Propriedade 3. Linearidade

Se $X$ e $Y$ são dois processos de Itô e a e b duas constantes, então

$$
\int_{0}^{t}(a X(\tau)+b Y(\tau)) d W(\tau)=a \int_{0}^{t} X(\tau) d W(\tau)+b \int_{0}^{t} Y(\tau) d W(\tau) .
$$


Propriedade 4. Segue da propriedade de linearidade anterior que

$$
\int_{0}^{t} a W(\tau)=a \int_{0}^{t} d W(\tau)=a W(t)
$$

\subsubsection{Lema de Itô}

Considere $X$ um processo uni-dimensional definido como:

$$
d X(t)=a(X(t), t) d t+b(X(t), t) d W(t) .
$$

Seja $Y(t)=g(t, X(t))$, onde $g$ é duplamente diferenciável e contínua. Então, o Lema de Itô diz que

$$
\begin{aligned}
d Y & =\left(\frac{\partial g}{\partial t}+a(X(t), t) \frac{\partial g}{\partial x}+\frac{1}{2} b^{2}(X(t), t) \frac{\partial^{2} g}{\partial x^{2}}\right) d t \\
& +\left(b(X(t), t) \frac{\partial g}{\partial x}\right) d W .
\end{aligned}
$$

Similarmente ao caso uni-dimensional, a mesma regra aplica-se para o caso multi-dimensional. Seja $\bar{X} \in \mathbb{R}^{n}$ um vetor de variáveis aleatórias com processo definido como

$$
d \bar{X}(t)=A(\bar{X}(t), t) d t+B(\bar{X}(t), t) d \bar{W}(t) .
$$

onde $A(\bar{X}(t), t) \in \mathbb{R}^{n}, \bar{W} \in \mathbb{R}^{n}$ e $B(\bar{X}(t), t) \in \mathbb{R}^{n \times m}$.

Fazendo

$$
\bar{Y}(t)=\bar{g}(t, \bar{X}(t))=\left(Y_{1}\left(t, \ldots, Y_{d}(t)\right)\right)^{T}
$$

$\operatorname{com} \bar{g}: \mathbb{R} \times \mathbb{R}^{n} \rightarrow \mathbb{R}^{d}$, então o Lema de Itô generalizado é

$$
d Y_{k}(t)=\frac{\partial g_{k}}{\partial t} d t+\sum_{i} \frac{\partial g_{k}}{\partial x_{i}} d X_{i}(t)+\frac{1}{2} \sum_{i} \frac{\partial^{2} g_{k}}{\partial x_{i} \partial x_{j}} d X_{i}(t) d X_{j}(t)
$$

A seguir, demonstramos a proposição 4, como exemplo de aplicação do Lema de Itô enunciado.

\section{Proposição 4.}

$$
\int_{0}^{t} W(\tau) d W(\tau)=\frac{1}{2} W^{2}(t)-\frac{1}{2} t .
$$

Demonstração. Seja $X(t)=W(t)$, escolha $g$ tal que

$$
\begin{aligned}
Y(t) & =g(t, X(t)) \\
& =\frac{1}{2} W^{2}(t) .
\end{aligned}
$$

Aplicando o Lema de Itô, temos

$$
d Y(t)=W(t) d W(t)+\frac{1}{2} d t
$$

Assim,

$$
\begin{aligned}
\int_{0}^{t} W(\tau) d W(\tau) & =Y(t)-Y(0)-\frac{1}{2} t \\
& =\frac{1}{2} W^{2}(t)-\frac{1}{2} t .
\end{aligned}
$$




\section{Processo de Ornstein-Uhlenbeck}

Em finanças, o processo de Ornstein-Uhlenbeck é um processo de reversão à média ${ }^{3}$ muito comum para descrever a dinâmica de taxas de juros e volatilidades estocásticas de retornos de ativos. Sua definição é dada pela equação a seguir:

$$
d X(t)=\theta(\mu-X(t)) d t+\sigma d W(t),
$$

onde $W(t)$ é um processo padrão de Wiener, $\sigma$ é o parâmetro de volatilidade do processo e representa o nível de intensidade das perturbações estocásticas, $\mu$ é o valor ao qual o processo possui reversão e $\theta$ representa a velocidade dessa reversão.

A seguir, descrevemos a solução do processo de Ornstein-Uhlenbeck, cujo desenvolvimento pode ser encontrado em (Alves, 2008).

Proposição 5. A solução do processo de Ornstein-Uhlenbeck definido pela equação 6.14 é dado por

$$
X(t)=\mu+(X(0)-\mu) e^{-\theta t}+\sigma \int_{0}^{t} e^{-\theta(t-s)} d W(s)
$$

Demonstração. Temos que,

$$
\begin{aligned}
d X(t) & =\theta(\mu-X(t)) d t+\sigma d W(t), \\
d X(t)+\theta X(t) d t & =\theta \mu d t+\sigma d W(t) \\
d\left(e^{\theta t} X(t)\right) & =\theta \mu e^{\theta t}+\sigma e^{\theta t} d W(t) .
\end{aligned}
$$

Integrando em $t$, temos

$$
\int_{0}^{t} d\left(e^{\theta s} X(s)\right) d s=\int_{0}^{t} \theta \mu e^{\theta s} d s+\sigma \int_{0}^{t} e^{\theta s} d W(s) .
$$

Assim,

$$
X(t) e^{\theta t}-X(0)=\mu e^{\theta t}-\mu+\sigma \int_{0}^{t} e^{\theta s} d W(s)
$$

Portanto,

$$
X(t)=\mu+(X(0)-\mu) e^{-\theta t}+\sigma \int_{0}^{t} e^{-\theta(t-s)} d W(s)
$$

\subsection{Soluções Numéricas de Equações Diferenciais Estocásticas}

Antes de introduzir soluções numéricas de equações diferenciais estocásticas, apresentaremos discretizações numéricas de equação diferenciais ordinárias, como um ponto de partida. Isso facilitará o entendimento das discretizações de Euler-Maruyama e Milstein que serão cobertas nas seções posteriores.

\footnotetext{
${ }^{3}$ Reversão à média é um conceito matemático utilizado em investimentos financeiros para designar que um fator de risco tende a um valor médio.
} 


\subsubsection{Equações Diferenciais Ordinárias}

Considere a seguinte equação diferencial ordinária

$$
d x=a(x, t) d t, \quad x\left(t_{0}\right)=x_{0} .
$$

Definimos,

$$
t_{n+1}=t_{n}+\Delta t, \quad x\left(t_{n+1}\right)=x_{n}+\delta x=x_{n+1}
$$

e $y_{n+1}=y\left(t_{n+1}\right)$ como a aproximação linear de $x_{n+1}$, ou seja,

$$
y\left(t_{n}+\Delta t\right) \approx y\left(t_{n}\right)+\frac{d y}{d t} \Delta t .
$$

\section{Método de Euler}

A aproximação de Euler considera a seguinte discretização

$$
y\left(t_{n}+\Delta t\right) \approx y\left(t_{n}\right)+\frac{d y}{d t} \Delta t
$$

que corresponde a uma aproximação de primeira ordem da série de Taylor.

\section{Método de Runge-Kutta}

O método de Runge-Kutta é baseado na seguinte aproximação

$$
y_{n+1}=y_{n}+\frac{1}{6}\left(k_{n}^{1}+2 k_{n}^{2}+2 k_{n}^{3}+k_{n}^{4}\right) \Delta t
$$

onde

$$
\begin{aligned}
k_{n}^{1} & =a\left(y_{n}, t_{n}\right), \\
k_{n}^{2} & =a\left(y_{n}+\frac{1}{2} k_{n}^{1} \Delta t, t_{n}+\frac{1}{2} \Delta t\right), \\
k_{n}^{3} & =a\left(y_{n}+\frac{1}{2} k_{n}^{2} \Delta t, t_{n}+\frac{1}{2} \Delta t\right), \\
k_{n}^{4} & =a\left(y_{n}+k_{n}^{3} \Delta t, t_{n+1}\right) .
\end{aligned}
$$

Esse é conhecido como algoritmo de quarta-ordem de Runge-Kutta. O mesmo possui erro de $O\left(\Delta t^{4}\right)$.

\section{Método Implícito}

Nesse método, consideramos a média

$$
\frac{a\left(y_{n}, t_{n}\right)+a\left(y_{n+1}, t_{n+1}\right)}{2},
$$

que leva a aproximação numérica

$$
y_{n+1}=y_{n}+\frac{a\left(y_{n}, t_{n}\right)+a\left(y_{n+1}, t_{n+1}\right)}{2} \Delta t .
$$

Enquanto que Euler e Runge-Kutta são métodos de aproximação explícita, esse método é implícito pois a solução é obtida de modo iterativo. 


\subsubsection{Equações Diferenciais Estocásticas}

Considere a seguinte equação diferencial estocástica

$$
\left\{\begin{array}{l}
d S(t)=a(S(t), t) d t+b(S(t), t) W(t), t \in(0, T] \\
S(0)=S_{0}
\end{array}\right.
$$

onde $b: \mathbb{R}^{d} \times[0, T] \rightarrow \mathbb{R}^{d}$ é conhecido e $W(\cdot)$ representa um processo de Wiener. Um método padrão para mostrar a existência de solução para o sistema de equações de 6.15 é fornecido em (Narayana et al., 2012) e apresentado a seguir.

Seja

$$
S^{i+1}(t)=S_{0}+\int_{0}^{t} a\left(S^{i}(u)\right) d u+\int_{0}^{t} b\left(S^{i}(u)\right) d W(u)
$$

para $i \geq 1$ e $S^{0}=S_{0}$ para todo $i$. Então, $S^{i} \rightarrow S$ quando $i \rightarrow \infty$, onde $S$ é a solução da equação 6.15 .

Assim, a equação 6.16 fornece uma forma de obtenção de uma solução numérica das equações em 6.15. Há dois métodos principais na literatura de como aproximar as integrais enunciadas: a Discretização de Euler e a Discretização de Milstein.

\section{Discretização de Euler-Maruyama}

Para $N \in \mathbb{N}$, seja $h=\frac{T}{N}$. Denotemos $S_{i}, W_{i}, a_{i}$ para o $i$-ésimo componente de $S, W, a$ e $b_{i j}$ para a $i j$-ésima entrada de $b$.

Seja

$$
\begin{aligned}
S_{i}((k+1) h) & =S_{i}(k h)+\int_{k h}^{(k+1) h} a_{i}(S(u)) d u \\
& +\sum_{j}^{m} \int_{k h}^{(k+1) h} b_{i j}(S(u)) d W_{j}(u)
\end{aligned}
$$

Na discretização de Euler, a integral é aproximada como

$$
\int_{k h}^{(k+1) h} a_{i}(S(u)) d u \approx a_{i}(S(k h)) h
$$

e a Integral de Itô como

$$
\int_{k h}^{(k+1) h} b_{i j}(S(u)) d W_{j}(u) \approx b_{i j}(S(k h))\left[W_{j}((k+1) h)-W_{j}(k h)\right]
$$

\section{Discretização de Milstein}

A diferença da Discretização de Milstein para Discretização de Euler está na forma como a Integral de Itô é aproximada. Na Discretização de Euler, não são considerados os termos de maior ordem na série de expansão de Taylor da Integral de Itô, enquanto a Discretização de Milstein os consideram. Isso faz com que o esquema de Milstein seja mais preciso e tenha maior taxa de convergência.

Para $N \in \mathbb{N}$, seja $h=\frac{T}{N}$. Denotemos $S_{i}, W_{i}, a_{i}$ para o $i$-ésimo componente de $S, W, a$ e $b_{i j}$ para a $i j$-ésima entrada de $b$. 
Seja

$$
\begin{aligned}
S_{i}((k+1) h) & =S_{i}(k h)+\int_{k h}^{(k+1) h} a_{i}(S(u)) d u \\
& +\sum_{j}^{m} \int_{k h}^{(k+1) h} b_{i j}(S(u)) d W_{j}(u) .
\end{aligned}
$$

Na discretização de Milstein, a integral é aproximada como

$$
\int_{k h}^{(k+1) h} a_{i}(S(u)) d u \approx a_{i}(S(k h)) h
$$

e a Integral de Itô como

$$
\begin{gathered}
\int_{k h}^{(k+1) h} b_{i j}(S(u)) d W_{j}(u) \approx b_{i j}(S(k h))\left[W_{j}((k+1) h)-W_{j}(k h)\right] \\
+\sum_{l=1}^{d} \sum_{m=1}^{d} \frac{\partial b_{i j}}{\partial x_{l}}(S(k h)) b_{l m} \int_{k h}^{(k+1) h}\left[W_{m}(u)-W_{m}(k h)\right] d W_{j}(u)
\end{gathered}
$$

Uma dedução da aproximação de Milstein pode ser obtida em (Mikosch, 1999).

\section{Cálculo da Esperança}

Se tomarmos $a(S(t), t)=\mu S_{t}$ e $b(S(t), t)=\sigma S_{t}$ na Equação 6.15 , então chegamos ao seguinte modelo

$$
\left\{\begin{array}{l}
d S_{t}=\mu S_{t} d t+\sigma S_{t} W(t), t \in\left(t_{0}, T\right] \\
S\left(t_{0}\right)=S_{0}
\end{array}\right.
$$

Os possíveis valores assumidos por $S_{t}$ são, então, gerados ao sub-dividir o tempo $T-t_{0}$ em $M$ passos temporais discretos de tamanho $\delta t=\frac{T-t_{0}}{M}$.

Assim, pela discretização de Euler chegamos a

$$
S_{i+1}=S_{i}+\mu S_{i} \delta t+\sigma S_{i} \sqrt{\delta t} x
$$

Utilizando a discretização de Milstein temos

$$
S_{i+1}=S_{i}+\mu S_{i} \delta t+\sigma S_{i} \sqrt{\delta t} x+\frac{1}{2} \sigma^{2} S_{i}\left(\delta t x^{2}-\delta t\right)
$$

onde $x \sim \mathcal{N}(0,1)$ e $i=1, \ldots, M$.

Com isso, podemos construir o Algoritmo 3 para estimar $\theta=E\left[f\left(S_{t}\right)\right]$, ou seja, a esperança de uma função sobre a variável aleatória $S_{t}$. O algoritmo supõe um número de caminhos $n$ fixo e um intervalo de discretização $h$.

Por meio da equação 6.22 , podemos construir algoritmo análogo para discretização de Milstein. 


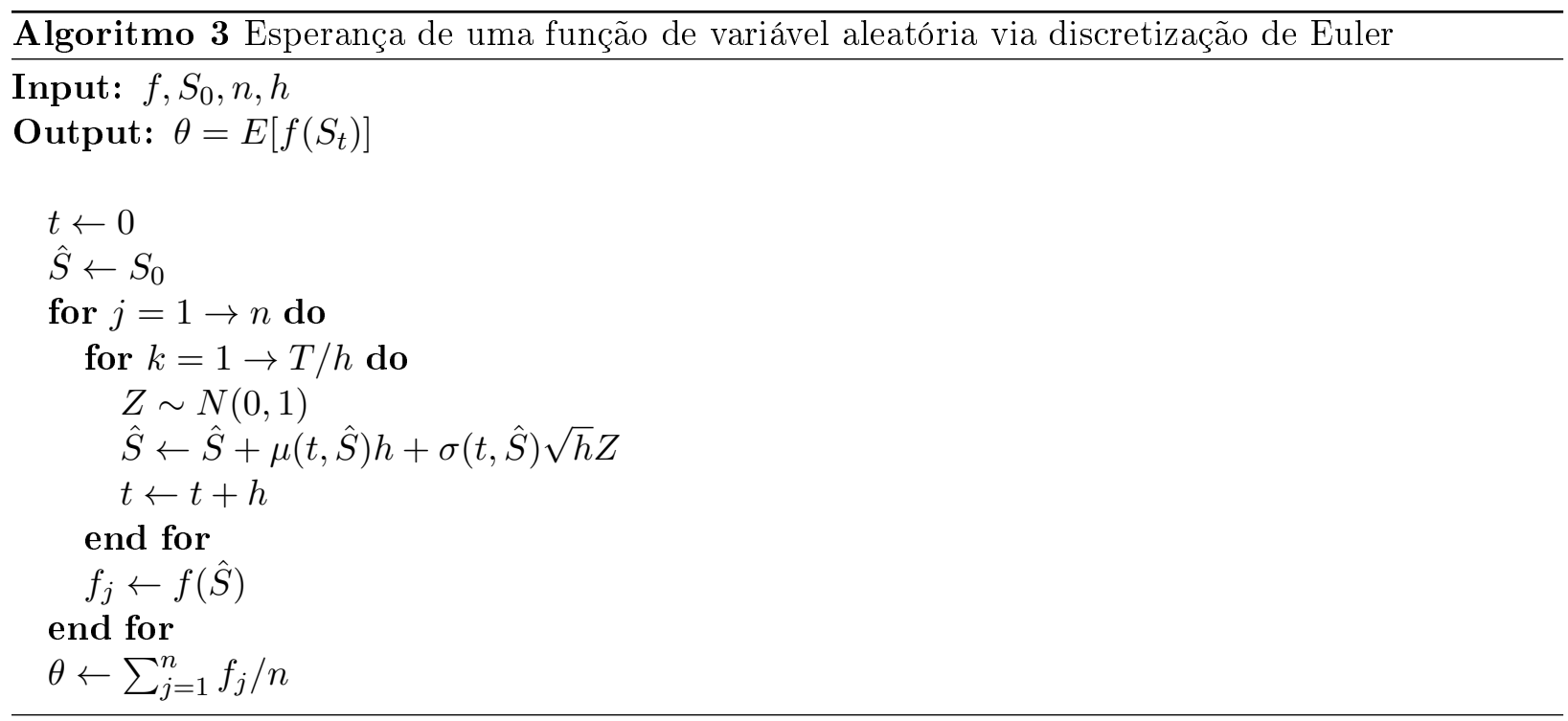




\section{Parte IV}

\section{Estudo de Casos}





\section{Capítulo 7}

\section{Método}

Este capítulo descreve o ambiente computacional e a configuração de execução da análise experimental dos estudos de casos das próximas seções. A configuração aqui enunciada foi utilizada em todos os experimentos das próximas seções a menos que seja dito o contrário.

\subsection{Ambiente Computacional}

\subsubsection{GPU}

O código em GPU foi desenvolvido em CUDA C utilizando o compilador nvcc (CUDA Toolkit v.3.1) com a opção -use_fast_math para otimização de funções matemáticas. Foi utilizado uma placa gráfica NVIDIA GeForce GT 525M de arquitetura Fermi, que possui 96 núcleos CUDA. A tabela 7.1 sumariza as principais características da mesma.

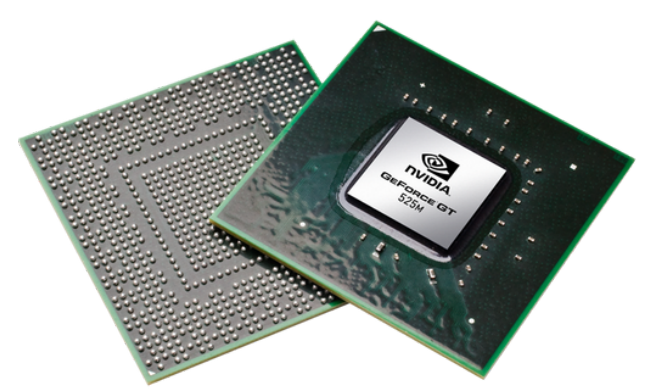

Figura 7.1: NVIDIA GeForce GT 525M

\begin{tabular}{|l|c|}
\hline Placa Gráfica & GeForce GT 525M \\
\hline Núcleos CUDA & 96 \\
\hline Compute Capability & 2.1 \\
\hline Streaming Multiprocessors & 2 \\
\hline Dimensões máximas de um bloco & $1024 \times 1024$ x 64 \\
\hline Número máximo de threads por bloco & 1024 \\
\hline Número máximo de registradores por bloco & 32768 \\
\hline Memória Global & $961 \mathrm{MB}$ \\
\hline Memória Compartilhada & $48 \mathrm{~KB}$ \\
\hline Memória Constante & $64 \mathrm{~KB}$ \\
\hline
\end{tabular}

Tabela 7.1: Características da placa gráfica NVIDIA GeForce GT 525M. 


\subsubsection{CPU}

O código em CPU foi desenvolvido em $\mathrm{C} / \mathrm{C}++$ utilizando o compilador Microsoft ( $R$ ) $\mathrm{C} / \mathrm{C}++$ Optimizing Compiler Version 16.00.30319.01 for x64 em um processador Intel Core i5-2430M. Os programas em CPU foram construídos utilizando uma única thread.

\subsection{Tempo Computacional e Medidas}

O tempo computacional foi medido com temporizadores de CPU, conforme elucidado na seção 4.1. Todos os tempos foram medidos considerando todo o tempo gasto no programa, ou seja, também foi considerado o tempo de transferência de dados via PCIe, no caso de GPU. A métrica de desempenho utilizada foi a de speedup.

O erro padrão calculado nas simulações considerou o intervalo a um nível de confiança de $95 \%$.

\subsection{Dados Disponíveis}

Os preços das ações utilizadas nos experimentos foram retirados do site da BM\&FBovespa (http://www.bmfbovespa.com.br/). Foram considerados os preços de fechamento do ativo.

\subsection{Geração de Números Aleatórios}

Em CPU, o gerador padrão utilizado foi o Mersenne Twister, com implementação retirada de (Matsumoto e Nishimura, 2009). Em GPU foi utilizada a biblioteca padrão NVIDIA CURAND. Nos experimentos que utilizaram o gerador Sobol, a implementação foi obtida em (Joe e Kuo, 2008). Para transformada gaussiana foi utilizado o método de Box-Muller.

\subsection{Configuração de Execução}

Seguindo as boas práticas enunciadas no capítulo 4, as funções de GPU foram lançadas com um número de threads por bloco como um múltiplo de 64. Empiricamente, o valor que resultou em maior ocupação, em geral, foi o de 128 threads por blocos. Esse foi o valor utilizado em todos os estudos de caso.

A figura 7.2 ilustra a configuração básica de execução dos experimentos realizados. Primeiramente, medimos o tempo do experimento em CPU. Em seguida, iniciamos a contagem de tempo de cálculos em GPU. De modo geral, a paralelização é feita na geração dos números aleatórios em GPU e também na dimensão das simulações realizadas. 
HOST

\section{DEVICE}

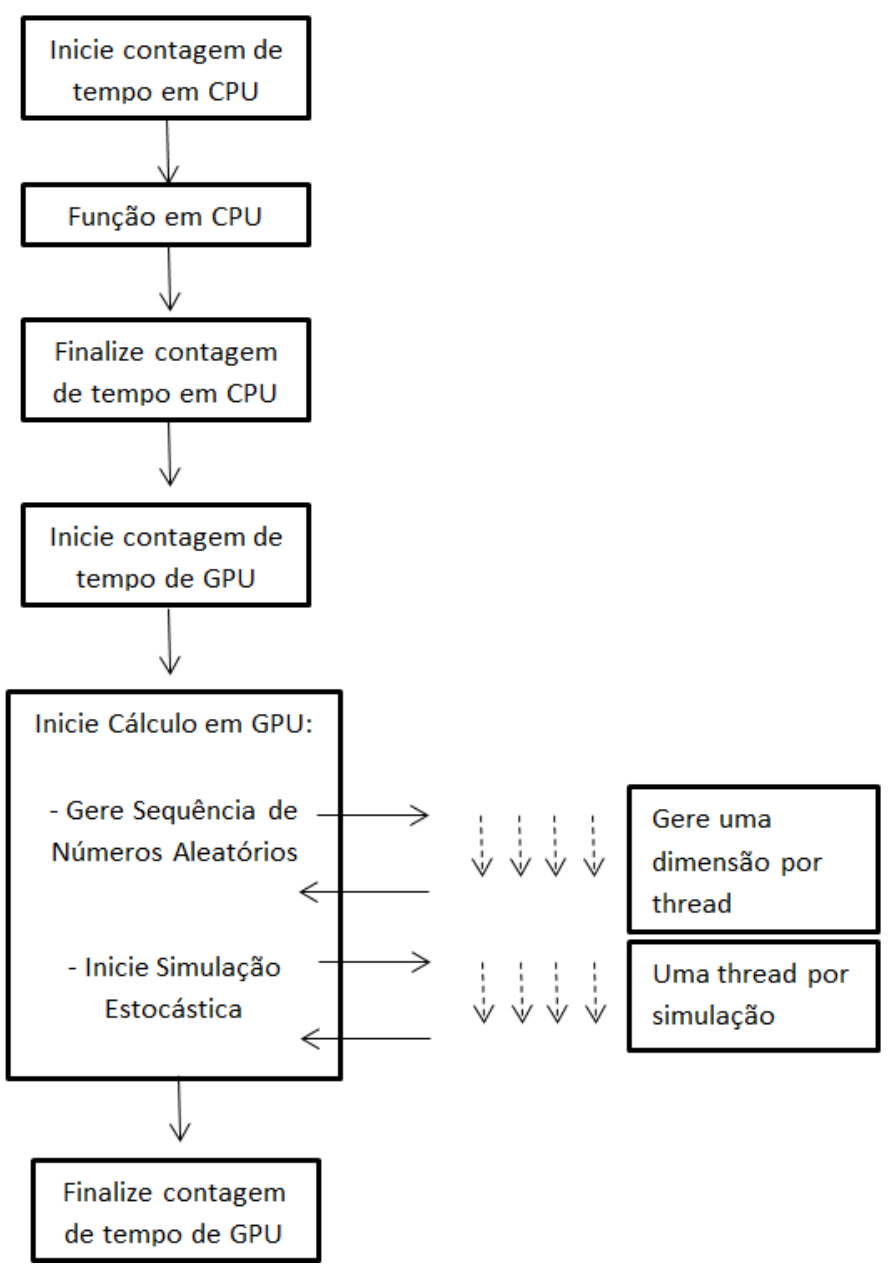

Figura 7.2: Arquitetura geral de execução de um estudo de caso. 


\section{Capítulo 8}

\section{Stops Ótimos}

A decisão de venda de um ativo é crucial para o sucesso em investimentos no mercado financeiro. A estratégia de venda pode ser determinada, por exemplo, tanto por um ganho alvo ou um limite de perda. Em negociação de ações, uma ordem de Stop Loss tenta vender o ativo quando seu preço da ação cai a um certo valor pré-determinado. Assim, a estratégia de Stop Loss é modelada para limitar as perdas a uma região segura. De modo análogo, na estratégia de Stop Gain, o negociador define um limite superior para o valor do ativo de negociação. Ao ser atingido esse nível, o investidor deixa sua posição e tem seu ganho conforme pré-determinado. Há dois tipos de Stop: fixo ou móvel. No primeiro caso, um valor alvo é pré-determinado na ordem realizada. No caso de um Stop móvel, o preço para executar a ordem pode variar com o tempo.

A utilização de uma estratégia financeira de Stop pode ser justificada por diferentes razões como:

- Redução da frequência de negociação e, consequentemente, dos custos totais de operação

- Fornece uma maneira simples de controle de perdas ou ganhos

- Em caso de negociação automatizada, permite níveis de recalibração do modelo de estratégia empregado

Funções de ganho similares aos resultantes de uma estratégia de Stop podem ser obtidas por simulação correspondente em Opções com Barreiras. Entretanto, essas Opções não são comumente negociadas em Bolsa de Valores. Assim, negociadores de varejo, geralmente, devem recorrer a técnicas de Stop como proteção alternativa. Veja (Hull, 2012) para uma introdução a Opções com Barreiras.

(Zhang, 2001) determina uma regra de venda ótima para um ativo cujo retorno segue um modelo estocástico correlacionado ao retorno de um portfólio de mercado. (Shen e Wang, 2001) consideram uma ordem de Stop Loss móvel que incrementa com o avanço do tempo. Nesse trabalho, não são considerados efeitos de portfólio em caso de configuração de Stop por todos os participantes do mercado.

(Imkeller e Rogers, 2010) realizam uma modelagem estocástica no qual o retorno da posição segue um movimento Browniano. São analisadas as seguintes situações: (i) Stops Fixos; (ii) Stop Gain Móvel; (iii) Stop Gain Móvel com Stop Loss Fixo; (iv) Stops Convergentes: onde a diferença entre os limites superior e inferior tendem a um valor fixo. Ao analisar cada um desses cenários, os autores apresentaram as seguintes conclusões: (i) a incerteza sobre a taxa de retorno do ativo é determinante na escolha dos Stops; (ii) somente há necessidade de Stop Loss caso uma taxa de retorno prevista seja negativa; (iii) ao utilizar um Stop superior fixo, não houve diferenças significativas entre Stops inferiores fixos ou de subida. Contudo, o tempo médio em negociação é significativamente menor em uma estratégia de Stop Loss de subida. Assim, a estratégia recomendada pelos autores é a de Stop superior fixo com Stop inferior de subida.

Em (Warburtona e Zhang, 2006), os autores definem um modelo computacional em tempo discreto para análise probabilística de Stops fixos. Além de analisar as estratégias de Stop Loss e Stop 
Gain, os autores estudam a definição de pontos de entrada de negociação, ou seja, o usuário inicia ou termina negociação dependendo de limites definidos para o valor do ativo.

Na seção 8.1 formalizamos o problema de Stops. Na seção 8.2 discutimos o modelo em tempo discreto proposto por (Warburtona e Zhang, 2006) e apresentamos um algoritmo paralelo em CUDA baseado nesse trabalho. Na seção 8.3 descrevemos um modelo estocástico simplificado para o problema, bem como um algoritmo em CUDA para sua resolução. Finalmente, na seção 8.5, realizamos uma análise experimental das modelagens propostas.

\subsection{Formulação do Problema}

Suponha que uma partícula se move aleatoriamente em um sub-conjunto $D \subset \mathbb{R}^{n}$. No instante $t$ sua posição é $x_{t}$. Desejamos escolher o Tempo de Parada $t$ do movimento de modo a maximizar o ganho esperado $\mathbb{E}\left[g\left(x_{t}\right)\right]$, onde $g(x)$ representa o ganho correspondente a $x \in D$ (vide figura 8.1).

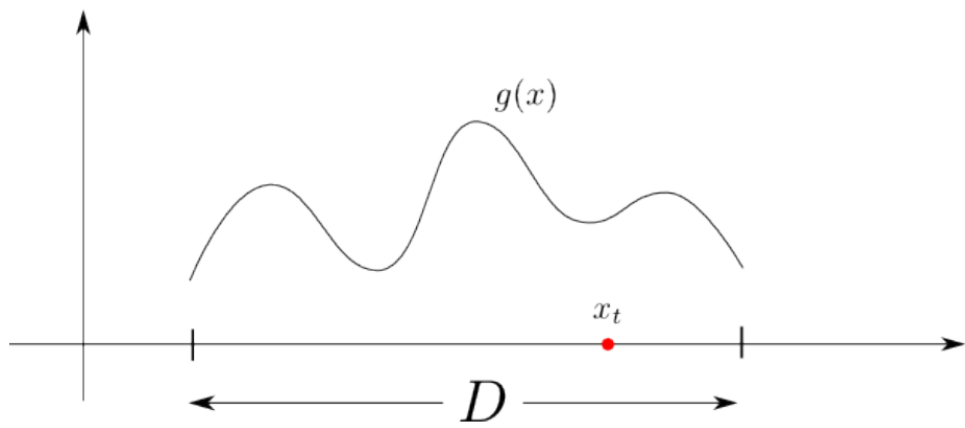

Figura 8.1: O problema de Stop

O termo Tempo de Parada tem sua origem na teoria de apostas. A variável $x_{t}$ pode representar o ganho acumulado (ou perda) de um apostador no tempo $t$. Um apostador pragmático pode desejar uma regra para decidir no tempo $t$ se é hora de sair do jogo ou não. Como jogadores, geralmente, não têm a capacidade de prever o futuro, essa regra deve se basear somente no histórico do jogo até o momento.

Para formular o problema rigorosamente, necessitamos de uma definição formal para o conceito de Tempo de Parada.

Definição 14. Tempo de Parada (Oksendal, 1992)

Seja $\left\{N_{t}\right\}$ uma familia crescente de $\sigma$-álgebras (de sub-conjuntos de $\Omega$ ). A função $\tau: \Omega \rightarrow[0, \infty]$ é chamado de tempo de parada se

$$
\{\omega ; \tau(\omega) \leq t\} \in N_{t}, \quad \forall t \geq 0 .
$$

Assim, intuitivamente, a decisão de parar no instante $t$ deve depender apenas da informação ( $\sigma$-álgebra) disponível até $t$. A figura 8.2 ilustra exemplos de Tempo de Parada.

Dessa forma, nosso problema pode ser formalizado como:

$$
\max \mathbb{E}^{x_{0}}\left[g\left(x_{\tau}\right)\right]
$$

$$
\text { sujeito a } \tau \text { tempo de parada }
$$

Com isso, $g\left(x_{\tau}\right)$ é o ganho que obteremos ao parar o processo no tempo $\tau$. Desejamos maximizar o valor esperado do ganho para as trajetórias que tem inicio em $x_{0}$. A variável do problema é $\tau$, um tempo de parada, ou seja, temos um problema de otimização no espaço abstrato dos tempos de parada. 


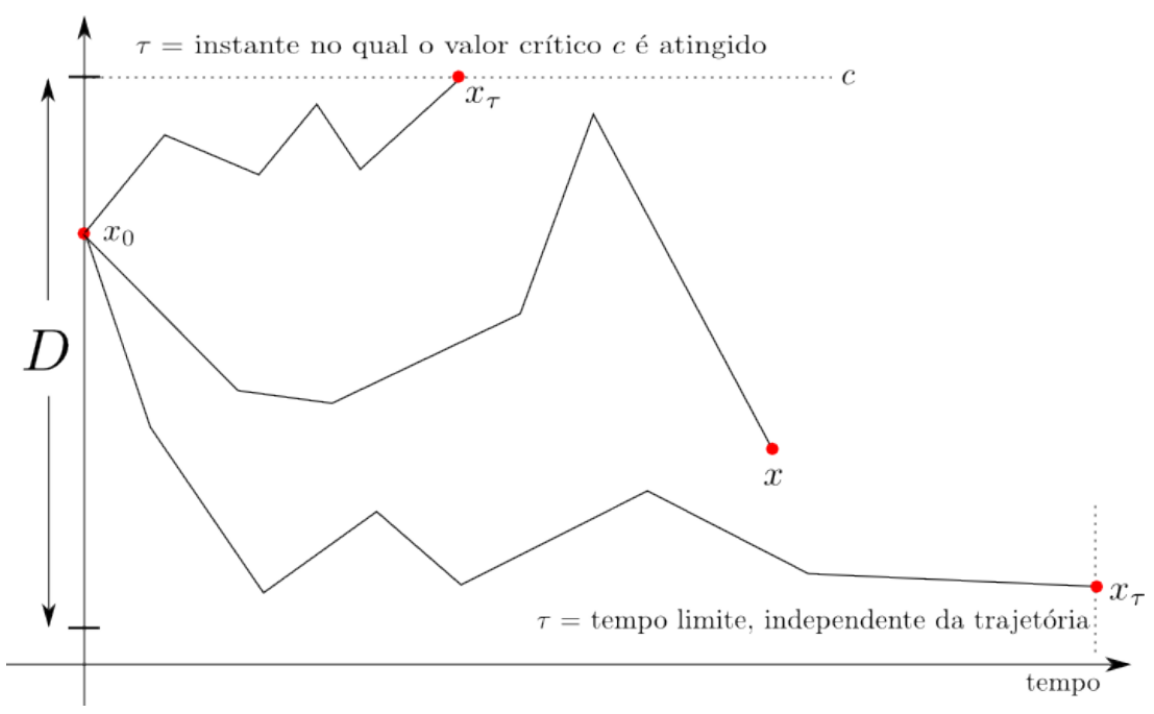

Figura 8.2: Exemplos de Tempo de Parada

\subsection{Modelagem em Tempo Discreto}

\subsubsection{O Modelo Recursivo de (Warburtona e Zhang, 2006)}

Em (Warburtona e Zhang, 2006), os autores propõem um modelo baseado em uma árvore trinomial para o passeio aleatório dos preços. Dessa forma, o valor do ativo pode subir um nível, continuar constante ou descer um nível. O processo para assim que uma barreira é atingida ou ao final do horizonte de tempo. O modelo apresenta as seguintes premissas:

(i) Preço do ativo segue um passeio aleatório

(ii) Horizonte de tempo é finito

(iii) Stops são fixos

Sejam,

- $T$ : o número total de intervalos de tempo no horizonte de tempo indexados por $t=0,1, \ldots, T$

- $\Delta$ : o tamanho de cada intervalo de tempo

- $H$ : o tamanho do horizonte de tempo, $H=T \Delta$

- L: o valor de Stop Loss (barreira inferior)

- K: o valor de Stop Gain (barreira superior)

- $\Omega$ : o espaço de probabilidade de eventos (nível de preço $x$ a cada instante $t$ )

- $P(t, x)$ : probabilidade do processo estar no estado $(t, x)$

- $S(t, x)$ : o preço do ativo se o processo está no estado $(t, x)$

- $p(t, x), q(t, x), r(t, x)$ : as probabilidades do preço subir um nível, continuar inalterado, e descer um nível, respectivamente, no estado $(t, x) \cdot p(t, x)+q(t, x)+r(t, x)=1$

Considere, $B(t, x)$ como os predecessores diretos de $(t, x)$ :

$$
B(t, x)=\{(t-1, y):(t-1, y) \in \Omega, y \in\{x-1, x, x+1\}\}
$$


Seja $I_{B(t, x)}(t-1, y)$ a função booleana definida por

$$
I_{B(t, x)}(t-1, y)= \begin{cases}1, & \text { se }(t-1, y) \in B(t, x) \\ 0, & \text { caso contrário }\end{cases}
$$

Então, a seguinte função de probabilidade é definida para computar $P(t, x),(t, x) \in \Omega$ :

$$
\begin{aligned}
P(0,0) & =1 \\
P(t, x) & =P(t-1, x-1) I_{B(t, x)}(t-1, x-1) p(t-1, x-1) \\
& +P(t-1, x) I_{B(t, x)}(t-1, x) r(t-1, x) \\
& +P(t-1, x+1) I_{B(t, x)}(t-1, x+1) q(t-1, x+1), \\
& (t, x) \in \Omega \backslash(0,0) .
\end{aligned}
$$

A figura 8.3 exemplifica movimentos possíveis para os preços do ativo.

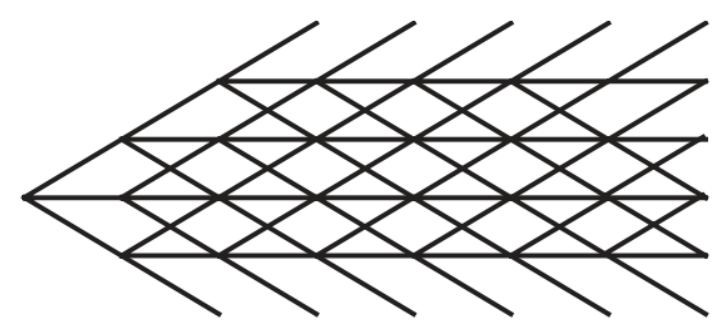

Figura 8.3: Movimentos de preço possiveis para $T=7, K=3, L=-2$

Um investimento termina assim que um nó sorvedouro é atingido. Seja, $\Omega_{a}$ o conjunto de estados sem sucessores (nós sorvedouros). Então, define-se a Probabilidade Terminal como $P(t, x),(t, x) \in$ $\Omega_{A}$. A Distribuição de Probabilidade Terminal fornece uma medida conveniente de critério de investimento em uma estratégia de Stop.

Seja $\tau$ o tempo em um estado terminal (Tempo de Parada), então

$$
\mathbb{E}[\tau]=\Delta \sum_{(t, x) \in \Omega_{A}} t P(t, x)
$$

e

$$
\operatorname{Var}[\tau]=\Delta \sum_{(t, x) \in \Omega_{A}}(t-\mathbb{E}[\tau])^{2} P(t, x)
$$

Também é possível calcular a probabilidade de se atingir a barreira superior (Stop Gain) ou inferior (Stop Loss):

$$
\begin{aligned}
& P(\text { StopGain })=\sum_{\left(t, K_{t}\right) \in \Omega_{A}} P\left(t, K_{t}\right) \\
& P(\text { StopLoss })=\sum_{\left(t, L_{t}\right) \in \Omega_{A}} P\left(t, L_{t}\right)
\end{aligned}
$$

Para implementação computacional da recursão 8.2 é necessário definir uma distribuição para probabilidade de transição do preço do ativo. Uma premissa comum em modelagem de preços de ativos é que os mesmos seguem um Processo de Wiener e que $\mu$ e $\sigma$ são constantes. Neste caso, para $\Delta \rightarrow 0$, um Passeio Aleatório converge para um Processo de Wiener, como visto em (Huynh et al., 2011). Assim, uma possibilidade seria a utilização de uma Gaussiana como Distribuição de Probabilidade de Transição. 
Por sua vez, (Cox et al., 1979) apresenta um modelo de transição de preços em um modelo binomial. Esse foi o modelo utilizado por (Warburtona e Zhang, 2006). A seguir, detalhamos melhor essa maneira de precificação discreta.

\subsubsection{Aproximação Binomial de (Cox et al., 1979)}

Premissas do modelo de precificação:

(i) O preço do ativo aumenta a uma taxa $u$ com probabilidade $p$ e decresce a uma taxa $d$ com probabilidade $1-p$, onde $u>1$ e $d<1$ em um período $\Delta$

(ii) $\mathrm{O}$ ativo não paga dividendos

(iii) A taxa livre de risco ${ }^{1} r_{f}$ é positiva e constante com $d<r_{f}<u$.

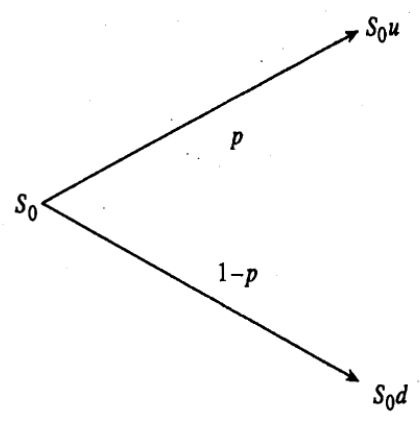

Figura 8.4: Transição no preço do ativo (Cox et al., 1979)

(Cox et al., 1979) mostram que, em uma hipótese de não-arbitragem ${ }^{2}$, temos que:

$$
\begin{gathered}
u=e^{\sigma \sqrt{\Delta}} \\
d=e^{-\sigma \sqrt{\Delta}} \\
p=\frac{e^{\mu \Delta}-d}{u-d}
\end{gathered}
$$

Com isso, em um modelo binomial com períodos de tamanho $\Delta / 2$ utilizamos as probabilidades de transição $p_{b}$ e $q_{b}$ dadas por $p_{b}=\left(e^{\mu \Delta / 2}-d_{\Delta / 2}\right) /\left(u_{\Delta / 2}-d_{\Delta / 2}\right), q_{b}=1-p_{b}$, onde $u_{\Delta / 2}=e^{\sigma \sqrt{\Delta / 2}}$, $d_{\Delta / 2}=1 / u_{\Delta / 2}$. Assim, notando que uma árvore trinomial pode ser construída com dois passos em uma árvore binomial, as probabilidades de transição do modelo trinomial são $p=p_{b}^{2}, q=q_{b}^{2}$, $r=2 p_{b} q_{b}$. Dessa forma, a cada passo o preço do ativo cresce por um fator $u$ ou decresce por um fator $d$, onde $u d=1$ e $u=u_{\Delta / 2}^{2}, d=1 / u$.

Passeios aleatórios para precificação de ativos têm sido estudados extensivamente. Veja (Cox et al., 1979), (Hull, 2012), (Huynh et al., 2011). Como visto, (Cox et al., 1979) fornece uma modelagem com probabilidades de transição constantes: assumindo que $K$ e $L$ são constantes, são derivadas expressões analíticas para $P(t, K)$ e $P(t, L)$. Contudo, o esforço computacional gasto para calcular essas expressões é no mínimo tão custoso quanto na implementação da recursão de (Zhang, 2001). Recursão essa também presente no modelo de (Warburtona e Zhang, 2006), como vimos.

A modelagem proposta por (Warburtona e Zhang, 2006) é de difícil implementação em uma plataforma de GPU devido à recursão sugerida em sua função de probabilidade proposta. Na verdade, a funcionalidade de recursão inexiste em placas gráficas de Compute Capability 1.x. A seguir, apresentamos um algoritmo alternativo para o cálculo de probabilidade de Stop com modelo trinomial

\footnotetext{
${ }^{1}$ Uma taxa de juro livre de risco é aquela que pode ser auferida sem que se assuma qualquer risco. Isso que dizer que se tivermos uma quantia $Q$ de reais, é garantido que teremos $Q e^{r_{f} T}$ daqui a $T$ anos se aplicarmos a uma taxa livre de risco $r_{f}$. No Brasil, essa taxa é geralmente relacionada aos títulos soberanos do Governo Federal.

${ }^{2}$ Grosso modo, arbitragem refere-se a uma situação onde se pode obter ganho sem risco.
} 
via Simulação Estocástica. A solução proposta tem paralelismo inerente e é de fácil portabilidade em GPU.

\subsubsection{Simulação Estocástica do Modelo Trinomial}

Como vimos, em um modelo trinomial temos três estados possíveis para transição de preços: subida, descida ou o ativo tem seu preço inalterado. Assim, considere o espaço de probabilidade $\Omega=\{$ Subida,Descida,Constante $\}$. Seja $X$ uma variável aleatória, tal que $X(\omega) \in\{u, 1, d\}$, onde $\omega$ pertence ao espaço $\Omega$. Então, pelo modelo de transição de preços de (Cox et al., 1979), definimos uma função de probabilidade $U_{3}(\omega)$ como:

$$
U_{3}(\omega)=\left\{\begin{array}{l}
P(\omega: X(\omega)=u)=p, \\
P(\omega: X(\omega)=1)=q, \\
P(\omega: X(\omega)=d)=r
\end{array}\right\}
$$

Assim, podemos construir o Algoritmo 4 utilizando a técnica de aceitação e rejeição para geração da distribuição de probabilidade $U_{3}(x)$ de transição de preços. Esse algoritmo é baseado no caso geral do método de aceitação e rejeição, cuja corretude pode ser obtida em (Glasserman, 2004).

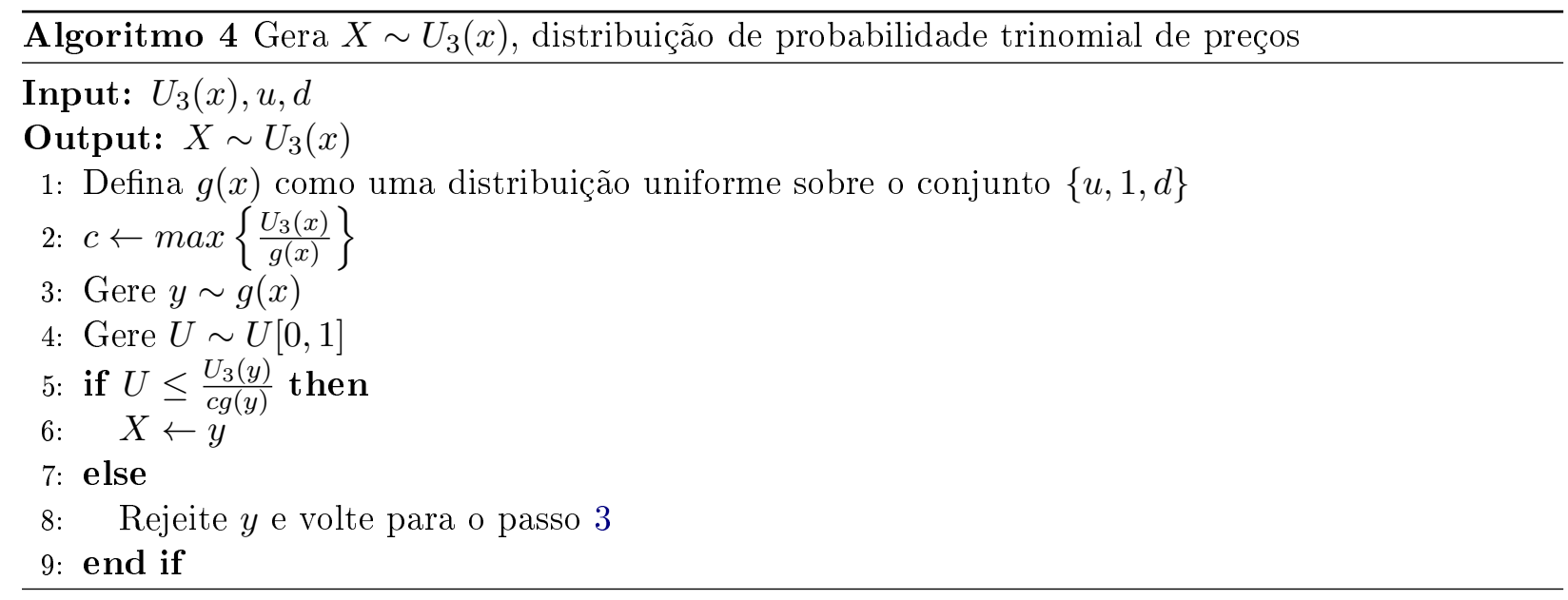

De posse do gerador $U_{3}(x)$, podemos simular um caminho pseudo-aleatório para o preço do ativo até que o horizonte de tempo limite seja alcançado ou a barreira de Stop superior seja atingida. Podemos repetir esse processo um número muito grande de vezes e, ao final, contar quantas vezes o Stop superior foi atingido e, assim, calcular a probabilidade de Stop Gain. O Algoritmo 5 apresenta esse algoritmo de Simulação Estocástica proposto em CUDA.

É fácil notar que o Algoritmo 5 proposto pode ser adaptado para cálculo de Stop Loss ao alterar a condição da linha 7 para comparação de barreira inferior (i.e. $S_{0} \leq L$ ).

\subsubsection{Notas sobre Stops Móveis}

Em contraste com Stops Fixos, ao usarmos Stops móveis, quando o preço de um ativo aumenta, o valor de Stop Loss configurado é atualizado. Nesta seção, apresentamos uma breve análise teórica do problema de Stops Móveis em um horizonte de tempo ilimitado.

Considere um ativo com preços movendo-se de acordo com o modelo trinomial visto, exceto pelo $T$ que deverá ser ilimitado. Sejam $K$ e $M=-L$ inteiros positivos que definem os valores de Stop Gain e Stop Loss, respectivamente. Se o preço cai a um valor $-M$ antes que $K$ seja atingido, a operação tem um Stop. Caso contrário, se o preço alcança o valor $K$ atualizamos o valor de Stop Loss para $K-M$. Assim, se o preço atinge a barreira superior $(j-1) K, j>1$, atualizamos o valor de Stop Loss para $(j-1) K-M$.

A probabilidade do ativo atingir uma barreira superior $K$ antes que atinja seu valor de Stop $M$ é dada pelo resultado clássico da Ruína do Apostador (Cover, 1987), no qual um apostador tem a 


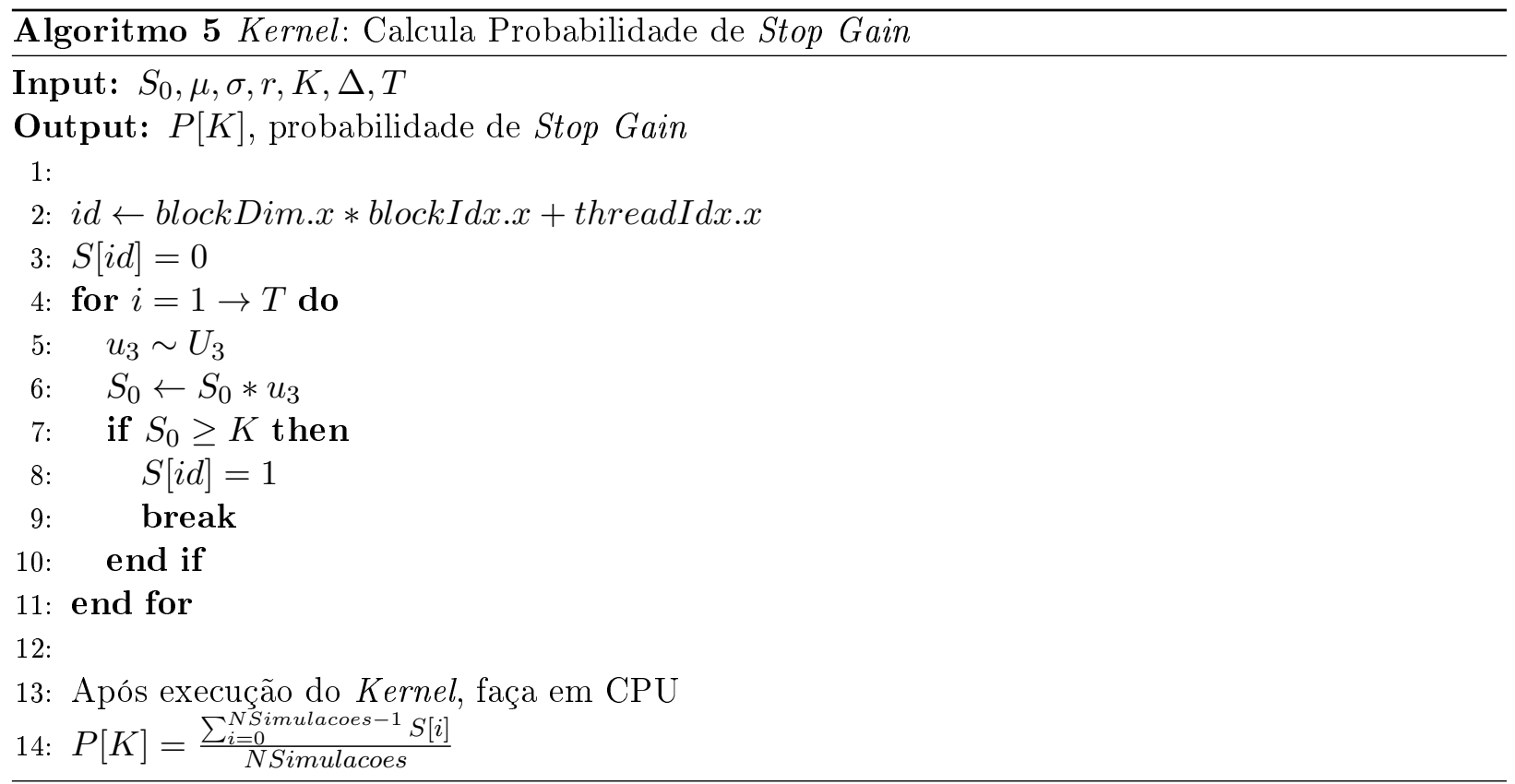

probabilidade $P$ de ganhar $K$ antes de perder $M$ :

$$
P=\frac{1-(q / p)^{M}}{1-(q / p)^{K+M}}
$$

Para que o valor de barreira $(j-1) K-M$ seja atingido, é necessário que o valor de Stop Gain tenha sido atingido $(j-1)$ vezes antes que o valor de Stop Loss seja alcançado. Assim, a probabilidade desse evento ocorrer é dada pela distribuição geométrica

$$
U(j)=P^{j-1}(1-P)
$$

Dessa forma, a esperança e variância da variável aleatória $X$ que conta o número de saltos que ocorrem antes que a estratégia sofra um Stop são, respectivamente,

$$
\begin{gathered}
\mathbb{E}[X]=1 /(1-P) \\
\operatorname{Var}[X]=P /(1-P)^{2}
\end{gathered}
$$

De posse da distribuição de probabilidade do número de saltos, chegamos a um valor esperado para o preço em caso de Stop

$$
K P /(1-P)-M
$$

onde sua variância é dada por

$$
K^{2} P /(1-P)^{2} .
$$

\subsection{Modelagem Estocástica}

Nas seções anteriores, calculamos a probabilidade do evento de Stop utilizando uma técnica de precificação baseada em não-arbitragem para descrição do passeio aleatório dos preços. Uma alternativa de modelagem muito comum em finanças é a suposição de um processo estocástico seguido pelos preços do ativo. Nesta seção, apresentaremos os passos gerais de uma modelagem desse tipo ao resolver, de modo alternativo, o problema de Stop visto até aqui com um modelo estocástico. Com esse modelo, apresentaremos um método para obtenção da esperança de ganho em uma estratégia de Stop Gain e, ao final, resolveremos o problema original de obtenção do Stop ótimo. 
Considere o processo

$$
d S=\mu S d t+\sigma S d W
$$

onde $\mu$ e $\sigma$ são constantes e $d W$ representa um processo de Wiener.

Seja

$$
Y=\ln S,
$$

pelo Lema de Itô, temos que

$$
d Y=\left(\frac{\partial Y}{\partial t}+\mu S \frac{\partial Y}{\partial S}+\frac{1}{2} \sigma^{2} S^{2} \frac{\partial^{2} Y}{\partial S^{2}}\right) d t+\sigma S \frac{\partial Y}{\partial S} d W .
$$

Como

$$
\frac{\partial Y}{\partial S}=\frac{1}{S}, \quad \frac{\partial^{2} Y}{\partial S^{2}}=-\frac{1}{S^{2}}, \quad \frac{\partial Y}{\partial t}=0,
$$

então,

$$
d Y=\left(\mu-\frac{\sigma^{2}}{2}\right) d t+\sigma d W .
$$

Como $\mu$ e $\sigma$ são constantes, essa equação indica que $Y=\ln S$ segue um processo de Wiener. O mesmo possui, então, média $\mu-\sigma^{2} / 2$ e variância com taxa constante $\sigma^{2}$.

O incremento em $\ln S$ de um tempo 0 a um tempo futuro $T$ segue, portanto, uma distribuição normal com média $\left(\mu-\sigma^{2} / 2\right) T$ e variância $\sigma^{2} T$. Isso significa que

$$
\ln S_{T}-\ln S_{0} \sim \mathcal{N}\left(\left(\mu-\sigma^{2} / 2\right) T, \sigma^{2} T\right) .
$$

$\mathrm{Ou}$

$$
\ln S_{T} \sim \mathcal{N}\left(\ln S_{0}+\left(\mu-\sigma^{2} / 2\right) T, \sigma^{2} T\right) .
$$

Processos com essas propriedades são de comum uso para descrever a dinâmica de ativos financeiros e podem ser caracterizados como Processos Log-Normais.

Assim, podemos re-escrever a equação 8.17 em função de $S$ como:

$$
\ln S_{T}=\ln S_{0}+\left(\mu-\sigma^{2} / 2\right) \Delta t+\sigma \Delta W
$$

e podemos chegar na seguinte recorrência geral para o valor do ativo objeto no tempo

$$
S_{i+1}=S_{i} e^{\left(\mu-\sigma^{2} / 2\right) \delta t+\sigma \sqrt{\delta t} z}, \quad z \sim \mathcal{N}(0,1) .
$$

Note que, se $S_{0}>0$, temos um processo no qual o valor do ativo objeto sempre assume valores positivos, o que é uma característica desejável em muitas situações reais.

Dessa forma, podemos estimar o valor esperado $(\mathbb{E}[S(K)])$ do ganho de um Stop Gain conforme kernel proposto no Algoritmo 6. Nesse algoritmo, o valor obtido na venda do ativo é descontada de uma taxa de juros $r$, relativo ao tempo de negociação. Com isso, como critério de parada, além do valor de Stop, também é considerado um valor $\epsilon$ limite para desconto de taxa de juros, a partir do qual o ganho seria, aproximadamente, nulo. 


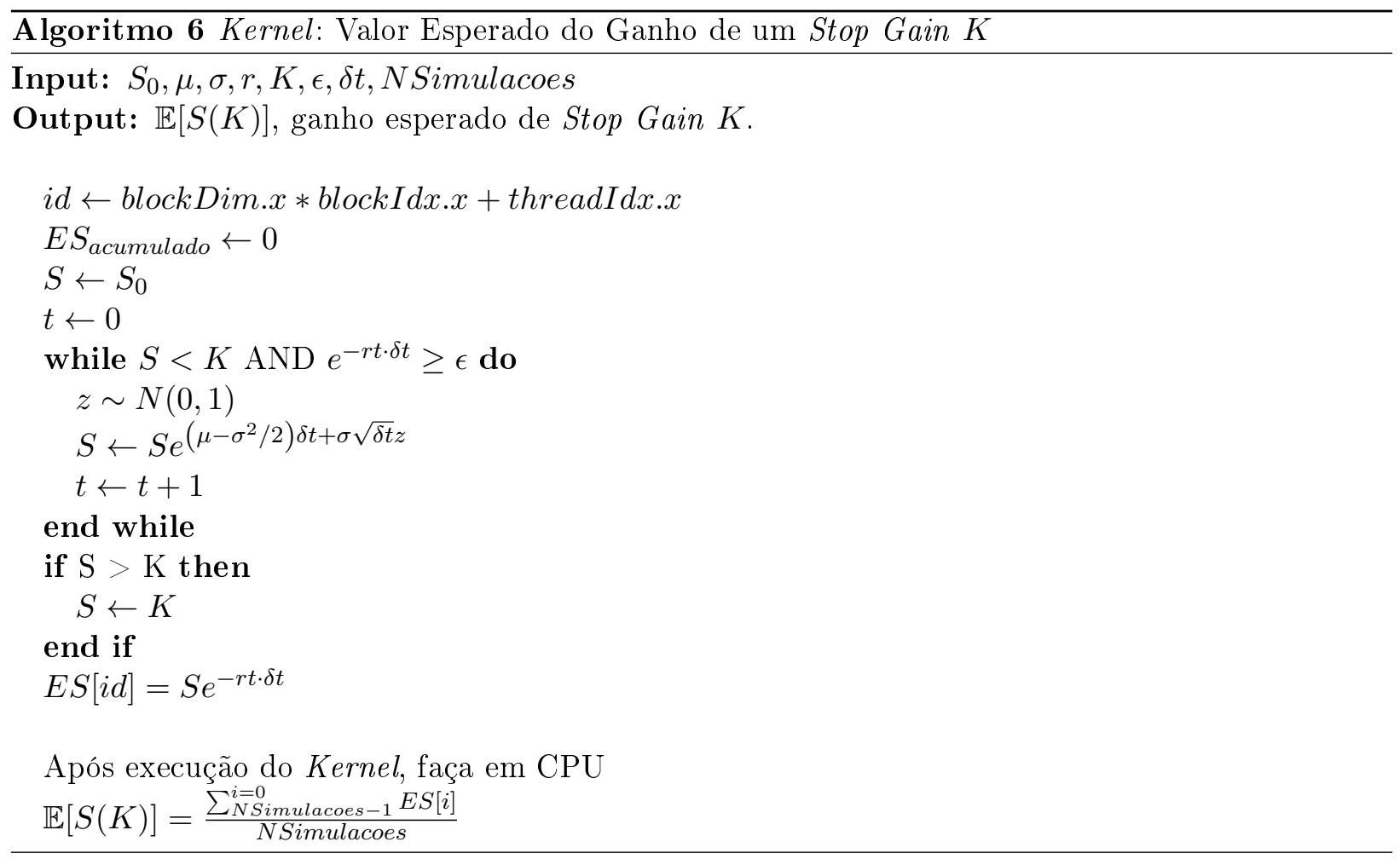

\subsubsection{Considerações Numéricas da Discretização de Euler}

$\mathrm{Na}$ seção anterior, encontramos a solução exata para o processo descrito na equação 8.16. Alternativamente, pela discretização de Euler, podemos chegar na seguinte recorrência:

$$
\begin{aligned}
S(t+\delta t) & =S(t)+\mu S(t) \delta t+\sigma S(t) \sqrt{\delta t} z \\
& =S(t)(1+\mu \delta t+\sigma \sqrt{\delta t} z), \quad z \sim \mathcal{N}(0,1) .
\end{aligned}
$$

Contudo, é importante notar que o número pseudo-aleatório $z$ pode ser gerado de uma distribuição Gaussiana em uma ou mais simulações de tal forma que

$$
(1+\mu \delta t+\sigma \sqrt{\delta t} z)<0
$$

ou seja,

$$
z<-\frac{1+\mu t}{\sigma \sqrt{\delta t}}
$$

e, assim, $S(t+\delta t)$ pode assumir valores negativos. Portanto, apesar da garantia de convergência para um processo log-normal, não há garantia que esse resultado seja obtido em todos os passos da simulação.

\subsection{Cálculo do Stop Ótimo}

Como vimos, de posse do Algoritmo 6, conseguimos obter um valor esperado de ganho $(\mathbb{E}[S(K)])$ dado um valor de Stop Gain $(K)$. Assim, podemos retomar nosso problema original de obtenção de Stop ótimo:

$$
\max \mathbb{E}^{x_{0}}\left[g\left(x_{\tau}\right)\right]
$$

sujeito a $\tau$ tempo de parada 
Seja $f(K)=\mathbb{E}[S(K)]$, então o valor $K=K^{*}$ tal que $f^{\prime}(K)=0$ é um ponto de extremo de Stop. Assim, a obtenção de Stop ótimo se reduz a um problema de obtenção de raiz de função, que pode ser resolvido, por exemplo, pelo método de otimização de bisecção áurea de Brent (W. H. Press e Flannery, 2007).

\subsection{Análise Experimental e Discussão}

Nesta seção realizamos uma análise experimental dos algoritmos de cálculo de probabilidade de Stop propostos no capítulo. Serão analisados os algoritmos propostos na seção 8.2.3 para o modelo trinominal em tempo discreto, o modelo estocástico da seção 8.3 e as implicações financeiras dos mesmos.

\subsubsection{Experimentos do Modelo Trinomial}

Os exemplos computacionais aqui apresentados possuem a seguinte configuração:

- Ativo objeto: Ação ITUB4 ${ }^{3}$

- Período de amostragem: 15/06/2011 a 31/05/2012

- $\mu:-0.1 \%$

- $\sigma: 19,98 \%$

- $r_{f}: 11 \%$

- $S(0,0): \mathrm{R} \$ 28,3$

- $\Delta: 2$

- $T: 20$

Antes de configurar um valor de Stop Loss, é de interesse do investidor saber qual é a probabilidade desse Stop acontecer. Utilizando o Algoritmo 5, a análise foi realizada variando a média e desvio padrão do ativo objeto em relação a diferentes configurações de preço de Stop Loss. A figura 8.5 exibe o comportamento da probabilidade de Stop Loss com o aumento da barreira inferior $(L)$. Nesse caso, o desvio padrão foi mantido fixo e houve variação na média. Como podemos perceber, com o aumento da média, a curva de probabilidade tem um aumento aproximadamente linear.

Na figura 8.6, o valor da média foi mantido fixo e variamos o desvio padrão. Também podemos concluir que quanto maior o desvio padrão maior a probabilidade de Stop Loss. Contudo, essa relação mostrou ser mais acentuada nesse último caso, em relação ao caso de variação da média.

Nas figuras 8.7 e 8.8, podemos observar os resultados obtidos em análise correspondente ao cálculo de Probabilidade de Stop Gain. Ao final, podemos concluir que quanto maior a média, menor a probabilidade de Stop Loss e maior a probabilidade de Stop Gain, mantida a variância fixa. Além disso, quanto maior o desvio padrão, maiores são as probabilidades de Stop Gain ou de Stop Loss, mantida a média fixa.

\footnotetext{
${ }^{3}$ Ação Preferencial Itaú Unibanco Holding S.A.
} 


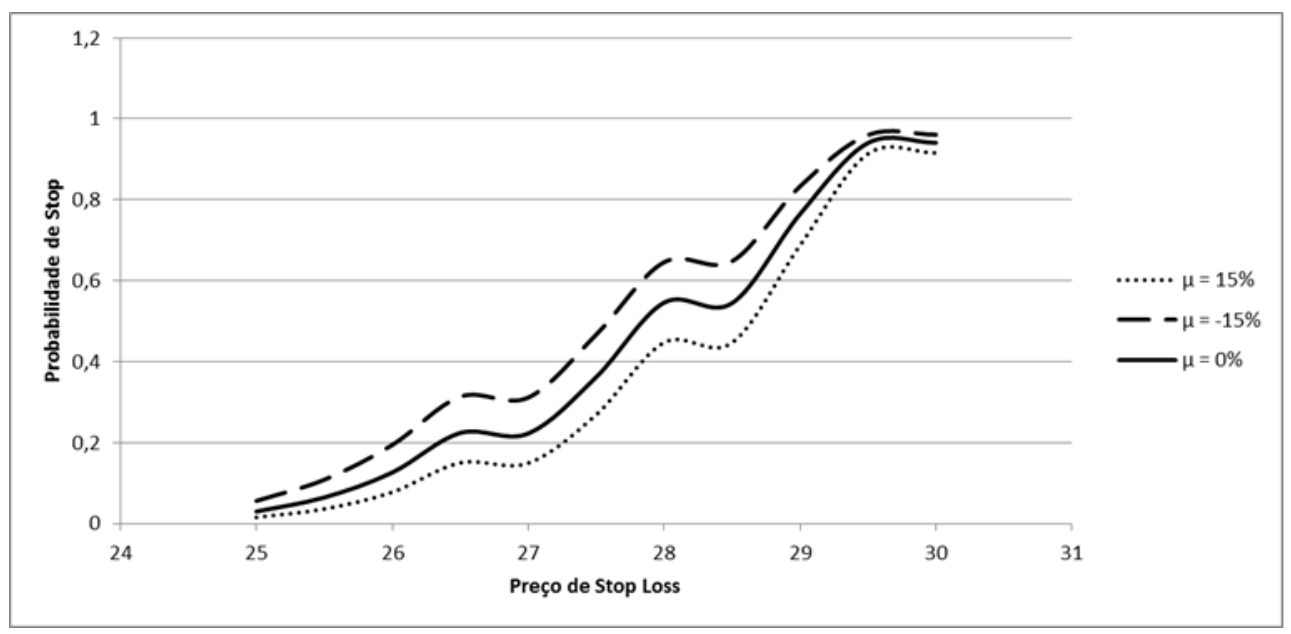

Figura 8.5: Probabilidade de Stop em função do preço de Stop Loss configurado. Valor do desvio padrão foi mantido fixo.

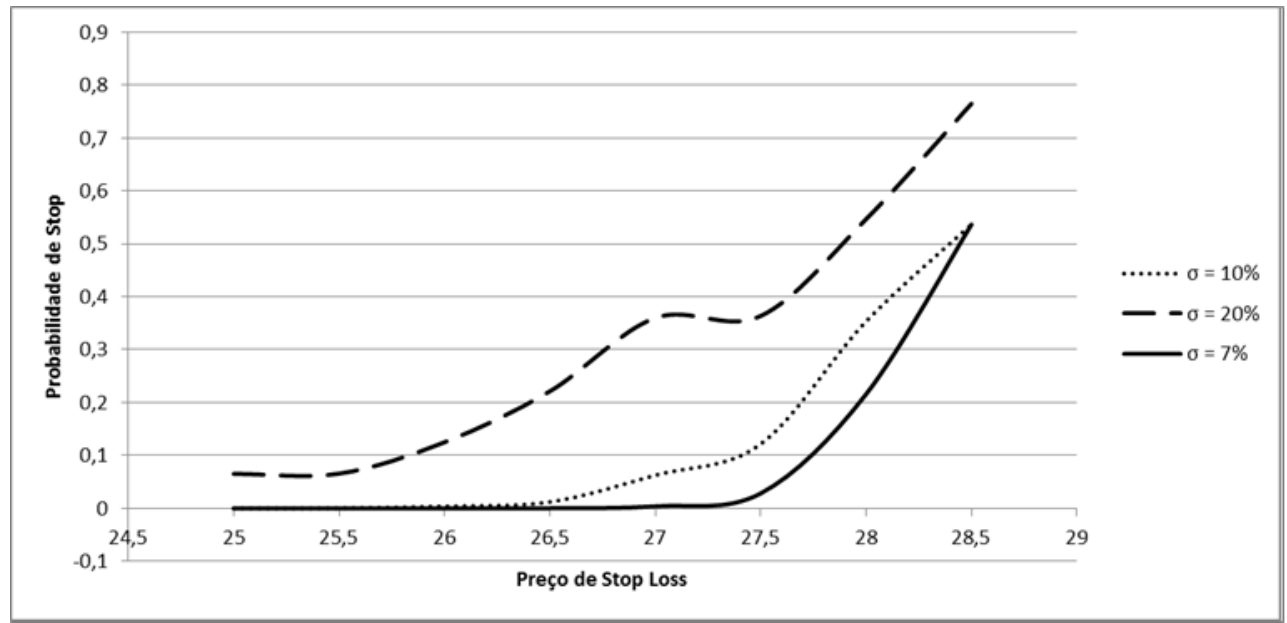

Figura 8.6: Probabilidade de Stop em função do preço de Stop Loss configurado. Valor da média foi mantido fixo.

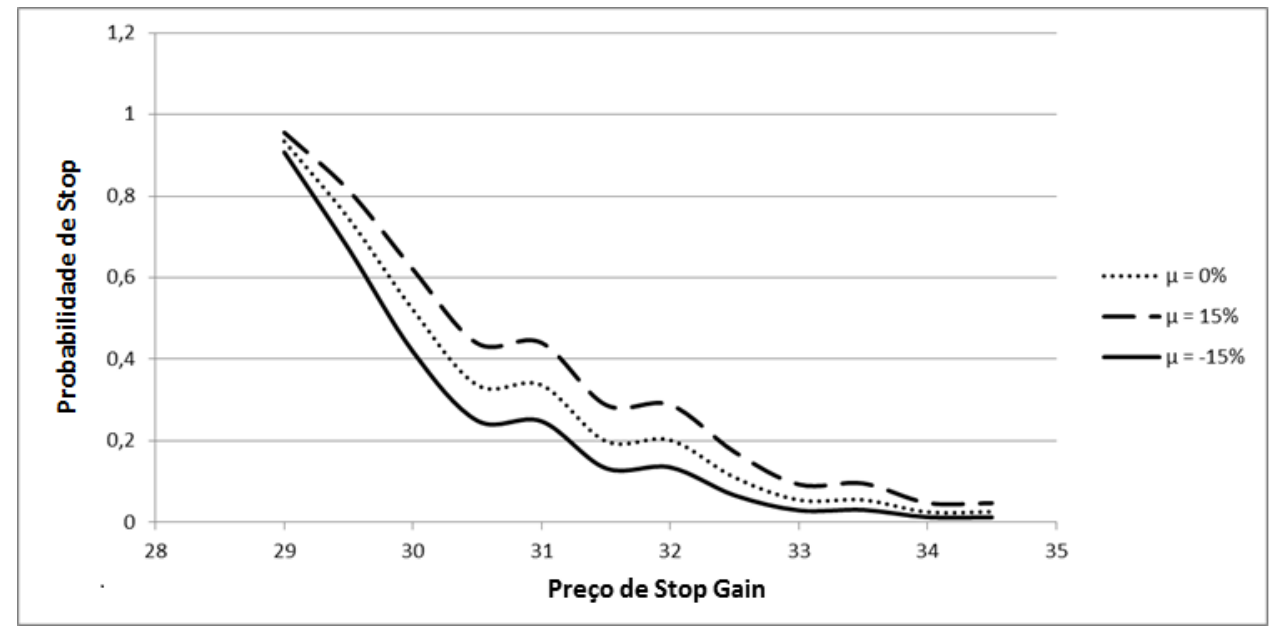

Figura 8.7: Probabilidade de Stop em função do preço de Stop Gain configurado. Valor do desvio padrão foi mantido fixo.

A modelagem proposta por (Warburtona e Zhang, 2006) é de difícil implementação em uma plataforma de GPU devido à recursão sugerida em sua função de probabilidade proposta. Enquanto 


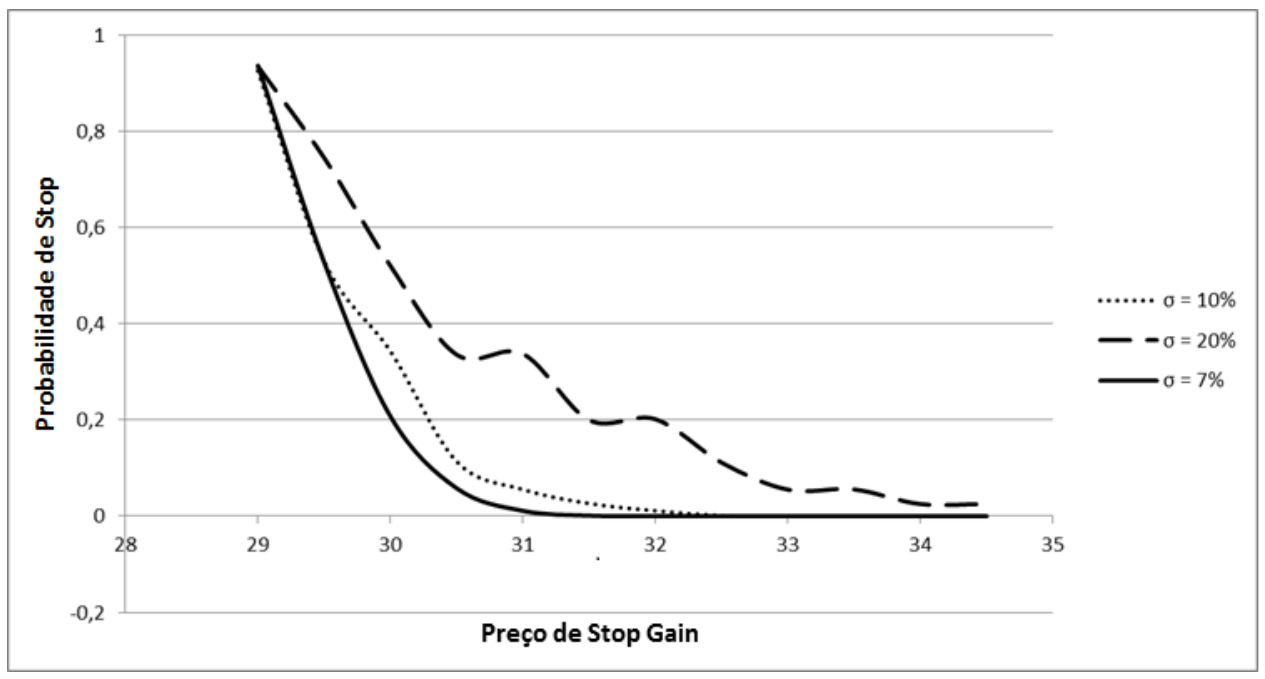

Figura 8.8: Probabilidade de Stop em função do preço de Stop Gain configurado. Valor da média foi mantido fixo.

isso, o algoritmo 5 proposto que resolve o problema via simulação estocástica pode ser facilmente portado em GPGPU. A figura 8.9 demonstra o tempo gasto nas implementações em CPU e GPU do algoritmo para cálculo de probabilidade de Stop Gain versus o valor da barreira configurado. O horizonte de tempo limite para simulação $(\Delta T)$ foi configurado em um valor de 2.000 dias. Como podemos ver, o tempo gasto na GPU mostrou ser estável ao longo de todas as simulações, enquanto que na CPU o tempo teve um acréscimo aproximadamente linear nas primeiras simulações. Isso mostra que o algoritmo paralelo é mais escalável. Para valores grandes de barreira o tempo gasto em CPU tendeu a se estabilizar. Isso é justificado pois para valores grandes de Stop Gain configurados, o fator limitante na simulação se torna o horizonte de tempo definido.

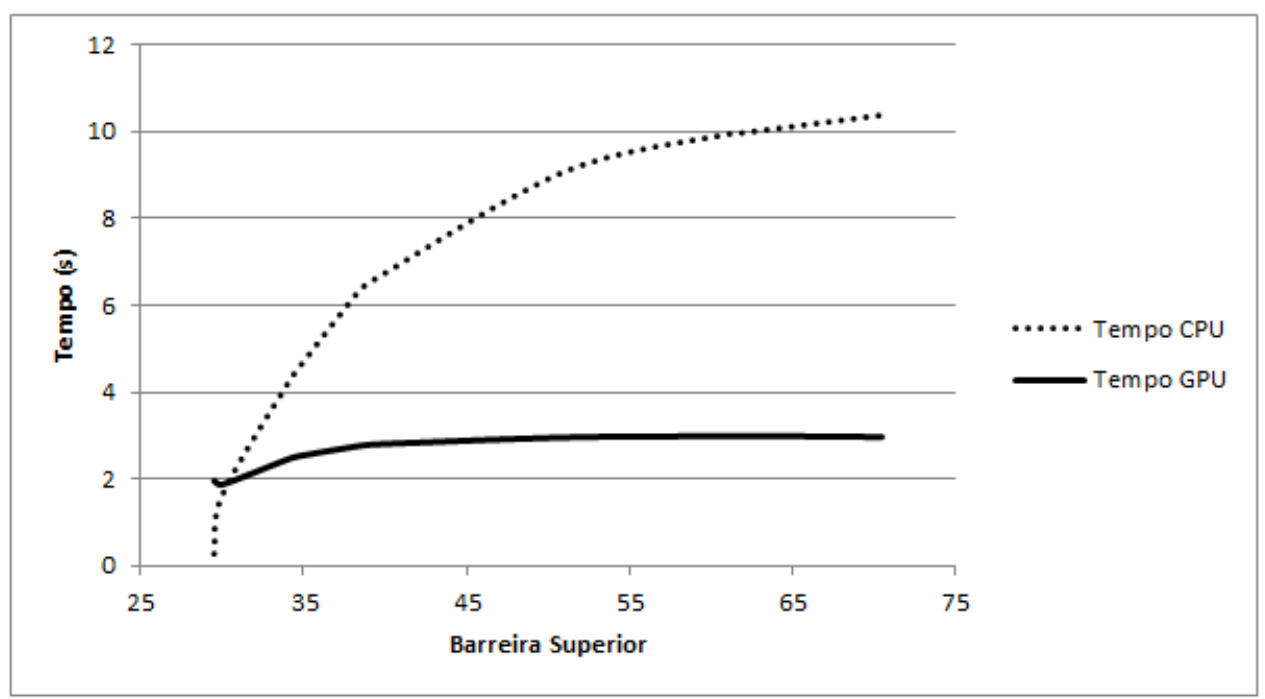

Figura 8.9: Tempo gasto para cálculo de probabilidade de Stop Gain em função do preço de barreira configurado.

\subsubsection{Experimentos do Modelo Estocástico}

Os exemplos computacionais aqui apresentados possuem a seguinte configuração:

- $\mu: 0,5 \%$ 
- $\sigma: 2,4432633 \%$

- $r_{f}: 13 \%$

- $S_{0}: \mathrm{R} \$ 33,99$

- Total de dias do passeio aleatório dos preços: 2520 (10 anos)

- Tamanho do passo temporal $(\delta t): 1$ dia

- Número de Simulações: 10.000

Nesta seção, apresentamos experimentos realizados com a modelagem estocástica e algoritmo propostos na seção 8.3. Assim, serão apresentados os resultados obtidos na obtenção da esperança de ganho em uma estratégia de Stop Gain.

Nesse modelo, há uma premissa que o preço do ativo analisado segue um processo log-normal e, assim, seu passeio aleatório pode ser iterado conforme equação 8.18. A figura 8.10 ilustra a simulação de diferentes passeios aleatórios para esse processo.

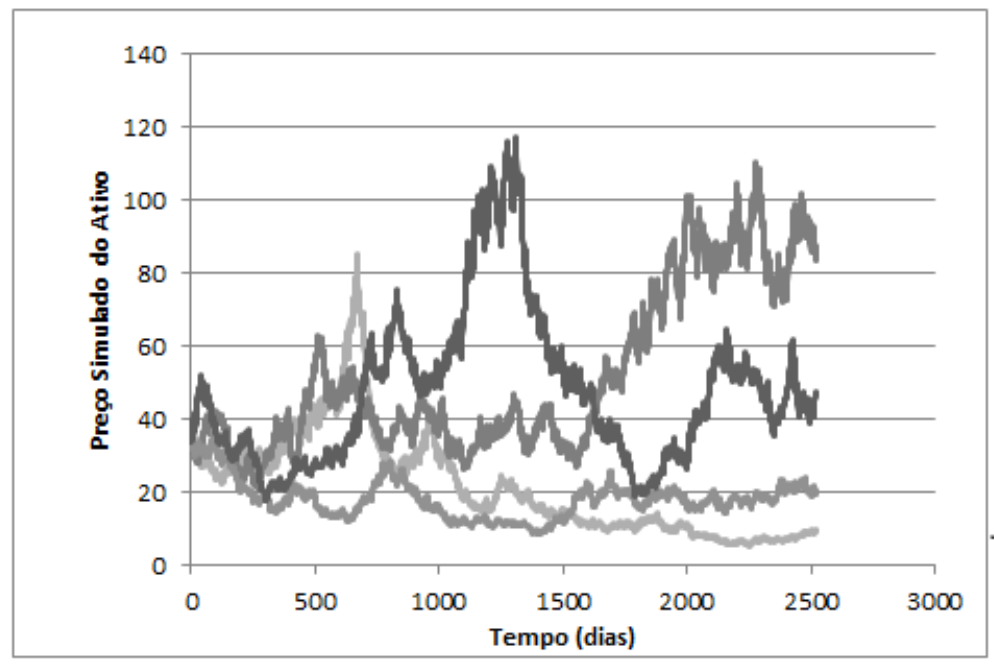

Figura 8.10: Simulação de passeios aleatórios do processo log-normal.

Por meio da simulação dos passeios aleatórios dos preços, é possível obter o ganho esperado de um Stop Gain configurado com a implementação do Algoritmo 6. As figuras 8.11 e 8.12 apresentam os resultados obtidos. Como podemos perceber, com o aumento da média $(\mu)$ temos uma esperança maior para o valor da barreira configurado. Em contraposição, um valor de volatilidade maior sugere a necessidade de configuração de um valor mais alto para valor de barreira.

De posse da esperança do retorno de um Stop Gain, é possível calcular o valor de retorno ótimo conforme discutido na seção 8.4. A figura 8.13 exibe os valores de retornos ótimos obtidos para diferentes valores de taxa de juros configurados utilizando o método de bisecç̧ão áurea de Brent. Podemos observar uma estimativa de ganho ótimo de $9,91 \%$ sobre uma taxa de juros de $11 \%$, na configuração analisada. Além disso, é possível concluir que existe um valor crítico de taxa de juros a partir da qual não faz mais sentido a utilização de uma estratégia de Stop Gain, pois o retorno ótimo é negativo. No exemplo considerado, esse valor foi de aproximadamente $13 \%$.

Com implementação do Algoritmo 6, também foi analisado o tempo computacional gasto na obtenção da esperança do ganho de um Stop Gain em GPU e CPU. Na figura 8.14. podemos ver o tempo gasto nesse cálculo em função do número de simulações realizadas. O tempo gasto em CPU apresentou um crescimento linear em relação ao número de simulações, contudo foi muito mais 


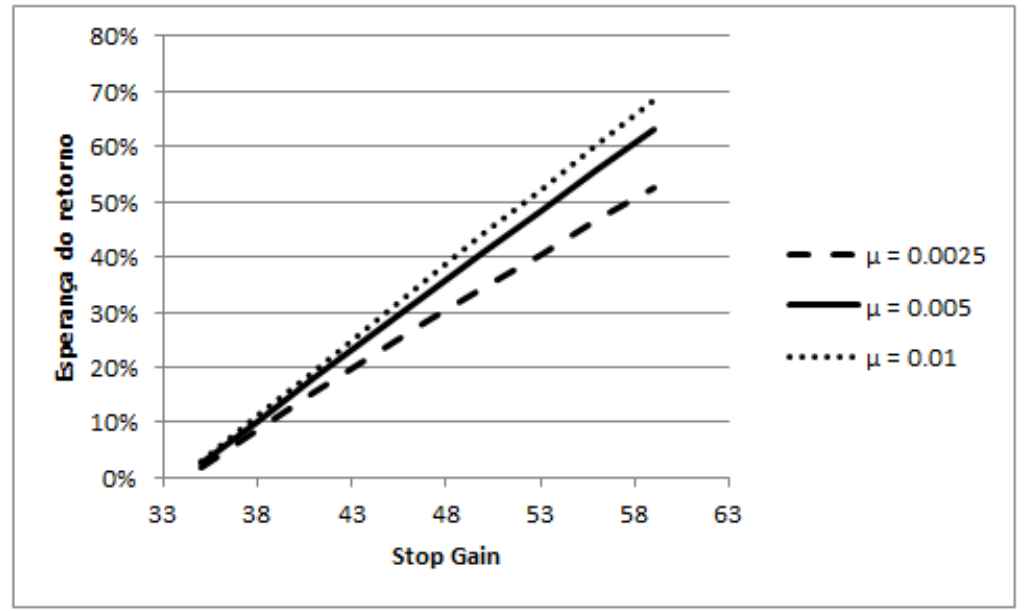

Figura 8.11: Esperança de retorno de Stop Gain. Análise da variação da média $\mu$.

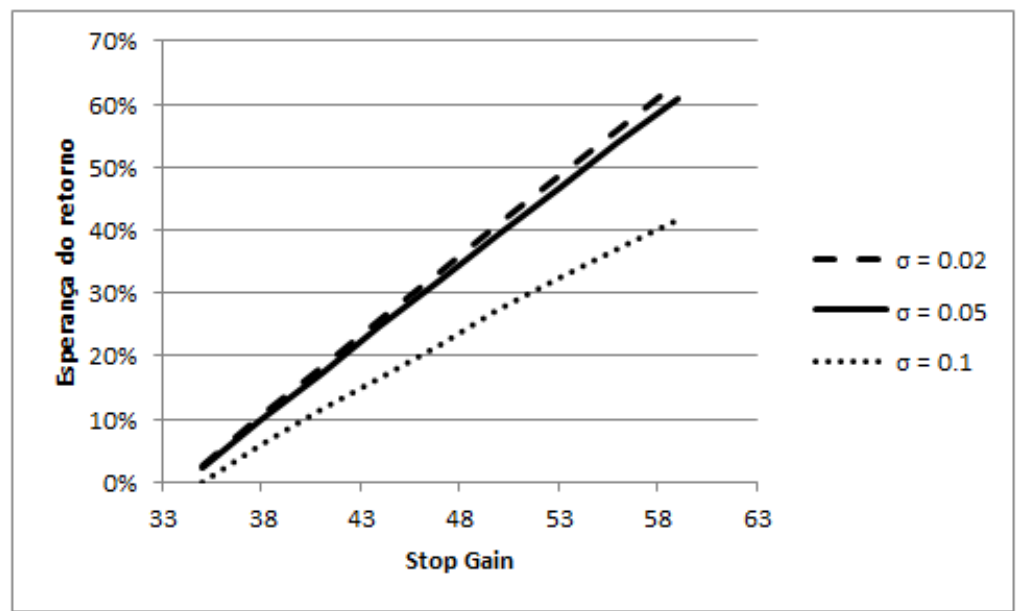

Figura 8.12: Esperança de retorno de Stop Gain. Análise da variação da volatilidade $\sigma$.

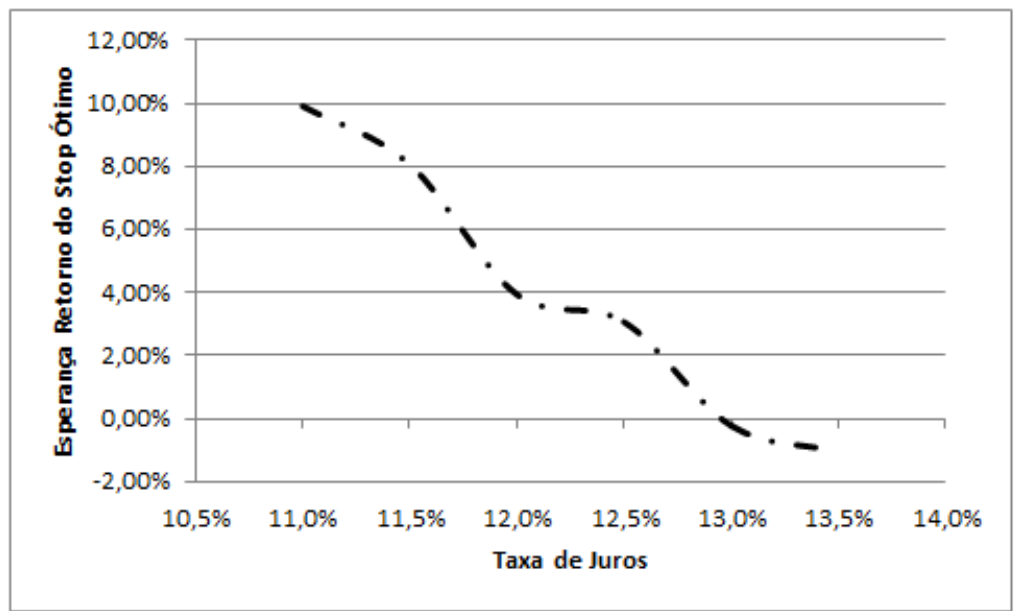

Figura 8.13: Esperança de retorno ótimo em função da taxa de juros. Configuração $\mu=0,0521139 \% e$ $\sigma=2,4432633 \%$. 
acentuado do que o crescimento no tempo gasto em GPU. Para um número de simulações igual a 51.200 foi obtido um speedup de mais de 12 vezes.

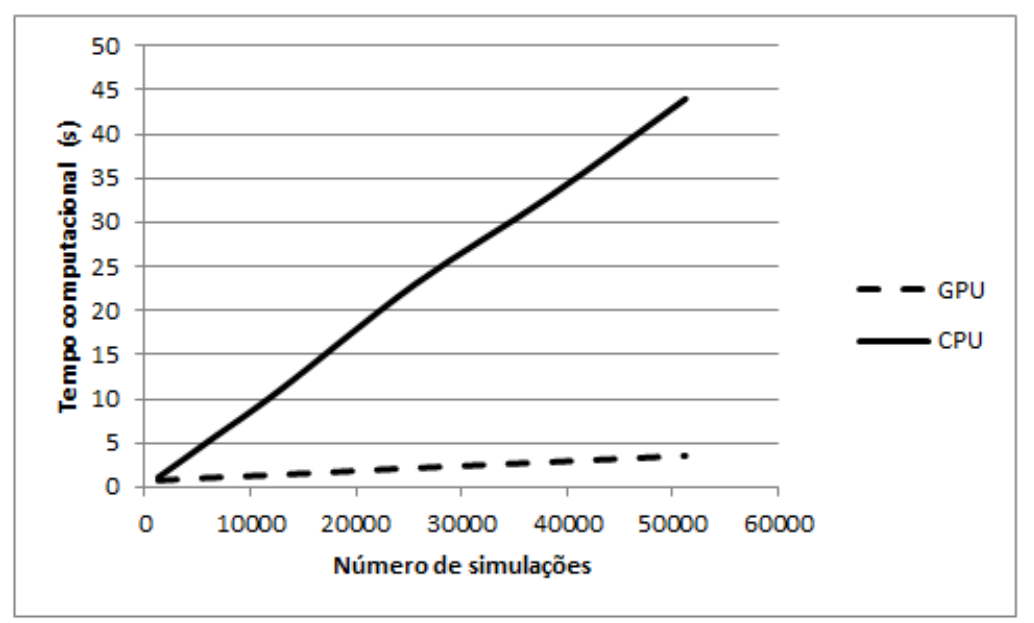

Figura 8.14: Tempo computacional gasto em cálculo da esperança de ganho de um Stop Gain em função do número de simulações. Configuração: Stop $=65, \mu=-0,00055 \%, \sigma=2 \%$.

A figura 8.15 apresenta o resultado obtido no tempo computacional variando o valor de Stop Gain configurado para um número fixo de simulações realizadas. Mais uma vez, o tempo computacional em GPU mostrou seu muito mais estável do que aquele medido em CPU. Para valores altos de barreira superior o tempo de cálculo gasto em CPU tendeu a se estabilizar. Isso é justificado pois para valores grandes de Stop Gain configurado, o fator limitante na simulação se torna o horizonte de tempo definido.

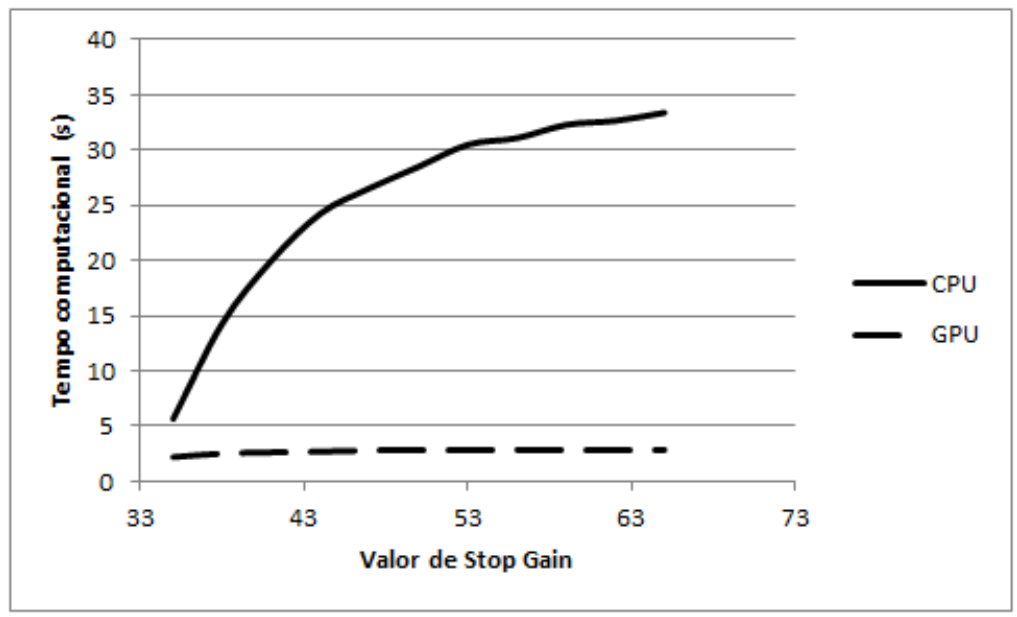

Figura 8.15: Tempo computacional gasto em cálculo da esperança de ganho de um Stop Gain. Configuração: número de simulações $=38.400, \mu=-0,00055 \%, \sigma=2 \%$. 


\subsection{Conclusão}

Neste capítulo realizamos o estudo de caso do problema de Stops Ótimos em finanças. Após definição formal do problema, revisamos o modelo trinomial proposto por (Warburtona e Zhang, 2006) e propusemos um algoritmo alternativo para o mesmo via simulação utilizando a técnica de aceitação e rejeição para probabilidade de transição dos preços. O algoritmo sugerido é, comparativamente, de fácil implementação e naturalmente paralelizável em GPU.

Em seguida, apresentamos uma modelagem estocástica para o problema e propomos um algoritmo para cálculo da esperança de ganho de um Stop Gain. De posse desse algoritmo, resolvemos o problema de otimização de Stop Ótimo.

Os algoritmos propostos foram implementados tanto em CPU quanto em GPU. Após análise financeira dos resultados, comparamos o tempo computacional gasto nas duas plataformas. Comparado com CPU, as implementações em GPU mostraram um gasto computacional muito mais escalável ao longo dos experimentos. Para o algoritmo de obtenção de esperança de retorno de um Stop Gain, foi alcançado um speedup de mais de 12 vezes.

\subsection{Notas e Leituras Complementares}

As origens do estudo da teoria de Stops remontam ao trabalho pioneiro de (Wald, 1945) sobre a natureza de experimentos estatísticos. Para uma introdução no assunto, veja (Karlin e Taylor, 1975), (Chow et al., 1971), (Oksendal, 1992). Uma abordagem em processos de Markov pode ser obtida em (Shiryaev e Aries, 2008). Um trabalho recente e aprofundado pode ser visto em (Gut, 2009). 


\section{Capítulo 9}

\section{Risco de Mercado}

Risco de Mercado é aquele relacionado com perdas no fluxo de caixa da organização causadas por flutuações nos preços de ativos e passivos da empresa (Kimura et al., 2010). Para gestão desse risco, uma métrica particularmente difundida é o Value-at-Risk (VaR), que visa a obter uma medida de perda máxima da instituição. Para o cálculo do mesmo, o gestor de risco deve ser capaz de calcular o valor de mercado de seus produtos, para assim conseguir avaliar as possíveis variações na carteira da empresa.

Com isso, neste capítulo abordaremos o cálculo de risco de mercado pela métrica de VaR. Com esse fim, estudaremos a precificação de um produto comum em negociações financeiras: Opções.

\subsection{Precificação de Opções}

Uma Opção é um produto financeiro que corresponde a um acordo entre duas partes, o comprador da opção e o vendedor da opção ${ }^{1}$. Nesse contrato, o primeiro tem o direito (mas não a obrigação) de exercer a opção acordada em um momento futuro, enquanto que o vendedor tem a obrigação de realizar a operação em caso de exercício pelo comprador. O valor pago pelo comprador é comumente chamado de prêmio da opção e corresponde ao seu preço de mercado. Há dois tipos básicos de opções. A opção de compra (call) dá ao seu detentor o direito de comprar um ativo a certo preço em uma determinada data. A opção de venda ( $p u t)$ dá ao seu detentor o direito de vender um ativo em uma certa data por determinado preço. A data especificada no contrato é conhecida como data de vencimento $^{2}$. O preço especificado no contrato é denominado preço de exercício (strike). As opções também podem ser classificadas conforme a data de exercício. Opções Europeias são aquelas que podem ser exercidas somente na data de vencimento. Já as Opções Americanas são mais flexíveis. Elas permitem o exercício em qualquer data até o vencimento.

A seguir, fornecemos um exemplo simples para entendimento da nomenclatura até aqui exposta.

\section{Exemplo 1. Opção de Compra de Dólar}

Suponha que uma Opção Europeia com vencimento em 24/12/2013 de Compra (Call) de Dólar à $R \$ 2,00$ em 24/12/2013 esteja sendo vendida a $R \$ 0,5$ hoje. Assim, um comprador deve pagar um prêmio de $R \$ 0,5$ para ter o direito de comprar a quantidade especificada de Dólar à $R \$ 2,00$ na data futura de 24/12/2013. Como se trata de uma opção europeia, o comprador pode ser exercer esse direito somente no vencimento, dia 24/12/2013.

Caso em 24/12/2013 o preço à vista (Spot) do dólar seja maior do que R\$2,00, o comprador pode lucrar ao exercer a opção de compra e vendê-la em seguida . Caso em 24/12/2013 o preço à vista (Spot) do dólar seja menor do que $R \$ 2,00$, o exercício da opção não trará lucro ao comprador.

Para ilustração, exibimos na figura 9.1 um trecho de um contrato de Opção de Compra de Taxa de Câmbio de Reais por Dólar Comercial disponível na BM\&FBovespa.

\footnotetext{
${ }^{1}$ também chamado de lançador da opção

${ }^{2}$ também conhecida como: data de expiração, data de exercício ou data de maturidade.

3 operação chamada de day-trade
} 


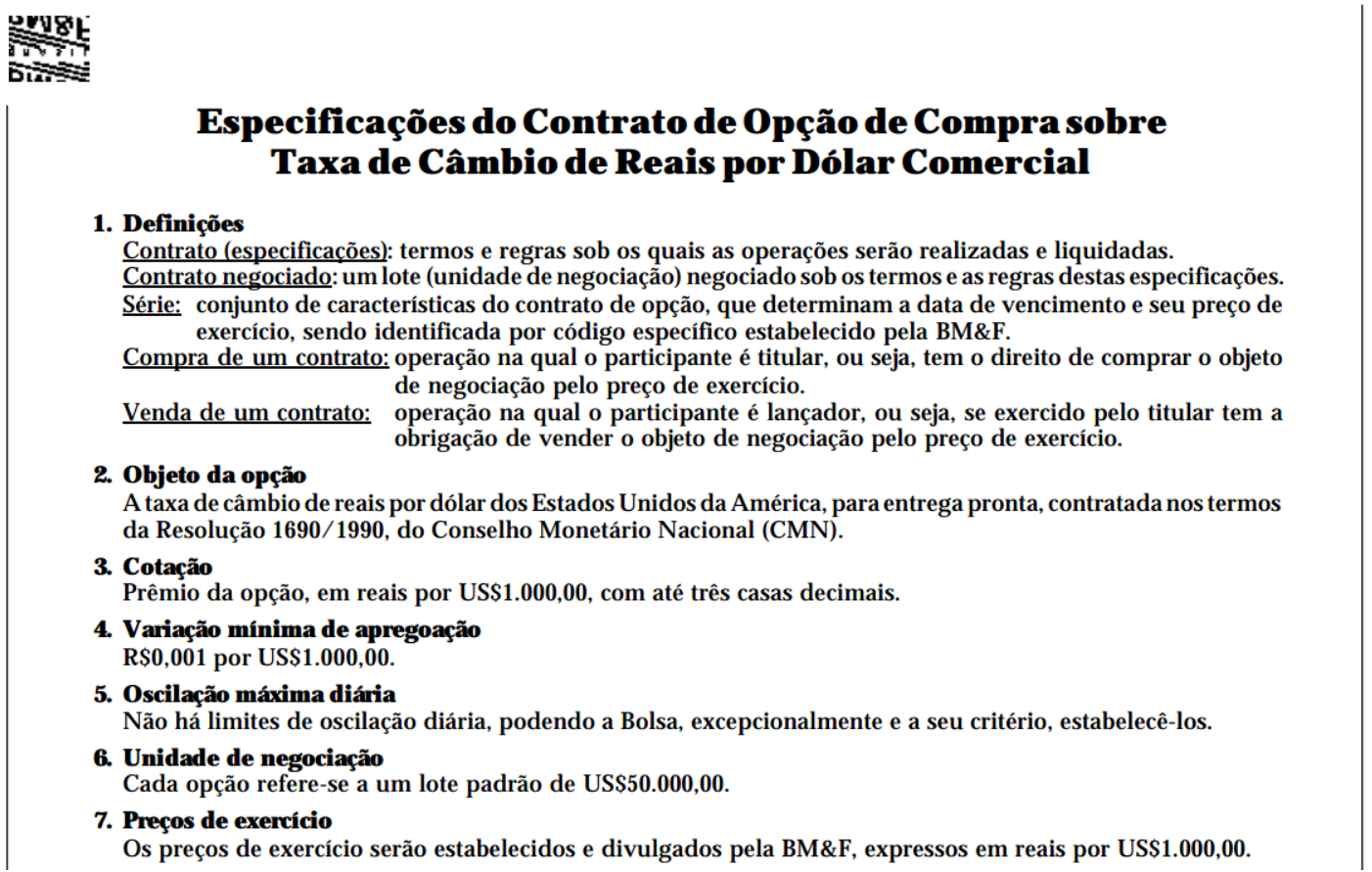

Figura 9.1: Contrato de Opção de Compra de Taxa de Câmbio de Reais por Dólar Comercial fornecido pela BMEFFBovespa

Assim, o problema de precificação de uma Opção se resume a encontrar seu preço justo (prêmio) a partir das variáveis de seu contrato de negociação como: Tipo da Opção, Data de Vencimento, Strike, Spot, taxa de juros livre de risco, volatilidade do ativo objeto etc. Nas próximas seções apresentamos os passos para precificação de uma opção via o método tradicional de Black \& Scholes em GPU, bem como, uma alternativa de precificação via simulação. Ao final, analisamos a possibilidade de melhoria na qualidade da simulação com utilização de técnicas de redução de variância.

\subsubsection{O Método Tradicional de Black-Scholes}

O método mais tradicional para precificação de opções é dado pelo modelo de Black \& Scholes (Black e Scholes, 1973). A partir da premissa de que os ativos seguem um movimento Browniano Geométrico, Black \& Scholes chegaram a uma expressão analítica de fácil utilização para obter o prêmio $V$ de uma opção Europeia dada pelas equações:

$$
\begin{aligned}
V & =S \times C N D\left(d_{1}\right)-X e^{-r T} \times C N D\left(d_{2}\right) \\
d_{1} & =\frac{\log \left(\frac{S}{X}\right)+T\left(r+\frac{v^{2}}{2}\right)}{v \sqrt{T}} \\
d_{2} & =d_{1}-v \sqrt{T}
\end{aligned}
$$

onde, $S$ é o valor Spot do ativo objeto, $X$ o Strike, $T$ o prazo para o vencimento, $r$ a taxa de juros livre de risco, $v$ representa a volatilidade do ativo objeto e $C N D$ é a distribuição normal padrão acumulada.

Para precificação de uma opção via equação 9.1, primeiramente necessitamos de um método numérico para cálculo da distribuição normal padrão acumulada:

$$
C N D(x)=\frac{1}{\sqrt{2 \pi}} \int_{-\infty}^{x} e^{\frac{-u^{2}}{2}} d u .
$$

Uma possibilidade é a aproximação por um polinômio de quinta ordem conforme sugere (Hull, 
2012). O código 9.1 implementa esse método em GPU.

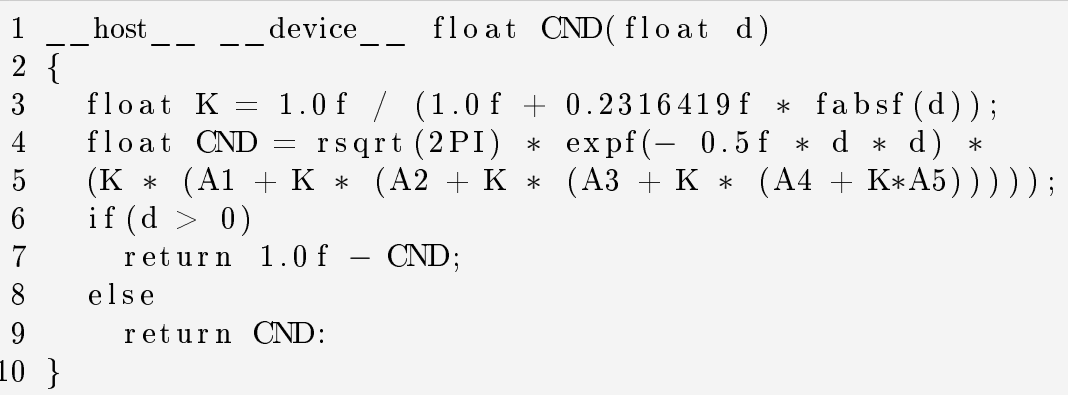

Código 9.1: Aproximação da distribuição normal acumulada padrão

$\mathrm{Na}$ Código 9.2, fornecemos os passos gerais para precificação paralela de um conjunto de $N$ opções em GPU. Esse passos podem ser sumarizados em:

1. Alocar vetores do host : hOptSpot(N), hOptStrike(N). Linhas 2 até 5.

2. Alocar vetores no device: $\operatorname{dOptSpot}(\mathrm{N}), \mathrm{dOptStrike(N)}$. Linhas 8 até 12 .

3. Inicializar vetores com variáveis dos contratos e mercado. Linha 14.

4. Transferir vetores da memória host para memória da GPU. Linhas 17 até 19.

5. Precificar opção em GPU via Black\&Scholes (Equação 9.1). Linha 22.

6. Transferir resultado da GPU para host. Linha 25.

7. Desalocar memória. Linhas 28 e 32 .

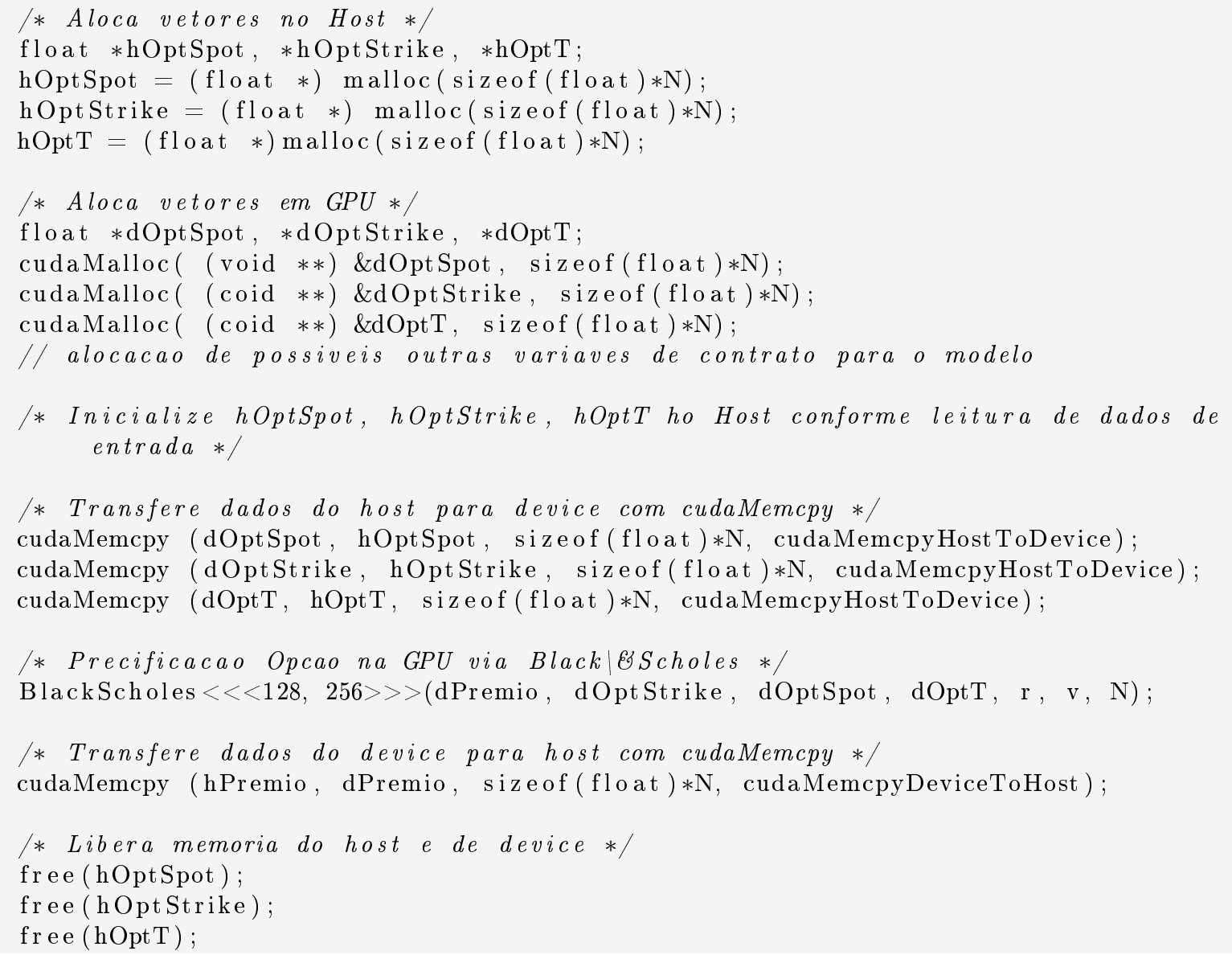


31

32

33

34

$35\}$

cudaFree (dOptSpot);

cudaFree (dOptStrike) ;

cudaFree (dOptT) ;

\subsubsection{Precificação via Simulação}

Como vimos, o método de Black \& Scholes fornece uma expressão de fácil uso para precificação de uma opção. Contudo, muitas vezes, não é possível obter uma expressão analítica para precificar esse produto financeiro. Assim, nesta seção, ilustramos uma forma de precificar uma opção via Simulação. Esse método pode ser utilizado independente da distribuição de probabilidade seguida pelo ativo objeto. O método aqui descrito supõe uma opção europeia, para precificação de outros tipos de opções via simulação de Monte Carlo veja (Jasra e Del Moral, 2011).

Antes de descrevermos o método de precificação via simulação, vamos analisar melhor a função de ganho (payoff) em uma negociação de contrato de opção europeia. Como sabemos, há quatro possibilidades de posições em opções:

1. Posição comprada em uma opção de Call

2. Posição comprada em uma opção de Put

3. Posição vendida em uma opção de Call

4. Posição vendida em uma opção de Put

É possível caracterizar essas posições em opções europeias em termos de payoff do investidor na data de vencimento. Seja $X$ o Strike e $S_{T}$, o preço final do ativo objeto, o resultado de uma posição comprada em uma opção de Call será:

$$
\max \left(S_{T}-X, 0\right)
$$

Já que a opção será exercida somente se $S_{T}>X$. Analogamente, temos que o payoff de uma posição vendida de uma opção de Call será:

$$
-\max \left(S_{T}-X, 0\right) .
$$

O payoff de uma posição comprada de uma opção de Put é:

$$
\max \left(X-S_{T}, 0\right)
$$

e o payoff de uma posição vendida de uma opção de Put é:

$$
-\max \left(X-S_{T}, 0\right) \text {. }
$$

A figura 9.2 ilustra essas quatro possibilidades de payoff.

Assim, o prêmio $\left(c_{T}\right)$ de uma Opção Europeia de Call pode ser calculado a partir de um valor esperado de seu retorno, conforme:

$$
c_{T}=e^{-r T} \mathbb{E}\left[\max \left(S_{T}-X, 0\right)\right] .
$$

Ao simular aleatoriamente o preço do instrumento $S_{T}$, podemos precificar a opção via simulação 

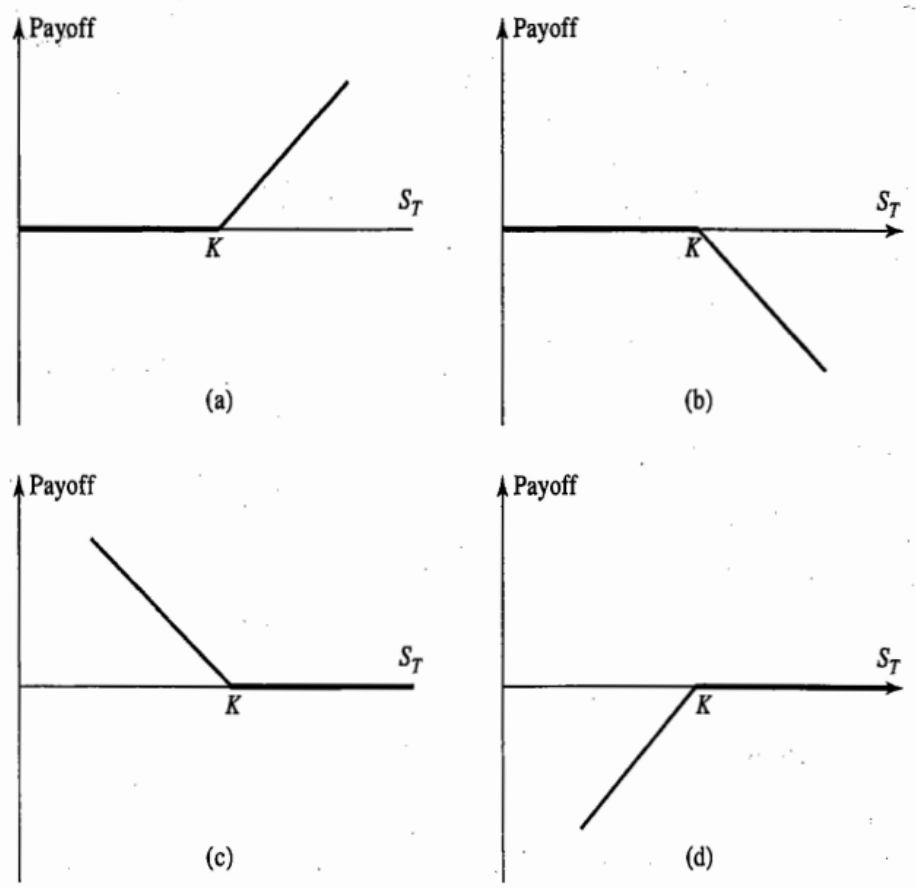

Figura 9.2: Payoffs das posições em opções europeias: (a) compra de Call; (b) venda de Call; (c) compra de Put; (d) venda de Put. Retirado de (Hull, 2012).

de monte carlo como

$$
\hat{c}_{T}=e^{-r T} \sum_{i=1}^{n} \max \left(S_{T}-X, 0\right) .
$$

A precificação de diferentes tipos de opções foi extensivamente estudada com aceleração em GPU (Solomon et al., 2010), (Pages e Wilbertz, 2010), (Pages e Wilbertz, 2012). Assim, de modo complementar, a seguir, iremos propor técnicas que visam à melhoria da qualidade do resultado obtido.

\section{Redução da Variância}

As equações vistas na seção anterior para precificação de opções call e put possuem uma aparente simetria. De fato, é possível provar que o valor de uma opção de call (c) pode ser obtida diretamente pelo valor da opção correspondente de put (p) (Hull, 2012):

$$
c+X e^{-r T}=p+S_{0}
$$

Essa relação é conhecida como paridade put-call. Ela mostra que o prêmio $c$ de uma opção europeia de call com strike $X$ e data de vencimento $T$ pode ser deduzido do valor da opção europeia de put com o mesmo preço de exercício e mesma data de exercício.

De posse da equação 9.9 , podemos estimar a esperança do prêmio de uma opção de call $\mathbb{E}[c]$ como:

$$
\mathbb{E}[c]=\mathbb{E}[p]+S-X e^{-r T}
$$

Em geral, a variância de uma opção de put é menor do que uma opção de call já que no primeiro caso o payoff é limitado, como podemos ver na figura 9.2. Dessa forma, o preço da opção de put se torna uma variável de controle para o cálculo do prêmio da opção de call e, assim, obtemos um estimador de $c$ com variância potencialmente reduzida.

A seguir, propomos o Algoritmo 7 para cálculo do valor esperado do prêmio de uma opção 
europeia de call baseado no procedimento de simulação sugerido na seção 9.1 .2 e na técnica de variável de controle proposta nesta seção. Além disso, também foi aplicada a técnica de variáveis antitéticas para melhoria da qualidade da simulação. Como podemos ver, nas linhas 6 e 7 as variáveis antitéticas são geradas, na linha 17 o valor esperado da opção europeia de put é calculado e, finalmente, o valor da opção de call é calculado na linha 18 pela relação de paridade put-call.

Algoritmo 7 Cálculo do valor esperado do prêmio de uma opção europeia de call via simulação utilizando técnicas de redução de variância.

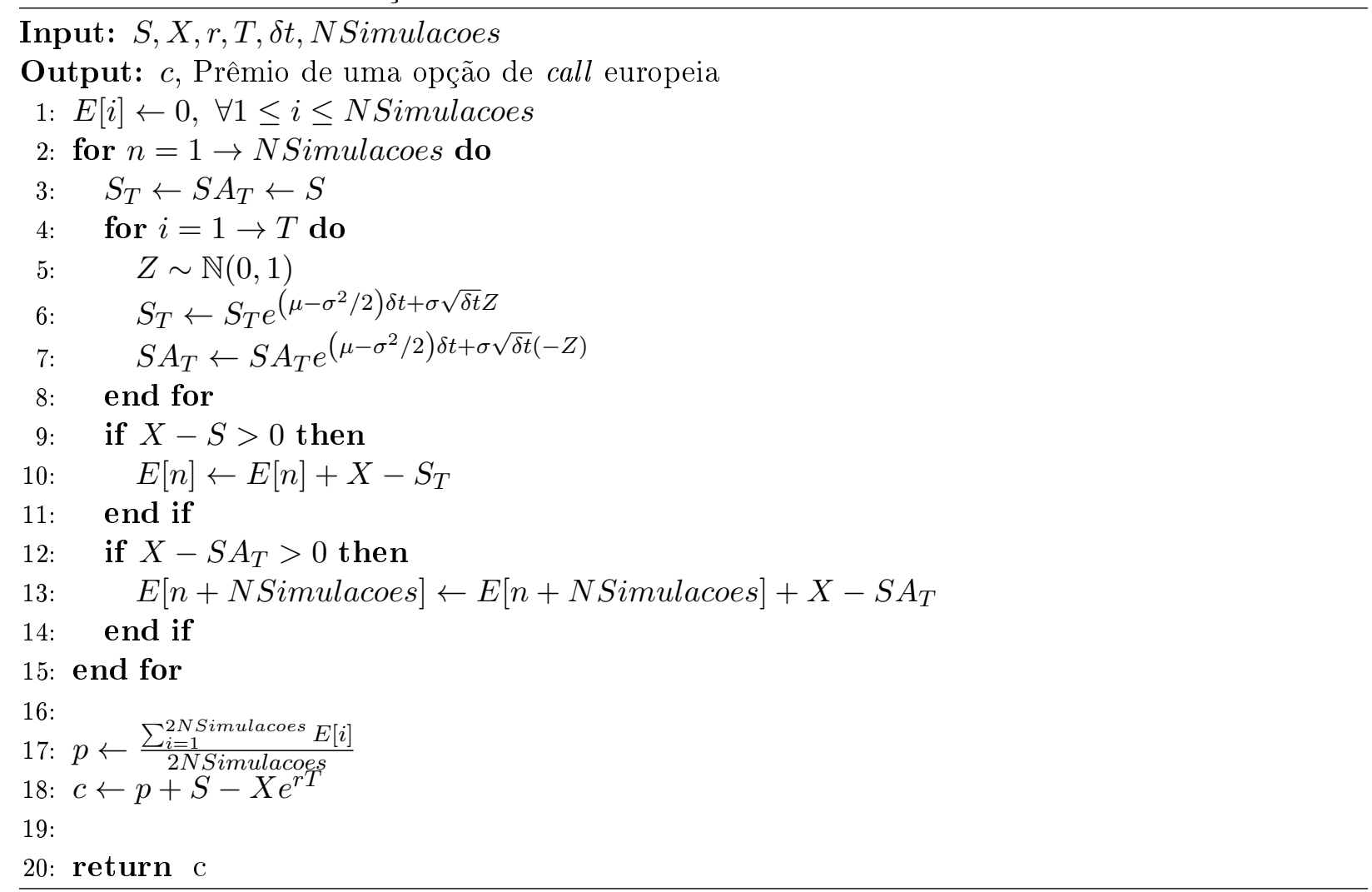

\section{$9.2 \quad$ Value-at-Risk}

(Choudhry e Tanna, 2007) define o $V a R_{1-\alpha}$ como a perda máxima que pode ocorrer em um grau de confiança $(1-\alpha)$ e período de tempo $(T)$ determinados. Um dos primeiros bancos a utilizar o conceito de VaR foi o J. P. Morgan. Em um de seus relatórios, o banco anunciou para carteira de sua tesouraria, um VaR de US\$ 15 milhões, para o horizonte de tempo de 1 dia e grau de confiança de 95\% (Kimura et al., 2010). Assim, podemos entender que o Banco forneceu uma previsão de que sua carteira não sofreria uma perda diária maior do que US\$ 15 milhões com uma probabilidade de $95 \%$. Uma grande vantagem do VaR é sua capacidade de resumir o risco de uma instituição financeira devido a múltiplas variáveis do mercado em uma única medida de fácil interpretação.

Se assumirmos que $F$ é a função de distribuição acumulada do retorno da carteira da instituição, podemos definir o VaR como

$$
F(V a R)=\alpha
$$

onde é $\alpha$ a probabilidade correspondente ao nível de confiança estabelecido. Ao utilizar a função de densidade dos retornos $f$, podemos definir o VaR de modo equivalente como

$$
\alpha=\int_{-\infty}^{V a R} f(x) d x .
$$


Assim, a questão principal no problema de análise de VaR está na obtenção da função $f$ de distribuição dos retornos para um horizonte de tempo definido. Para isso, existem três principais metodologias: Modelo Paramétrico, Simulação Histórica e Simulação de Monte Carlo.

Também chamado de Modelo de Variâncias-Covariâncias ou Delta-Normal, o modelo Paramétrico tem como hipótese a normalidade dos retornos. Dessa forma, a partir da média e do desviopadrão da variável aleatória associada ao retorno, pode-se construir facilmente os intervalos de confiança desejados.

O método de Simulação Histórica não necessita de estimação de parâmetros que reflitam uma distribuição de probabilidades, por isso, pode ser considerado um método não-paramétrico de estimação de VaR. Nesse tipo de simulação, a distribuição histórica dos retornos dos ativos da carteira é utilizada para simular os retornos futuros e, assim, o VaR desejado.

A ideia essencial do método via simulação de Monte Carlo é simular várias vezes um processo aleatório para as variáveis financeiras de interesse. Para isso, assume-se que as distribuições de probabilidade destas variáveis sejam conhecidas. Assim, variações hipotéticas de preços para os fatores de risco são criadas a partir de ocorrências aleatórias de um processo estocástico pré-determinado. De posse dessa amostragem, o cálculo do VaR é análogo ao método de simulação histórica. A figura 9.3 apresenta um resumo comparativo dos métodos enunciados.

\begin{tabular}{|c|c|c|c|}
\hline Itens & Delta-normal & Simulação Histórica & Simulação Monte Carlo \\
\hline \multicolumn{4}{|l|}{ Posições } \\
\hline Avaliação & Linear & Plena & Plena \\
\hline \multicolumn{4}{|l|}{ Distribuição } \\
\hline Formato & Normal & Corrente & Geral \\
\hline Varia no tempo & Sim & Possível & Sim \\
\hline Dados implícitos & Possível & Não & Possível \\
\hline Eventos extremos & Probabilidade baixa & Nos dados recentes & Possível \\
\hline Utiliza correlações & Sim & Sim & Sim \\
\hline Precisão do VaR & Excelente & Pobre quando a janela é pequena & Boa com muitas iterações \\
\hline \multicolumn{4}{|l|}{ Implementação } \\
\hline Facilidade de cálculo & Sim & Intermediária & Não \\
\hline Precisão & Depende da carteira & Sim & Sim \\
\hline Divulgação & Fácil & Fácil & Complicada \\
\hline Análise do VaR & Fácil, analítica & Mais difícil & Mais difícil \\
\hline Desvantagens & $\begin{array}{l}\text { Não-linearidades e } \\
\text { caudas pesadas }\end{array}$ & $\begin{array}{l}\text { Variação do risco ao longo do } \\
\text { tempo; Eventos extraordinários }\end{array}$ & Risco de Modelo \\
\hline
\end{tabular}

Figura 9.3: Resumo comparativo de metodologias de cálculo de VaR. Retirado de (Jorion, 2003)

A simulação de Monte Carlo pode amenizar alguns problemas da simulação histórica, como a não consideração da variação do risco no tempo e a janela temporal restrita utilizada. Além disso, tratase de método de maior flexibilidade por possibilitar a modelagem do comportamento dos preços por qualquer distribuição definida, ao contrário do método paramétrico, que supõe comportamento normal dos retornos. Contudo, o preço a pagar pela maior flexibilidade do método de Monte Carlo pode ser alto computacionalmente.

(Dixon et al., 2009) realizou experimentos de cálculo de VaR utilizando o método Delta-Normal em GPU e obteve speedup máximo de aproximadamente 8 vezes em uma implementação tradicional desse método. Alternativamente, nas próximas seções iremos explorar o método de Monte Carlo para cálculo de VaR e avaliar, experimentalmente, sua viabilidade em GPU. 


\subsubsection{Cálculo do VaR via Simulação de Monte Carlo}

Seja $\Pi_{0}=\left(S_{0}^{1}, S_{0}^{2}, \ldots, S_{0}^{n}\right)$ o valor de um portfólio composto por $n$ ativos em $t_{0}$. Desejamos calcular o $V a R_{1-\alpha}$ de $\Pi_{0}$ para um período $T$. A seguir, apresentamos os passos para resolução desse problema via Simulação de Monte Carlo:

1. Escolha e configure um modelo estocástico para projeção dos preços dos ativos do portfólio

2. Simule os preços dos ativos para projetar seus valores em $T: S_{T}^{1}, S_{T}^{2}, \ldots, S_{T}^{n}$

3. Calcule o valor do portfólio $\Pi_{T}=\left(S_{T}^{1}, S_{T}^{2}, \ldots, S_{T}^{n}\right)$

4. Repita os passos 2 e 3 um número $(N)$ de vezes tão grande quanto se queira

5. Ordene de modo crescente os valores dos portfólios obtidos e obtenha a distribuição: $\left(\Pi_{T}^{1}, \Pi_{T}^{2}, \ldots, \Pi_{T}^{N}\right)$

6. Compute o VaR a partir do quantil $(1-\alpha)$ de interesse: $V a R_{1-\alpha}=\Pi_{T}^{\lfloor(1-\alpha) N\rfloor}-\Pi_{0}$

O cálculo do valor de um portfólio pode ser de alto custo computacional (e.g. portfólio de opções). É fácil notar que as estimativas dos valores dos $N$ portfólios são independentes entre si e, portanto, podem ser facilmente calculadas de modo paralelo. Essas características sugerem que o cálculo de VaR seja suscetível em GPU. Dessa forma, no Algoritmo 8, propomos uma implementação desse método em CUDA.

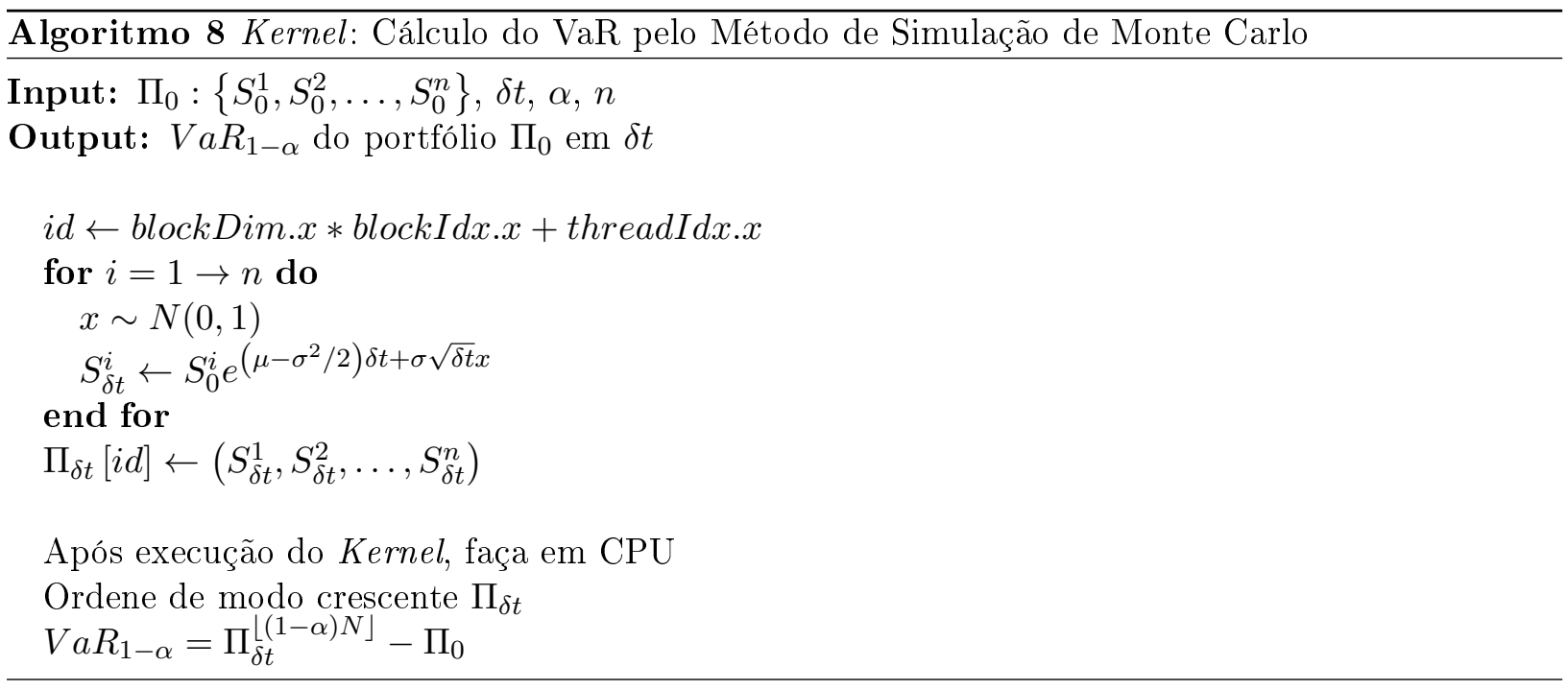

\subsubsection{Notas sobre Cálculo do VaR para Múltiplos Portfólios}

Considere $n$ portfólios $\left(\Pi^{1}, \Pi^{2}, \ldots, \Pi^{n}\right)$ com respectivos valores de VaR dados pelo vetor $x=$ $\left(V a R^{1}, V a R^{2}, \ldots, V a R^{n}\right)$. Então, o $V a R_{\Pi}$ do portfólio composto $\Pi=\left(\Pi^{1}, \Pi^{2}, \ldots, \Pi^{n}\right)$ é dado por

$$
V a R=\sqrt{x R x^{\prime}}
$$

onde, $R$ é uma matriz com as correlações dos portfólios:

$$
R=\left(\begin{array}{cccc}
1 & p_{12} & \cdots & p_{1 n} \\
p_{21} & 1 & \cdots & p_{2 n} \\
\vdots & \vdots & \ddots & \vdots \\
p_{n 1} & p_{n 2} & \cdots & 1
\end{array}\right)
$$


Em geral, a matriz $R$ pode ter grande dimensão. Assim, é comum a utilização de técnica de Análise de Componentes Principais para redução de dimensionalidade. Para uma implementação desse método em GPU veja (Jung e O'Leary, 2006).

É importante notar que cada portfólio pode ser precificado de uma maneira diferente. Por exemplo, $\Pi_{0}$ pode representar um portfólio de ações, $\Pi_{1}$ uma carteira de opções etc. Dessa forma, a execução de um único kernel em GPU para cálculo do VaR para múltiplos portfólios não é uma boa estratégia, na prática. Assim, de modo geral, é necessário construir um kernel para cada precificação realizada. Em placas de compute capability 2.x é possível o lançamento de kernels paralelos. Com isso, uma possível solução para esse problema seria a precificação de portfólios distintos em kernels de diferentes streams, conforme discutido na seção 4.2.2. Contudo, essa estratégia é limitada a um total de 16 kernels em paralelo em uma arquitetura Fermi, conforme visto no Apêndice A.1.

\subsection{Análise Experimental e Discussão}

\subsubsection{Experimentos da Precificação de Opções}

Para o cálculo das opções, foram utilizados as seguintes configurações:

- $\sigma: 25 \%$

- $r_{f}: 10 \%$

- Dias úteis até o vencimento: 126

- $S=K=25$

Visando à análise da qualidade da simulação proposta no Algoritmo 7 para cálculo do prêmio de uma opção de call europeia, o mesmo foi implementado em CPU com diferentes configurações.

Em primeira análise, o algoritmo foi testado sem aplicação de técnicas de redução de variância. Nessa configuração, a figura 9.4 apresenta o erro padrão obtido em simulações realizadas com três RNGs distintos: Mersenne Twister, Sobol e rand(), função padrão da biblioteca de C. Como podemos notar, de modo geral, o gerador Sobol apresentou uma qualidade maior. Esse resultado vai ao encontro do trabalho de (Giles et al., 2008), que sugere a utilização de QRNGs para simulações de Monte Carlo em aplicações financeiras.

Tendo em vista as técnicas de redução de variância sugeridas no capítulo, a figura 9.5 apresenta o erro padrão obtido utilizando as técnicas de variáveis antitéticas e variável de controle em Sobol. Podemos ver uma melhora na qualidade da simulação em ambos os casos, havendo um destaque para a última técnica. Ao utilizar as duas técnicas combinadas, o erro padrão da simulação apresentou uma melhora de mais de 4 vezes. 


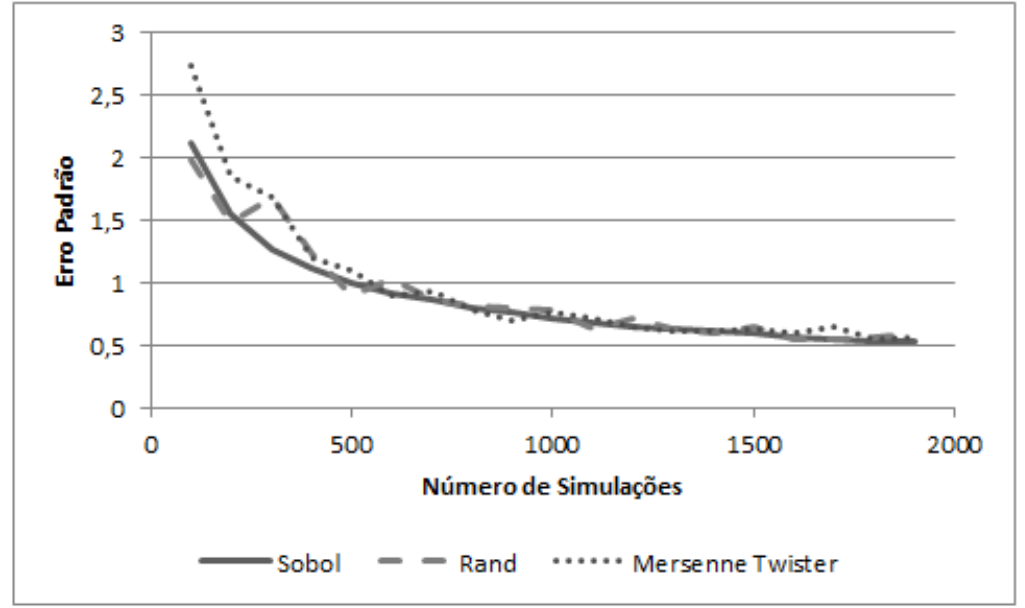

Figura 9.4: Simulação do prêmio de uma opção de call europeia em diferentes RNGs.

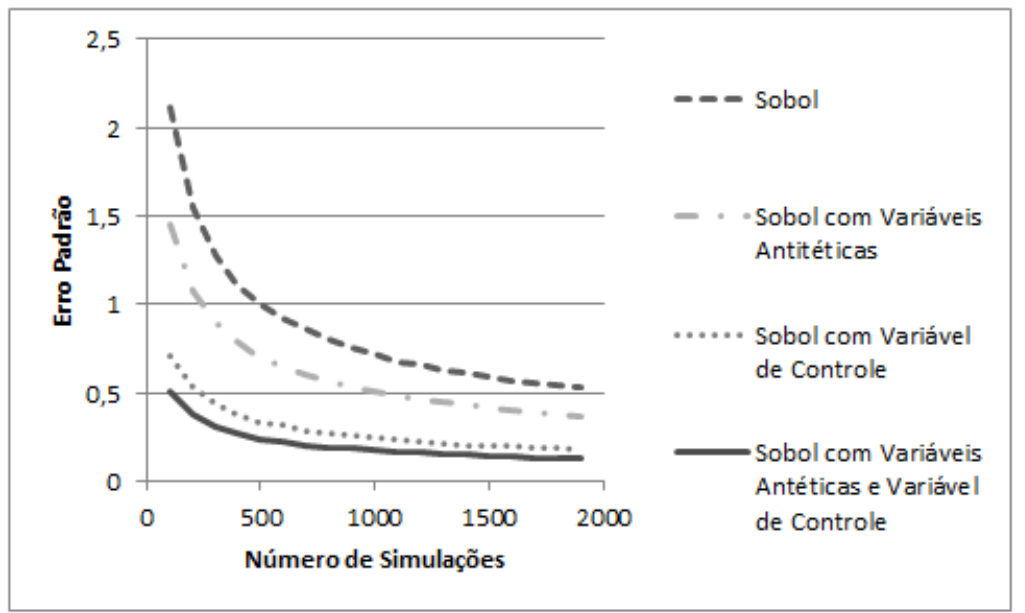

Figura 9.5: Aplicação de técnicas de redução de variância na simulação do prêmio de uma opção de call europeia utilizando Sobol como RNG.

\subsubsection{Experimentos do Cálculo de VaR}

Nesta seção, realizamos uma análise experimental do algoritmo de cálculo de VaR estudado nesse capítulo. Em vista à exploração de capacidade computacional, todos os experimentos foram realizados supondo o cálculo de VaR em um portfólio $\Pi_{0}$ de Opções Europeias. As mesmas foram precificadas conforme método de Black \& Scholes visto na seção 9.1.1. O VaR foi estimado a um nível de confiança de $95 \%$ para um período de 1 dia.

Para simulação dos preços dos ativos foram utilizados os seguintes parâmetros:

- $\mu:-0,05 \%$

- $\sigma: 2 \%$

Para o cálculo das opções foram utilizados as seguintes configurações:

- $\sigma: 25 \%$

- $r_{f}: 10 \%$

- Dias úteis até o vencimento: 126 
- A $i$-ésima opção do portfólio possui valores de Spot e Strike iguais a $i$.

O Algoritmo 8 foi implementado tanto em CPU quanto em GPU em vista à obtenção do $V a R_{95 \%}$ de 1 dia da carteira $\Pi_{0}$ gerada. O portfólio $\Pi_{0}$ foi gerado inicialmente com um total de 2 mil opções, o que resultou em um valor total de $\mathrm{R} \$ 191.740$. Nessa configuração, o valor obtido para o $V a R_{95 \%}$ de 1 dia foi de aproximadamente $-\mathrm{R} \$ 896$. Isso quer dizer que a carteira gerada tem uma previsão de perda máxima de $\mathrm{R} \$ 896$ em 1 dia a um nível de confiança de $95 \%$.

Nas figuras 9.6, 9.7 e 9.8 podemos observar a distribuição da variação de 1 dia no valor das carteiras simuladas. Na figura 9.6 apresentamos o resultado para um total de 128 simulações, enquanto que na figura 9.7 há um total de 1280 simulações. Como podemos notar, quanto maior o número de simulações, mais próximo o resultado de uma distribuição normal, o que fica evidente na figura 9.8 que corresponde a um número grande de simulações de 51.200.

Analise de tempo computacional foi feita variando o número de simulações e o número total de opções no portfólio. Nas tabelas 9.1 e 9.2 podemos observar o tempo computacional gasto em CPU e GPU, enquanto que nas figuras 9.9 e 9.10 são exibidos os speedups obtidos. Para um total de 2.500 opções com 51.200 simulações, foi obtido um speedup de mais de 50 vezes, como podemos observar na figura 9.10 .

\subsection{Conclusão}

Na primeira parte do capitulo foi apresentado um algoritmo via simulação para cálculo do prêmio de uma opção europeia. De posse do mesmo, foram propostas técnicas para melhoria da qualidade do resultado obtido. Em análise experimental, ficou comprovado a assertividade das técnicas apresentadas, com uma melhoria de mais de 4 vezes no erro padrão da simulação.

Após essa análise de precificação, estudamos o cálculo de risco de mercado do ponto de vista da métrica Value-at-Risk. Após conceituação do problema e de seus diferentes métodos de cálculo, apresentamos um algoritmo via Simulação de Monte Carlo proposto em GPU. Em análise experimental, o cálculo de VaR mostrou ser muito suscetível para GPGPU com um speedup de mais de 50 vezes.

\subsection{Notas e Leituras Complementares}

Para uma introdução simplificada ao cálculo de risco pelo VaR veja (Kimura et al., 2010). Para um aprofundamento no assunto, veja (Gregoriou, 2009), (Choudhry e Tanna, 2007) e (Jorion, 2003). Adicionalmente, (Cherubini e Lunga, 2007) fornece uma abordagem em gerenciamento de risco com foco em engenharia de software e em uma modelagem orientada a objetos.

(Hull, 2012) apresenta a precificação de outros tipos de opções, além dos vistos aqui, dentre outros produtos financeiros. Um guia completo de gestão de portfólios e precificação em finanças pode ser obtido em (Lee et al., 2010b). Para uma abordagem objetiva e simplificada do modelo de Black \& Scholes e apreçamento via não-arbitragem em língua portuguesa, veja (Zubelli e Souza, 2007). 


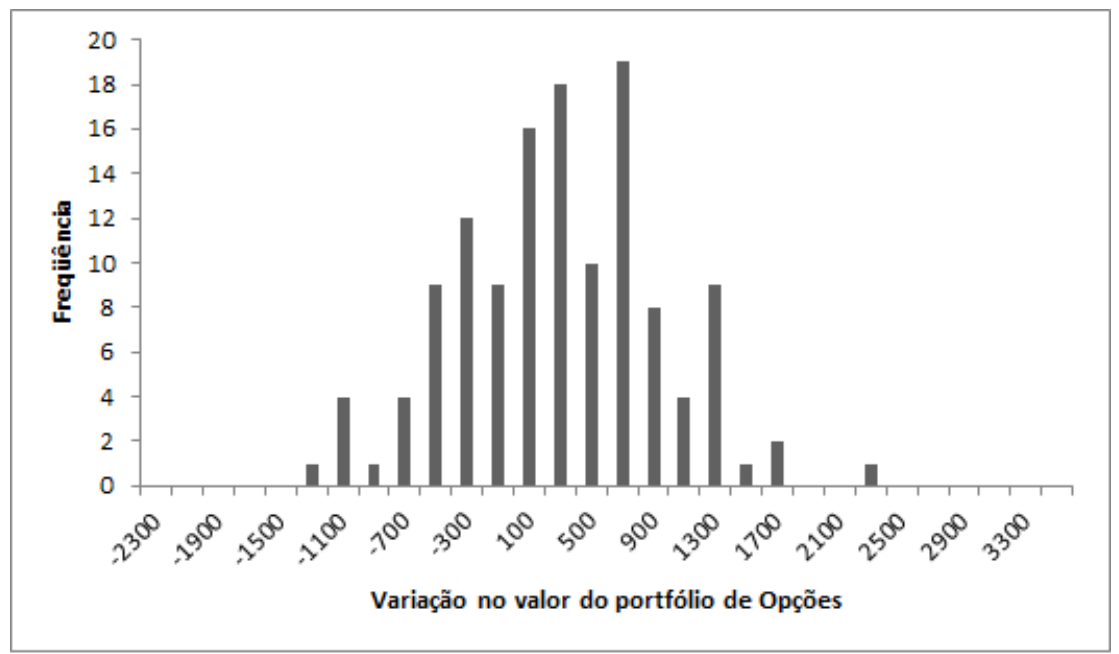

Figura 9.6: Variação no valor do Portfólio de Opções. Número de Simulações fixo em 128.

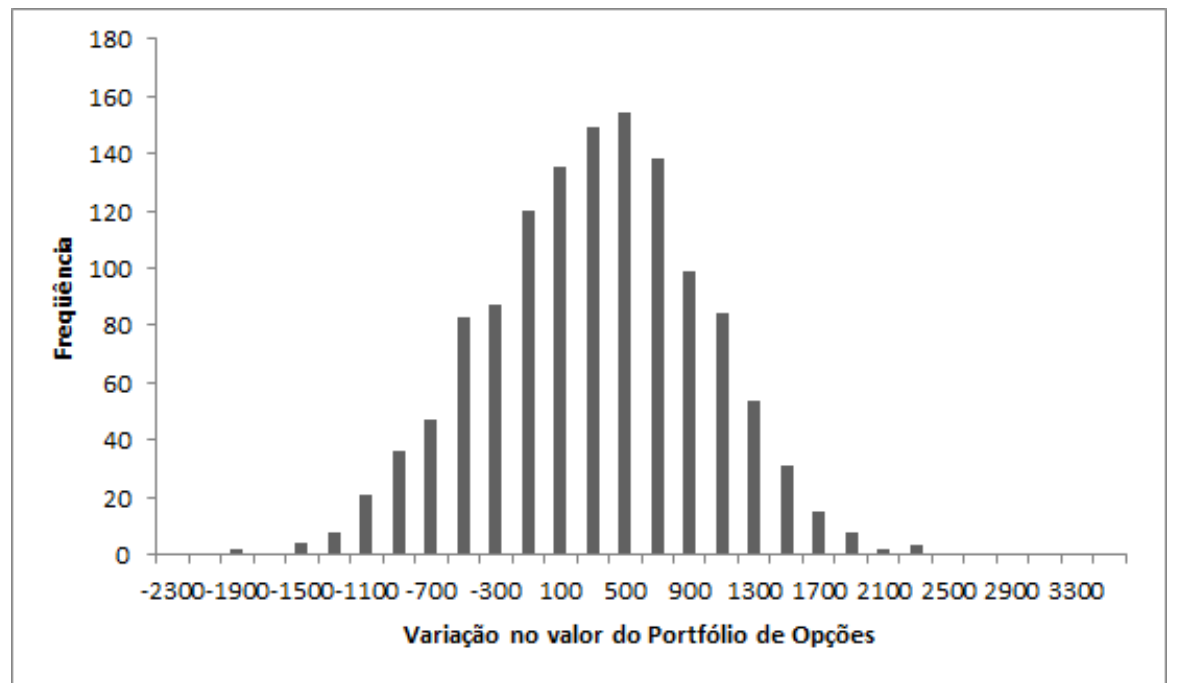

Figura 9.7: Variação no valor do Portfólio de Opções. Número de Simulações fixo em 1.280.

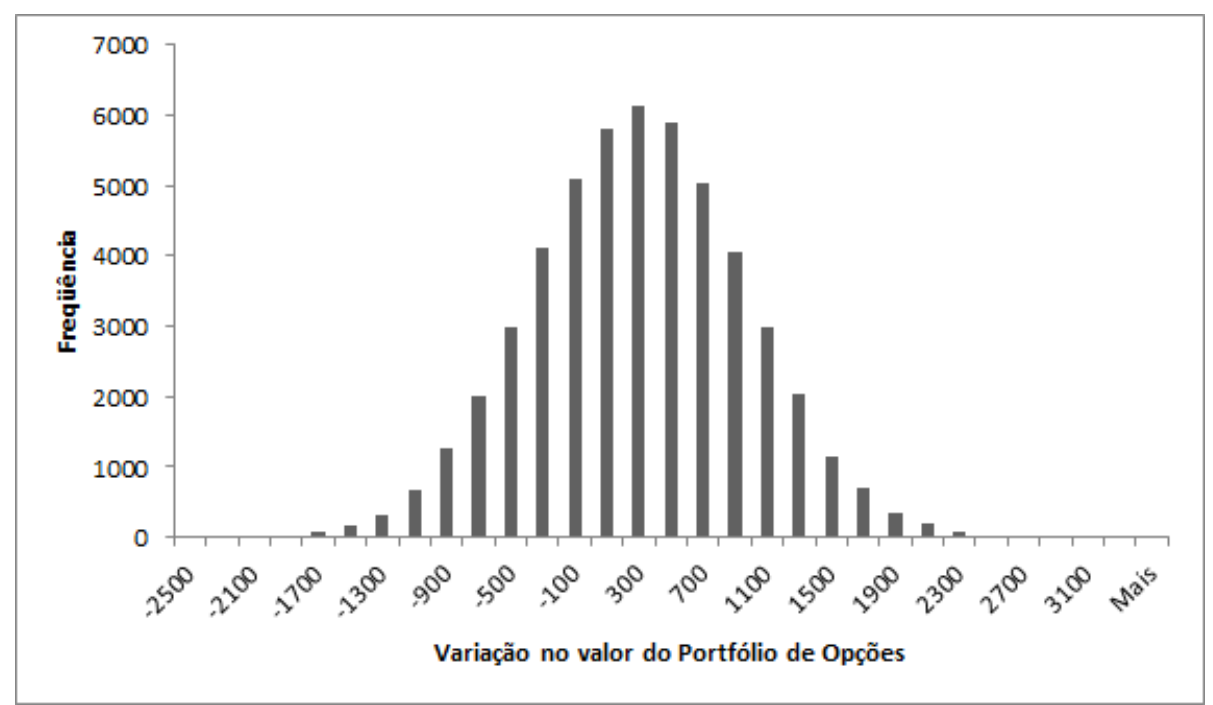

Figura 9.8: Variação no valor do Portfólio de Opções. Número de Simulações fixo em 51.200. 


\begin{tabular}{|c|c|c|}
\hline Número de Simulações & Tempo CPU (s) & Tempo GPU (s) \\
\hline \hline 1280 & 2,91 & 0,07 \\
\hline 3200 & 7,178 & 0,16 \\
\hline 6400 & 14,79 & 0,31 \\
\hline 12800 & 28,59 & 0,60 \\
\hline 25600 & 56,58 & 1,19 \\
\hline 38400 & 84,03 & 1,81 \\
\hline 51200 & 112,02 & 2,43 \\
\hline
\end{tabular}

Tabela 9.1: Tempo Computacional gasto em CPU e GPU no cálculo de VaR em função do número de simulações. Número de opções fixo em 2.000.

\begin{tabular}{|c|c|c|}
\hline Número de Opções & Tempo CPU (s) & Tempo GPU (s) \\
\hline \hline 500 & 28,03 & 0,99 \\
\hline 750 & 42,54 & 1,23 \\
\hline 1000 & 56,31 & 1,47 \\
\hline 1250 & 70,41 & 1,70 \\
\hline 1500 & 84,95 & 1,95 \\
\hline 1750 & 99,17 & 2,18 \\
\hline 2000 & 112,02 & 2,43 \\
\hline 2500 & 146,76 & 2,90 \\
\hline
\end{tabular}

Tabela 9.2: Tempo Computacional gasto em CPU e GPU no cálculo de VaR em função do número de opções no portfólio. Número de simulações fixo em 51200.

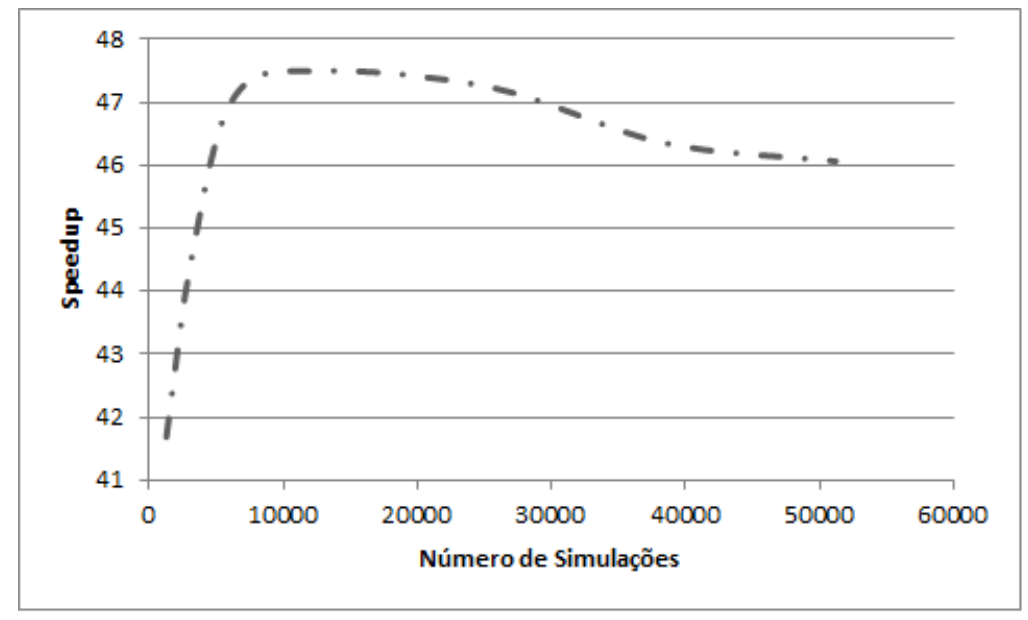

Figura 9.9: Speedup no cálculo de VaR de uma carteira com 2000 opções em função do número de simulações. 


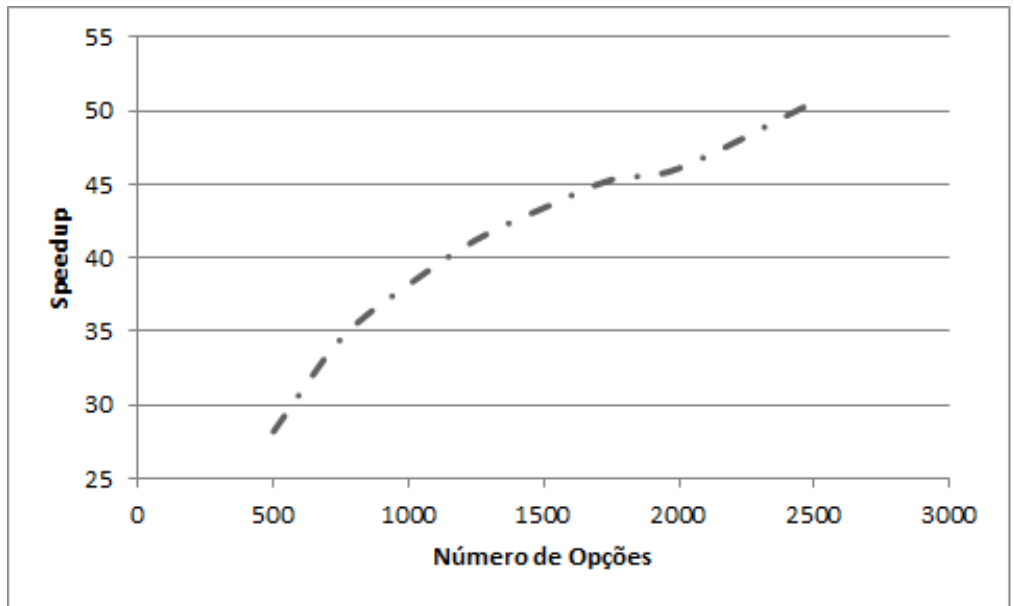

Figura 9.10: Speedup no cálculo de VaR em função do número de opções no portfólio. Número de simulações fixo em 51200. 


\section{Parte V}

\section{Conclusão}





\section{Capítulo 10}

\section{Conclusão}

Neste trabalho estudamos o ferramental matemático e computacional necessário para modelagem estocástica em finanças com a utilização de GPUs como plataforma de aceleração.

Para isso, apresentamos a GPU como uma plataforma de computação de propósito geral com a introdução a CUDA em uma arquitetura NVIDIA Fermi. Nesse sentido, apresentamos um resumo das principais técnicas de otimização utilizadas nessa plataforma.

Tendo em vista o papel base em simulações estocásticas de qualquer tipo, apresentamos um estudo dos mais diferentes tipos de PRNGs e QRNGs. Além de abordar técnicas de paralelização dos mesmos e estudar a portabilidade dos geradores Sobol e MRG32k3a em GPU. Em seguida, discutimos questões relativas a simulação de Monte Carlo e introduzimos a teoria de soluções de equações diferenciais estocásticas como base fundamental em modelagem estocástica em finanças.

Em um primeiro estudo de caso, resolvemos o problema de Stops Ótimos em finanças. Após definição formal do problema, revisamos o modelo trinomial proposto por (Warburtona e Zhang, 2006) e propomos um algoritmo alternativo para o mesmo via simulação utilizando uma técnica de aceitação e rejeição para probabilidade de transição dos preços de (Cox et al., 1979). O algoritmo sugerido é, comparativamente, de fácil implementação e naturalmente portável para GPU. Em seguida, apresentamos uma modelagem estocástica genérica para o problema e propomos um algoritmo para cálculo da esperança de ganho de um estratégia de Stop Gain. De posse desse algoritmo, resolvemos o problema de otimização de Stop Ótimo. Em análise experimental, as implementações em GPU mostraram ser mais escaláveis. Para o algoritmo de obtenção de esperança de retorno de um Stop Gain, foi alcançado um speedup de mais de 12 vezes.

No segundo estudo de caso, sobre cálculo de risco de mercado, inicialmente foi apresentado um algoritmo via simulação para precificação de opções. De posse do mesmo, foram propostas técnicas para melhoria da qualidade do resultado obtido. Em análise experimental, ficou comprovada a assertividade das técnicas apresentadas, com uma melhoria de mais de 4 vezes no erro padrão da simulação. Para o cálculo de risco pela métrica Value-at-Risk, com a proposição de um algoritmo via Simulação de Monte Carlo em GPU, foi obtido um speedup de mais de 50 vezes. 


\section{Parte VI}

Apêndice 



\section{Apêndice A}

\section{NVIDIA CUDA}

\section{A.1 Compute Capability}

\begin{tabular}{|c|c|c|c|c|c|}
\hline \multirow[b]{2}{*}{ Especificação Técnica } & \multicolumn{5}{|c|}{ Compute Capability } \\
\hline & 1.0 & 1.1 & 1.2 & 1.3 & 2.x \\
\hline $\begin{array}{l}\text { Máxima dimensão da grade de } \\
\text { blocos de threads }\end{array}$ & \multicolumn{4}{|c|}{2} & 3 \\
\hline $\begin{array}{l}\text { Máxima } x-, \text { y- ou z-dimensão da } \\
\text { grade de blocos }\end{array}$ & \multicolumn{5}{|c|}{65535} \\
\hline $\begin{array}{l}\text { Máxima dimensão de um bloco } \\
\text { de threads }\end{array}$ & \multicolumn{5}{|c|}{3} \\
\hline $\begin{array}{l}\text { Máxima x- ou y-dimensão de } \\
\text { blocos em eixos de threads }\end{array}$ & \multicolumn{4}{|c|}{512} & 1024 \\
\hline $\begin{array}{l}\text { Máxima z-dimensão de blocos } \\
\text { em eixos de threads }\end{array}$ & \multicolumn{5}{|c|}{64} \\
\hline Tamanho de um Warp & \multicolumn{5}{|c|}{32} \\
\hline $\begin{array}{l}\text { Máximo número de blocos } \\
\text { residentes por multiprocessador }\end{array}$ & \multicolumn{5}{|c|}{8} \\
\hline $\begin{array}{l}\text { Máximo número de warps } \\
\text { residentes por multiprocessador }\end{array}$ & & 24 & & 32 & 48 \\
\hline $\begin{array}{l}\text { Máximo número de threads } \\
\text { residentes por multiprocessador }\end{array}$ & & 768 & & 024 & 1536 \\
\hline $\begin{array}{l}\text { Número de registradores de } 32 \\
\text { bits por multiprocessador }\end{array}$ & & $8 k$ & & 16k & $32 \mathrm{k}$ \\
\hline $\begin{array}{l}\text { Máxima quantidade de Memória } \\
\text { Compartilhada por } \\
\text { multiprocessador }\end{array}$ & \multicolumn{4}{|c|}{$16 \mathrm{~KB}$} & $48 \mathrm{~KB}$ \\
\hline $\begin{array}{l}\text { Quantidade de memória local por } \\
\text { thread }\end{array}$ & \multicolumn{4}{|c|}{$16 \mathrm{~KB}$} & $512 \mathrm{~KB}$ \\
\hline Tamanho de Memória Constante & \multicolumn{5}{|c|}{$64 \mathrm{~KB}$} \\
\hline
\end{tabular}

Figura A.1: Especificação técnica por Compute Capability 
A.2 Funções Otimizadas pela Opção -use_fast_math

\begin{tabular}{|c|c|}
\hline Operação & Função \\
\hline $\mathrm{x} / \mathrm{y}$ & fdividef $(x, y)$ \\
\hline $\operatorname{sinf}(x)$ & $\operatorname{sinf}(x)$ \\
\hline $\cos f(x)$ & $\operatorname{cosf}(x)$ \\
\hline $\tan f(x)$ & $\operatorname{tanf}(\mathrm{x})$ \\
\hline $\sin \operatorname{cosf}(x, \mathrm{sptr}, \mathrm{cptr})$ & $\sin \operatorname{cosf}(x, s p t r, c p t r)$ \\
\hline $\log f(x)$ & $\log f(x)$ \\
\hline $\log 2 f(x)$ & $\log 2 f(x)$ \\
\hline $\log 10 f(x)$ & $\log 10 f(x)$ \\
\hline $\operatorname{expf}(x)$ & $\operatorname{expf}(\mathrm{x})$ \\
\hline $\exp 10 f(x)$ & $\exp 10 f(x)$ \\
\hline powf $(x, y)$ & powf $(x, y)$ \\
\hline
\end{tabular}

Figura A.2: Funções afetadas por-use_fast_math 


\section{Referências Bibliográficas}

Almasi e Gottlieb(1989) G. S. Almasi e A. Gottlieb. Highly Parallel Computing. Benjamin/Cummings, Redwood CA. Citado na pág. 9

Alves(2008) C.A.M. Alves. Modelos de volatilidade estocástica para o índice ibovespa: Reversão rápida à média e análise assintótica. Citado na pág. 60

Amdahl(1967) G. M. Amdahl. Validity of the single processor approach to achieving large scale computing capabilities. Em Proceedings of the April 18-20, 1967, spring joint computer conference, AFIPS '67 (Spring), páginas 483-485, New York, NY, USA. ACM. doi: 10.1145/1465482.1465560. URL http://doi.acm.org/10.1145/1465482.1465560. Citado na pág. xv, 13

Antonov e Saleev(1979) I. A. Antonov e V. M. Saleev. An economic method of computing lpt-sequences. Citado na pág. 44

Black e Scholes(1973) F. Black e M. Scholes. The pricing of options and corporate liabilities. Journal of Political Economy. Citado na pág. 55, 88

Box e Muller(1958) G. E. P. Box e M. E Muller. A note on the generation of random normal deviates. Ann. Math. Stat. Citado na pág. 40

Brodtkorb et al.(2012) A. R. Brodtkorb, T. R. Hagen e Ma. L. Saetra. Graphics processing unit (gpu) programming strategies and trends in gpu computing. Journal of Parallel and Distributed Computing. Citado na pág. xv, 17

Buluc et al.(2010) A. Buluc, J. R. Gilbert e C. Budak. Solving path problems on the gpu. Parallel Comput., 36(5-6):241-253. ISSN 0167-8191. doi: 10.1016/j.parco.2009.12.002. URL http://dx.doi.org/10.1016/j.parco.2009.12.002. Citado na pág. 3

C. Mazel e Hill(2011) B. Bachelet C. Mazel e D.R.C Hill. Shoverand: A model-driven framework to easily generate random numbers on gp-gpu. High Performance Computing and Simulation (HPCS). Citado na pág. xv, 45, 46

Che et al.(2008) S. Che, M. Boyer, J. Meng, D. Tarjan, J. W. Sheaffer e K. Skadron. A performance study of general-purpose applications on graphics processors using cuda. J. Parallel Distrib. Comput., 68(10):1370-1380. ISSN 0743-7315. doi: 10.1016/j.jpdc.2008.05.014. URL http://dx. doi.org/10.1016/j.jpdc.2008.05.014. Citado na pág. xv, 29, 30, 31

Cherubini e Lunga(2007) U. Cherubini e G. D. Lunga. Structured Finance: The Object Oriented Approach. The Wiley Finance Series. Wiley. ISBN 9780470512722. Citado na pág. 97

Choudhry e Tanna(2007) M. Choudhry e K. Tanna. An Introduction to Value-at-Risk. Securities Institute. Wiley. ISBN 9780470033777. Citado na pág. 92, 97

Chow et al.(1971) Y.S. Chow, H. Robbins e D. Siegmund. Great expectations: the theory of optimal stopping. Houghton Mifflin. Citado na pág. 86 
Cover(1987) T. M. Cover. Gambler's ruin: A random walk on the simplex. Open Problems in Communications and Computation. Citado na pág. 76

Cox et al.(1979) J. Cox, S. Ross e M. Rubenstein. Option pricing: a simplified approach. Journal of Financial Economics. Citado na pág. ix, xvi, 75, 76, 103

Dally e Nickolls(2010) W. J. Dally e J. Nickolls. The gpu computing era. IEEE Computer Society. Citado na pág. xv, 17, 18, 19

Dehne e Yogaratnam(2010) F. Dehne e K. Yogaratnam. Exploring the limits of gpus with parallel graph algorithms. CoRR, abs/1002.4482. Citado na pág. 3

Dixon et al.(2009) M. Dixon, J. Chong e K. Keutzer. Acceleration of market value-at-risk estimation. Em Proceedings of the 2nd Workshop on High Performance Computational Finance, WHPCF '09, páginas 5:1-5:8, New York, NY, USA. ACM. ISBN 978-1-60558-716-5. doi: 10.1145/1645413.1645418. URL http://doi.acm.org/10.1145/1645413.1645418. Citado na pág. 3, 93

Egloff(2010) D. Egloff. High performance finite difference PDE solvers on GPUs, 2010. Citado na pág. 3

Fernando(2003) M. J. Fernando, R. e Kilgard. The Cg Tutorial: The Definitive Guide to Programmable Real-Time Graphics. Addison-Wesley Professional. Citado na pág. 16

Fischer et al.(1999) G. W. Fischer, Z. Carmon, D. Ariely, G. Zauberman e P. L'Ecuyer. Good parameters and implementations for combined multiple recursive random number generators. Oper. Res., 47(1):159-164. ISSN 0030-364X. doi: 10.1287/opre.47.1.159. URL http://dx.doi.org/ 10.1287/opre.47.1.159. Citado na pág. 42

Flynn(1972) M. J. Flynn. Some Computer Organizations and Their Effectiveness. IEEE Trans. on Computing. Citado na pág. 9

Giles et al.(2008) M. B. Giles, F. Y. Kuo, I. H. Sloan e B. J. Waterhouse. Quasi-monte carlo for finance applications. Em Geoffry N. Mercer e A. J. Roberts, editors, Proceedings of the 14th Biennial Computational Techniques and Applications Conference, CTAC-2008, volume 50 of ANZIAM J., páginas C308-C323. Citado na pág. 95

Glasserman(2004) P. Glasserman. Monte Carlo Methods in Financial Engineering. Applications of Mathematics Series. Springer. ISBN 9780387004518. Citado na pág. 76

Govindaraju e Manocha(2007) N. K. Govindaraju e D. Manocha. Cache-efficient numerical algorithms using graphics hardware. Parallel Comput., 33(10-11):663-684. ISSN 0167-8191. doi: 10.1016/j.parco.2007.09.006. URL http://dx.doi.org/10.1016/j.parco.2007.09.006. Citado na pág. 20

Gregoriou(2009) G. N. Gregoriou. The VAR Implementation Handbook. McGraw-Hill Finance \& Investing. McGraw-Hill Companies,Incorporated. ISBN 9780071615136. Citado na pág. 97

Gut(2009) A. Gut. Stopped Random Walks: Limit Theorems and Applications. Springer series in operations research. Springer. ISBN 9780387878355. Citado na pág. 86

Harish e Narayanan(2007) P. Harish e P. J. Narayanan. Accelerating large graph algorithms on the gpu using cuda. Em Proceedings of the 14th international conference on High performance computing, HiPC'07, páginas 197-208, Berlin, Heidelberg. Springer-Verlag. ISBN 3-540-77219-7, 978-3-540-77219-4. URL http://dl.acm.org/citation.cfm?id=1782174.1782200. Citado na pág. 3

Hida(1980) T. Hida. Brownian Motion. Springer. Citado na pág. 57 
Hong e Kim(2009) S. Hong e H. Kim. An analytical model for a gpu architecture with memorylevel and thread-level parallelism awareness. Em Proceedings of the 36th annual international symposium on Computer architecture, ISCA '09, páginas 152-163. ACM. ISBN 978-1-60558-5260. doi: 10.1145/1555754.1555775. Citado na pág. 31

Hull(2012) J. Hull. Options, Futures, and Other Derivatives and DerivaGem CD Package. Prentice Hall. ISBN 9780132777421. Citado na pág. xvi, 71, 75, 88, 91, 97

Huynh et al.(2011) H. T. Huynh, V. S. Lai e I. Soumare. Stochastic Simulation and Applications in Finance with MATLAB Programs. The Wiley Finance Series. Wiley. ISBN 9780470722138. Citado na pág. xv, 39, 74, 75

IEEE Task P754(1985) IEEE Task P754. ANSI/IEEE 754-1985, Standard for Binary FloatingPoint Arithmetic. IEEE, New York, Agosto 12 1985. Citado na pág. 18

IEEE Task P754(2008) IEEE Task P754. IEEE 754-2008, Standard for Floating-Point Arithmetic. Citado na pág. 18

Imkeller e Rogers(2010) N. Imkeller e L. C. G. Rogers. Trading to stops. URL http://www. statslab.cam.ac.uk/ chris/papers.html. Citado na pág. 71

Jasra e Del Moral(2011) Ajay Jasra e Pierre Del Moral. Sequential monte carlo methods for option pricing. doi: 10.1080/07362994.2011.548993. Citado na pág. 90

Joe e Kuo(2008) S. Joe e F. Y. Kuo. Código fonte: Sobol sequence generator, 2008. URL http://web.maths.unsw.edu.au/ fkuo/sobol/. Último acesso em 29/01/2013. Citado na pág. 68

Jorion(2003) P. Jorion. Value at risk: a nova fonte de referência para a gestão do risco financeiro. Bolsa de Mercadorias \& Futuros. ISBN 9788574380070. Citado na pág. xvi, 93, 97

Jung e O'Leary(2006) J. H. Jung e D. P. O'Leary. Cholesky decomposition and linear programming on a gpu. Dissertação de Mestrado, University of Maryland. Citado na pág. 95

Jung e O'Leary(2009) J. H. Jung e D. P. O'Leary. Implementing an interior point method for linear programs on a CPU-GPU system. Electronic Transactions on Numerical Analysis. Citado na pág. 3

Kaplan(1991) W. Kaplan. Advanced Calculus. Addison-Wesley, Advanced Book Program. ISBN 9780201578881. Citado na pág. 58

Karlin e Taylor(1975) S. Karlin e H. M. Taylor. A First Course in Stochastic Processes. Number vol. 1. Academic Press. ISBN 9780123985521. Citado na pág. 86

Kimura et al.(2010) H. Kimura, A. S. Suen, L. C. J. Perera e L. F. C. Basso. Value at Risk Como Entender e Calcular o Risco pelo VaR. INSIDE BOOKS. ISBN 9788560550074. Citado na pág. 87, 92, 97

Kirk e Hwu(2010) D. B. Kirk e W. W. Hwu. Programming Massively Parallel Processors: A Hands-on Approach. Applications of GPU Computing Series. Elsevier Science. ISBN 9780123814739. Citado na pág. xv, 15, 16

Knuth(1997) D. E. Knuth. The art of computer programming, volume 2 (3rd ed.): seminumerical algorithms. Addison-Wesley Longman Publishing Co., Inc., Boston, MA, USA. ISBN 0-20189684-2. Citado na pág. 35, 36, 42

L'Ecuyer(2006) P. L'Ecuyer. Testu01: A c library for empirical testing of random number generators. ACM Transactions on Mathematical Software. Citado na pág. 36 
L'Ecuyer(2007) P. L'Ecuyer. Random Number Generation, páginas 93-137. John Wiley \& Sons, Inc. ISBN 9780470172445. doi: 10.1002/9780470172445.ch4. URL http://dx.doi.org/10.1002/ 9780470172445.ch4. Citado na pág. 35

Lee et al.(2010a) A. Lee, C. Yau, M. B. Giles, A. Doucet e C. C. Holmes. On the utility of graphics cards to perform massively parallel simulation of advanced monte carlo methods. Journal of Computational and Graphical Statistics, páginas 769-789. Citado na pág. 3

Lee et al.(2010b) C. F. Lee, A. C. Lee e J. Lee. Handbook of Quantitative Finance and Risk Management. Springer. ISBN 9780387771175. Citado na pág. 97

Lee et al.(2009) M. Lee, C. H. Chun e S. Hong. Financial derivatives modeling using gpu's. Em Proceedings of the 2009 International Conference on Scalable Computing and Communications; Eighth International Conference on Embedded Computing, SCALCOM-EMBEDDEDCOM '09, páginas 440-445. IEEE Computer Society. ISBN 978-0-7695-3825-9. doi: 10.1109/ EmbeddedCom-ScalCom.2009.85. Citado na pág. 40

Marsaglia(1995) G. Marsaglia. The diehard battery of tests of randomness. Citado na pág. 36

Marsaglia(2003) G. Marsaglia. Xorshift rngs. Journal of Statistical Software, 8(14):1-6. ISSN 1548-7660. URL http://www.jstatsoft.org/v08/i14. Citado na pág. 45

Marsaglia e Tsang(2000) G. Marsaglia e W. W. Tsang. The ziggurat method for generating random variables. Journal of Statistical Software, 5(8):1-7. Citado na pág. 40

Matsumoto e Nishimura(1998) M. Matsumoto e T. Nishimura. Mersenne twister: A 623dimensionally equidistributed uniform pseudorandom number generator. ACM Transactions on Modeling and Computer Simulation. Citado na pág. 37

Matsumoto e Nishimura(2009) M. Matsumoto e T. Nishimura. A c-program for mt19937, 2009. URL http://www.math.sci.hiroshima-u.ac.jp/ $\sim \mathrm{m}-\mathrm{mat} / \mathrm{MT} / \mathrm{emt} . h t m l$. Último acesso em 29/01/2013. Citado na pág. 68

Matteis e Pagnutti(1990) A. De Matteis e S. Pagnutti. Long-range correlations in linear and non-linear random number generators. Parallel Computing. Citado na pág. 41

Mattson et al.(2004) T. G. Mattson, B. A. Sanders e B. L. Massingil. Patterns for Parallel Programming. Addison-Wesley Professional. Citado na pág. xv, 9, 10, 11

Mikosch(1999) T. Mikosch. Elementary Stochastic Calculus With Finance in View. World Scientific Publishing Company. Citado na pág. 63

Miller et al.(2010) F. P. Miller, A. F. Vandome e M. B. John. Marsaglia Polar Method. VDM Publishing. ISBN 9786132746498. Citado na pág. 40

Muller e Frauendiener(2013) T. Muller e J. Frauendiener. Charged particles constrained to a curved surface. European Journal of Physics, 34(1):147. URL http://stacks.iop.org/0143-0807/ $34 / \mathrm{i}=1 / \mathrm{a}=147$. Citado na pág. 3

Narayana et al.(2012) D. Narayana, P. Somawanshi e M. Joshi. Stochastic differential equations simulation using gpu. Citado na pág. 62

Nguyen(2007) H. Nguyen. Gpu gems 3. Addison-Wesley Professional. ISBN 9780321545428. Citado na pág. 35

NVIDIA(2009a) NVIDIA. White paper NVIDIA's Next Generation CUDA Compute Architecture: Fermi. Citado na pág. xv, 19, 20 
NVIDIA(2009b) NVIDIA. Site de computação em finanças da nvidia, 2009b. URL http://www. nvidia.com/object/computational_finance.html. Último acesso em 29/01/2013. Citado na pág. xv, 4

NVIDIA(2010a) NVIDIA. Cuda Ocuppancy Calculator. URL http://developer.download.nvidia. com/compute/cuda/3_1/sdk/docs/CUDA_Occupancy_calculator.xls. Citado na pág. 31

NVIDIA(2010b) NVIDIA. Cuda C Best Practices Guide 3.2. Citado na pág. 29

NVIDIA(2011) NVIDIA. Cuda 4.0 math libraries performance. NVDIA Corporation. Citado na pág. 45

NVIDIA(2011a) NVIDIA. Cuda C Programming Guide 4.0. Citado na pág. xv, 21, 22, 29, 32

Oksendal(1992) B. Oksendal. Stochastic Differential Equations. Springer-Verlag. Citado na pág. 72, 86

Pages e Wilbertz(2010) G. Pages e B. Wilbertz. Parallel implementation of quantization methods for the valuation of swing options on gpgpu. Em High Performance Computational Finance (WHPCF), 2010 IEEE Workshop on, páginas 1 -5. doi: 10.1109/WHPCF.2010.5671811. Citado na pág. 3,91

Pages e Wilbertz(2012) G. Pages e B. Wilbertz. Gpgpus in computational finance: massive parallel computing for american style options. Concurr. Comput. : Pract. Exper., 24(8):837-848. ISSN 1532-0626. doi: 10.1002/cpe.1774. Citado na pág. 3, 91

Preis(2011) T. Preis. GPU-computing in econophysics and statistical physics. The European Physical Journal - Special Topics, 194(1):87-119. ISSN 1951-6355. doi: 10.1140/epjst/e2011-01398-x. URL http://dx.doi.org/10.1140/epjst/e2011-01398-x. Citado na pág. 3

Reuillon et al.(2008) R. Reuillon, D. Hill, Z. El Bitar e V. Breton. Rigorous distribution of stochastic simulations using the distme toolkit. IEEE Transactions On Nuclear Science. Citado na pág. 41

Roy(2002) R. Roy. Comparison of different techniques to generate normal random variables. Citado na pág. 40

Sadiq et al.(2012) S. K. Sadiq, F. Noé e G. De Fabritiis. Kinetic characterization of the critical step in hiv-1 protease maturation. Proceedings of the National Academy of Sciences. doi: 10. 1073/pnas.1210983109. Citado na pág. 3

Saito et al.(2012) K. Saito, E. Koizumi e H. Koizumi. Application of parallel hybrid algorithm in massively parallel gpgpu-the improved effective and efficient method for calculating coulombic interactions in simulations of many ions with simion. Journal of The American Society for Mass Spectrometry, 23:1609-1615. ISSN 1044-0305. doi: 10.1007/s13361-012-0435-6. URL http: //dx.doi.org/10.1007/s13361-012-0435-6. Citado na pág. 3

Satish et al.(2009) Na. Satish, M. Harris e M. Garland. Designing efficient sorting algorithms for manycore gpus. Em Proceedings of the 2009 IEEE International Symposium on ParallelE3Distributed Processing, IPDPS '09, páginas 1-10, Washington, DC, USA. IEEE Computer Society. ISBN 978-1-4244-3751-1. doi: 10.1109/IPDPS.2009.5161005. URL http://dx.doi.org/10. 1109/IPDPS.2009.5161005. Citado na pág. 3

Shen e Wang(2001) S. Shen e A. Wang. On stop-loss strategies for stock investments. Applied Mathematics and Computation. Citado na pág. 71

Shiryaev e Aries(2008) A. N. Shiryaev e A. B. Aries. Optimal Stopping Rules. Applications of mathematics. Springer-Verlag Berlin Heidelberg. ISBN 9783540740117. Citado na pág. 86 
Sobol(1967) I. M. Sobol. On the distribution of points in a cube and the approximate evaluation of integrals. Computational Mathematics and Mathematical Physics, 7(4):86+. Citado na pág. 43

Solomon et al.(2010) S. Solomon, R. K. Thulasiram e P. Thulasiraman. Option pricing on the gpu. Em Proceedings of the 2010 IEEE 12th International Conference on High Performance Computing and Communications, HPCC '10, páginas 289-296, Washington, DC, USA. IEEE Computer Society. ISBN 978-0-7695-4214-0. doi: 10.1109/HPCC.2010.54. Citado na pág. 3, 91

Spampinato e Elstery(2009) D. G. Spampinato e A. C. Elstery. Linear optimization on modern gpus. Em Proceedings of the 2009 IEEE International Symposium on ParallelEDDistributed Processing, IPDPS '09, páginas 1-8, Washington, DC, USA. IEEE Computer Society. ISBN 9781-4244-3751-1. doi: 10.1109/IPDPS.2009.5161106. URL http://dx.doi.org/10.1109/IPDPS.2009. 5161106. Citado na pág. 3

Stojanovski et al.(2012) M. Stojanovski, D. Gjorgjevikj e G. Madjarov. Parallelization of dynamic programming in nussinov rna folding algorithm on the cuda gpu. Em Ljupco Kocarev, editor, $I C T$ Innovations 2011, volume 150 of Advances in Intelligent and Soft Computing, páginas 279-289. Springer Berlin Heidelberg. ISBN 978-3-642-28663-6. Citado na pág. 3

W. H. Press e Flannery(2007) W. T. Vetterling W. H. Press, S. A. Teukolsky e B. P. Flannery. Numerical Recipes 3rd Edition: The Art of Scientific Computing. Cambridge University Press, New York, NY, USA, 3 edição. ISBN 0521880688, 9780521880688. Citado na pág. 80

Wald(1945) A. Wald. Sequential tests of statistical hypotheses. The Annals of Mathematical Statistics, 16(2):117-186. ISSN 00034851. doi: 10.2307/2235829. Citado na pág. 86

Warburtona e Zhang(2006) A. Warburtona e Z. G. Zhang. A simple computational model for analyzing the properties of stop-loss, take-profit, and price breakout trading strategies. Computers and Operations Research. Citado na pág. ix, 71, 72, 73, 75, 81, 86, 103

Zhang(2001) Q. Zhang. Stock trading: an optimal selling rule. SIAM Journal on Control and Optimization. Citado na pág. 71,75

Zubelli e Souza(2007) J. Zubelli e M. O. Souza. Modelagem Matemática em Finanças Quantitativas em Tempo Discreto. Notas em Matemática Aplicada. SBMAC. ISBN 9788586883347. Citado na pág. 97 


\section{Índice Remissivo}

Arquitetura

de memória distribuída, 11

de Von Neumann, 9

Fermi, 18

ECC, 20

SFU, 18

MIMD, 10

MISD, 9

NUMA, 11

SIMD, 9

SISD, 9

SMP, 10

Central Server, 41

CUDA, 21

CURAND, 45

Discretização

de Euler, 62

de Milstein, 62

de Euler, 79

Distribuição

de probabilidade de transição, 74

de probabilidade terminal, 74

Gaussiana, 40, 74

não uniforme, 39

Equações Diferenciais Estocásticas, 58

Soluções numéricas de, 60

GPGPU, 16

GPU, 15

\section{IEEE}

754-1985, 18

754-2008, 18

Instrução

FMA, 18

$\mathrm{LD} / \mathrm{ST}, 18$

MAD, 18

Itô

Integral de, 58

Lema de, 59

Processo de, 58
Lagged Fibonacci Generator, 36

Leap Frog, 41

Linear Congruential Generator, 36

Método

de Aceitação e Rejeição, 40, 76

de Box-Muller, 40

de Brent, 80

de Euler, 61

de Inversão, 40

de Monte Carlo, 49

de Runge-Kutta, 61

de Ziggurat, 40

Implícito, 61

Polar, 40

Memória

Coalesced, 23

Global, 23

Local, 23

Mersenne Twister, 37, 95

Modelo

binomial de preços, 75

de Black\&Scholes, 88

de precificação, 75

estocástico de Stop, 77

trinomial de preços, 73

trinomial estocástico, 76

Movimento Browniano, 55

Geométrico, 55

representado como uma série, 57

Simulação do, 56

Multiple Recursive Generator, 36 em GPU, 42

NVIDIA

GeForce

3,16

256,16

6800,16

8800,16

FX, 16

GT 525M, 67

RIVA 128, 16 
Tesla

C870, 17

D870, 17

S870, 17

Ocupação, 31, 68

Opção

Americana, 87

com Barreiras, 71

de Call, 87

de Put, 87

Europeia, 87

Payoff de, 90

Precificação de, 87

Passeio Aleatório, 53

dos preços, 73

PRNG, 35, 36

Processo

de Itô, 58

de Ornstein-Uhlenbeck, 60

de Wiener, 74

Estocástico, 54

Log-Normal, 78

QRNG, 35, 37

Random Spacing, 41

Registradores, 23

Representação de

Lévy-Ciesielski, 57

Paley-Wiener, 57

RNG, 35

Ruína do apostador, 76

Série de Fourier, 57

Sequência

de Faure, 39

de Halton, 38

de Van Der Corput, 37

Sequence Splitting, 41

ShoveRand, 45

Skip-ahead, 41

Sobol, 43, 95

em GPU, 44

Stop, 71

Gain, 71

Loss, 71

Móvel, 76

Técnica de amostragem estratificada, 52 análise de componentes principais, 95 variáveis antitéticas, 50, 91 variável de controle, 52, 91

Taxa de juros livre de risco, 75

Taxionomia de Flynn, 9

Tempo de Parada, 72

Thrust

random, 45

TRNG, 35

Value-at-Risk, 92

VaR

Modelo

de Variâncias-Covariâncias, 93

Delta-Normal de, 93

Paramétrico de, 93

Simulação

de Monte Carlo de, 94

Histórica de, 93 